University of Tennessee Health Science Center

UTHSC Digital Commons

\title{
Predictors of Depressive and Anxiety Symptoms among African American HIV-positive Women
}

Nina Katherine Sublette

University of Tennessee Health Science Center

Follow this and additional works at: https://dc.uthsc.edu/dissertations

Part of the Immune System Diseases Commons, Psychiatric and Mental Health Commons, Psychiatric and Mental Health Nursing Commons, Psychological Phenomena and Processes Commons, Virus Diseases Commons, and the Women's Health Commons

\section{Recommended Citation}

Sublette, Nina Katherine, "Predictors of Depressive and Anxiety Symptoms among African American HIVpositive Women" (2008). Theses and Dissertations (ETD). Paper 266. http://dx.doi.org/10.21007/ etd.cghs.2008.0303.

This Dissertation is brought to you for free and open access by the College of Graduate Health Sciences at UTHSC Digital Commons. It has been accepted for inclusion in Theses and Dissertations (ETD) by an authorized administrator of UTHSC Digital Commons. For more information, please contact jwelch30@uthsc.edu. 


\title{
Predictors of Depressive and Anxiety Symptoms among African American HIV- positive Women
}

\begin{abstract}
This descriptive correlation study had four purposes: describe depressive and anxiety symptom levels in pregnant and non-pregnant African American (AA) HIV-positive women, examine the association between underlying vulnerabilities and depressive symptoms in HIV-positive AA women, determine the association between underlying vulnerabilities and anxiety symptoms in AA HIV-positive women, and determine the predictors of depressive and anxiety symptoms among African American HIV-positive women. A convenience sample of 80 African American HIV-positive women was recruited from university-based obstetrics/-gynecology and infectious disease practices in a large metropolitan city in the Mid-South. Depressive symptoms were measured with the Center for Epidemiologic Studies Depression Scale (CESD) and Edinburgh Postnatal depression Scale (EPDS). Anxiety symptoms were measured with the Beck Anxiety Inventory (BAI). Sleep quality was measured with the Pittsburg Sleep Quality Index (PSQI). An investigator-developed demographic data form was used to obtain patient characteristics including data about inherited and acquired vulnerabilities. Inherited vulnerabilities included family history of mood disorders and family history of alcohol and/or drug use. Acquired vulnerabilities included educational level, income level, alcohol and/or use, HIV disclosure status, sleep quality, and history of sexual assault. The stress diathesis theory provided the conceptual basis for the study. The sample consisted of both pregnant $(n=20)$ and non-pregnant $(n=60)$ women. Participants were typically single $(57.5 \%)$, middleaged (33.21 \pm 10.7 years), and had completed high school (30\%) or some college (35\%). Poverty was prevalent in the sample, with $70.5 \%$ reporting annual household incomes below $\$ 10,000$. Most women (92.5\%) had disclosed their HIV status. Almost half of the sample (42\%) reported a history of sexual assault. Substance abuse was higher in the non-pregnant group of women (40\%) than the non-pregnant group (5\%) of women. Median depressive symptom scores for the total sample were mildly elevated. Median anxiety symptom scores for the total sample illustrated little or no anxiety symptoms. Poor sleep quality was prevalent in both groups and was associated with elevated CES-D, EPDS, and BAI scores in both groups of women. Family history of mood disorders was associated with elevated CES-D scores in the group of pregnant women, and elevated BAI scores in both groups. HIV disclosure was associated with elevated CES-D scores in the pregnant group, and substance use with elevated CES-D scores, in the non-pregnant group. Non-pregnant HIV-positive women had higher depressive symptom scores on both the CES-D and EPDS when compared to pregnant HIV-positive women. Regression analyses were performed to determine which independent variables predicted elevated depressive and anxiety symptom scores. Substance use and poor sleep quality were statistically significant predictors, accounting for $55 \%$ of the variance in CES-D scores, $43 \%$ of the variance in EPDS scores, and $53 \%$ of the variance in BAI scores. Future studies should include testing interventions that improve sleep quality. Untreated mood disorders are problematic in HIV-positive patients, as depression is associated with decreased adherence to highly active antiretroviral medications. Ongoing assessments of depressive and anxiety symptoms are necessary, as clinically significant levels resulting in referrals were warranted.
\end{abstract}

\section{Document Type}

Dissertation

\section{Degree Name}

Doctor of Philosophy (PhD)

\section{Program}

Nursing 


\section{Research Advisor}

Mona Newsome Wicks, Ph.D.

\section{Keywords}

Anxiety, depression, African American, HIV-positive women, inherited vulnerabilities, acquired vulnerabilities

\section{Subject Categories}

Diseases | Immune System Diseases | Medicine and Health Sciences | Nursing | Psychiatric and Mental Health | Psychiatric and Mental Health Nursing | Psychological Phenomena and Processes | Virus Diseases | Women's Health 


\title{
PREDICTORS OF DEPRESSIVE AND ANXIETY SYMPTOMS AMONG AFRICAN AMERICAN HIV-POSITIVE WOMEN
}

\author{
A Dissertation \\ Presented for \\ The Graduate Studies Council \\ The University of Tennessee \\ Health Science Center
}

\author{
In Partial Fulfillment \\ Of the Requirements for the Degree \\ Doctor of Philosophy \\ From The University of Tennessee
}

By

Nina Katherine Sublette

May 2008 
Copyright (C) 2008 by Nina Katherine Sublette All rights reserved 


\section{ACKNOWLEDGEMENTS}

I would like to thank the members of my dissertation committee for their dedication and support during my long career as a doctoral student. The completion of this dissertation would not have been accomplished without the unending encouragement from my advisor, Mona N. Wicks, PhD.

I would also like to thank my colleagues, Edwin M. Thorpe, Jr, MD and Patricia M. Flynn, MD for their support, encouragement, and continuous education. I appreciate your dedication to me, HIV-positive women, and the unending quest to prevent mother to child transmission of HIV.

Lastly, I would like to thank the women that participated in this study. Without your participation and willingness to address sensitive issues, this project could not have been completed. 


\begin{abstract}
This descriptive correlation study had four purposes: describe depressive and anxiety symptom levels in pregnant and non-pregnant African American (AA) HIVpositive women, examine the association between underlying vulnerabilities and depressive symptoms in HIV-positive AA women, determine the association between underlying vulnerabilities and anxiety symptoms in AA HIV-positive women, and determine the predictors of depressive and anxiety symptoms among African American HIV-positive women. A convenience sample of 80 African American HIV-positive women was recruited from university-based obstetrics/-gynecology and infectious disease practices in a large metropolitan city in the Mid-South. Depressive symptoms were measured with the Center for Epidemiologic Studies Depression Scale (CES-D) and Edinburgh Postnatal depression Scale (EPDS). Anxiety symptoms were measured with the Beck Anxiety Inventory (BAI). Sleep quality was measured with the Pittsburg Sleep Quality Index (PSQI). An investigator-developed demographic data form was used to obtain patient characteristics including data about inherited and acquired vulnerabilities. Inherited vulnerabilities included family history of mood disorders and family history of alcohol and/or drug use. Acquired vulnerabilities included educational level, income level, alcohol and/or use, HIV disclosure status, sleep quality, and history of sexual assault. The stress diathesis theory provided the conceptual basis for the study. The sample consisted of both pregnant $(n=20)$ and non-pregnant $(n=60)$ women. Participants were typically single (57.5\%), middle-aged (33.21 \pm 10.7 years), and had completed high school $(30 \%)$ or some college $(35 \%)$. Poverty was prevalent in the sample, with $70.5 \%$ reporting annual household incomes below $\$ 10,000$. Most women $(92.5 \%)$ had disclosed their HIV status. Almost half of the sample (42\%) reported a history of sexual assault. Substance abuse was higher in the non-pregnant group of women (40\%) than the non-pregnant group (5\%) of women. Median depressive symptom scores for the total sample were mildly elevated. Median anxiety symptom scores for the total sample illustrated little or no anxiety symptoms. Poor sleep quality was prevalent in both groups and was associated with elevated CES-D, EPDS, and BAI scores in both groups of women. Family history of mood disorders was associated with elevated CES-D scores in the group of pregnant women, and elevated BAI scores in both groups. HIV disclosure was associated with elevated CES-D scores in the pregnant group, and substance use with elevated CES-D scores, in the nonpregnant group. Non-pregnant HIV-positive women had higher depressive symptom scores on both the CES-D and EPDS when compared to pregnant HIV-positive women. Regression analyses were performed to determine which independent variables predicted elevated depressive and anxiety symptom scores. Substance use and poor sleep quality were statistically significant predictors, accounting for $55 \%$ of the variance in CES-D scores, $43 \%$ of the variance in EPDS scores, and $53 \%$ of the variance in BAI scores. Future studies should include testing interventions that improve sleep quality. Untreated mood disorders are problematic in HIV-positive patients, as depression is associated with decreased adherence to highly active antiretroviral medications. Ongoing assessments of depressive and anxiety symptoms are necessary, as clinically significant levels resulting in referrals were warranted.
\end{abstract}




\section{TABLE OF CONTENTS}

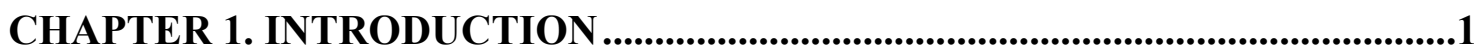

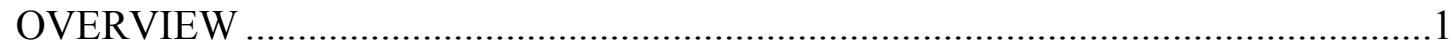

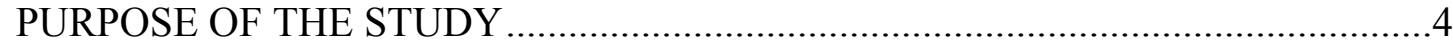

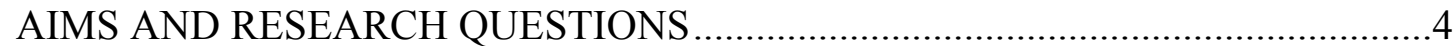

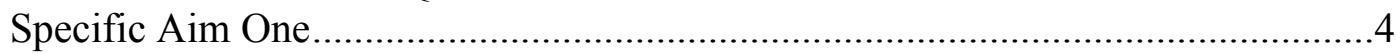

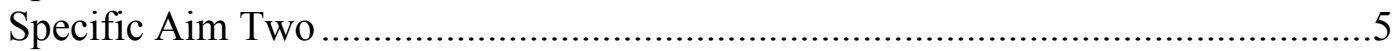

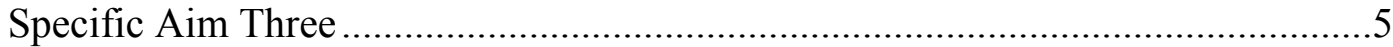

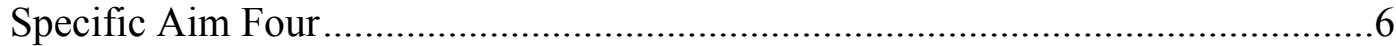

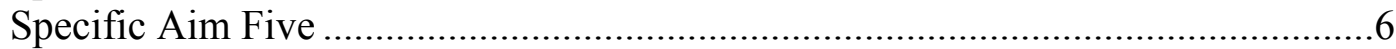

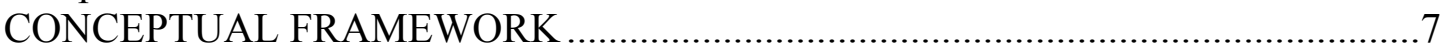

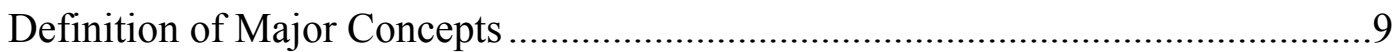

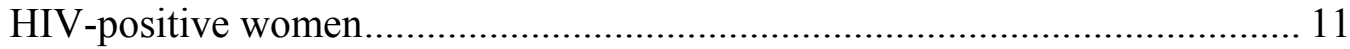

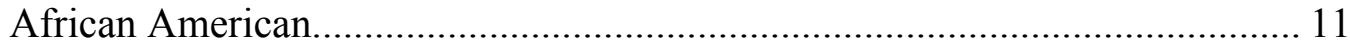

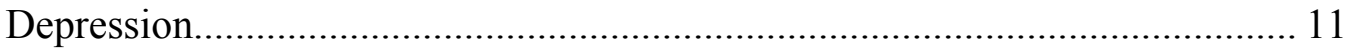

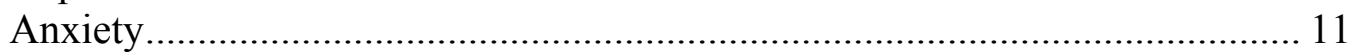

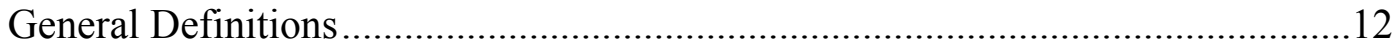

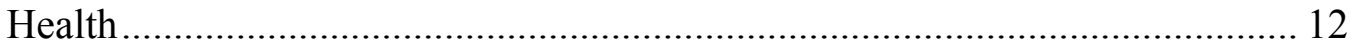

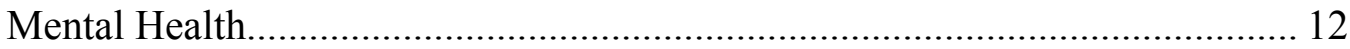

Inherited Vulnerabilities ......................................................................... 12

Acquired Vulnerabilities .......................................................................... 12

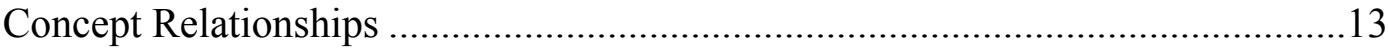

SIGNIFICANCE OF STUDY …………..................................................14

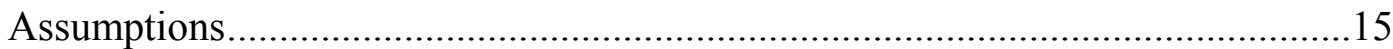

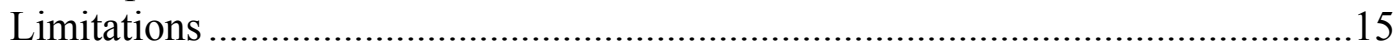

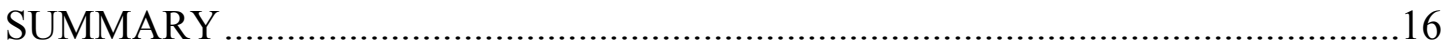

CHAPTER 2. REVIEW OF LITERATURE ................................................................18

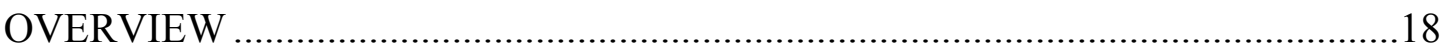

HIV IN THE U.S. POPULATION: PREVALENCE AND EPIDEMIOLOGY ..........18

HIV IN AFRICAN AMERICANS ……………………..................................19

HIV AND AFRICAN AMERICAN WOMEN …………....................................19

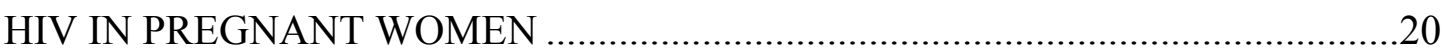

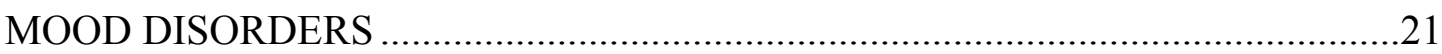

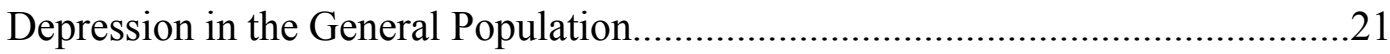

Anxiety in the General Population.......................................................................22

Co-morbid Depression and Anxiety in the General Population ..............................23

Mood Disorders in the African American Population .............................................24

Depression and Anxiety in HIV-positive Patients ...............................................25

Depression and Anxiety in African American HIV-positive Women ....................27

Depression and Anxiety in Pregnancy ...................................................................29 
INHERITED VULNERABILITIES FOR MOOD DISORDERS.................................31

Family History of Mood Disorders........................................................................

Family History of Alcohol and/or Drug Use .........................................................34

ACQUIRED VULNERABILITIES FOR MOOD DISORDERS ……….....................36

Alcohol and/or Drug Use and Mood Disorders ......................................................36

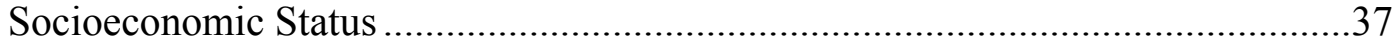

Educational Level and Mood Disorders …………...............................................38

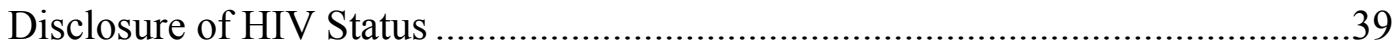

Sleep Quality and Mood Disorders....................................................................4

Sexual Assault and Mood Disorders ..............................................................42

SUMMARY OF THE REVIEW OF LITERATURE................................................43

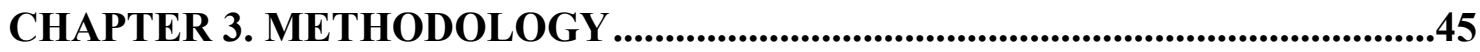

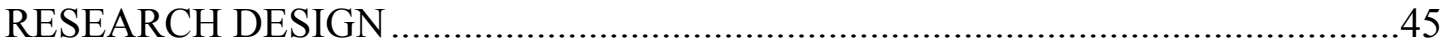

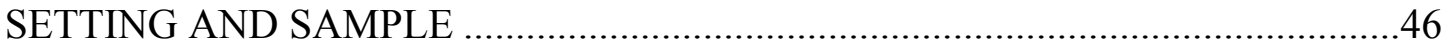

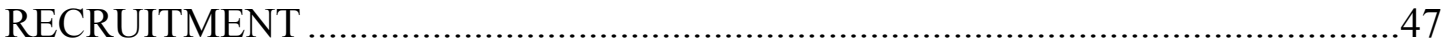

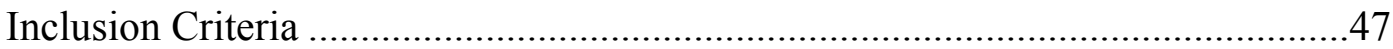

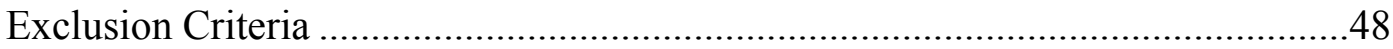

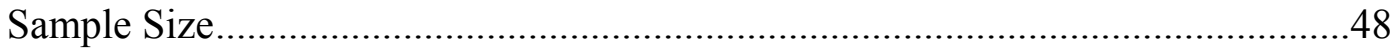

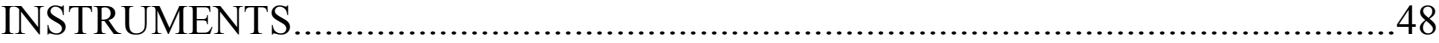

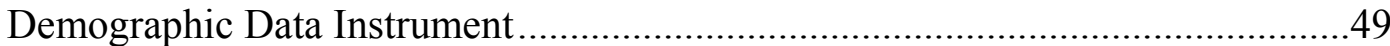

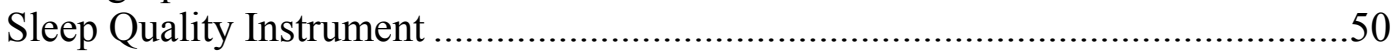

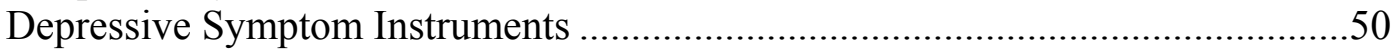

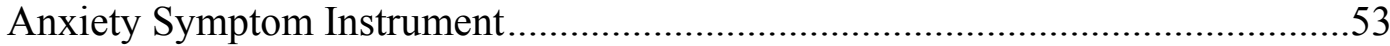

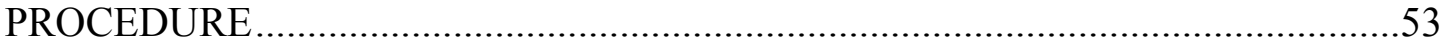

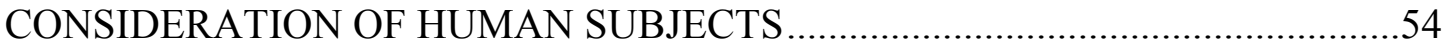

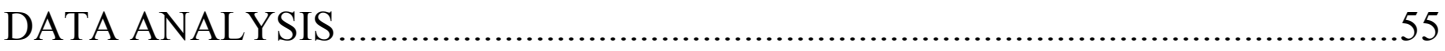

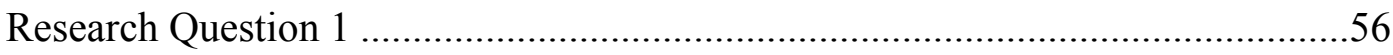

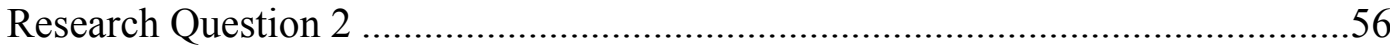

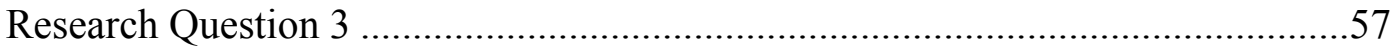

Research Question 4 ……...........................................................................57

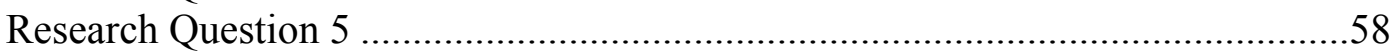

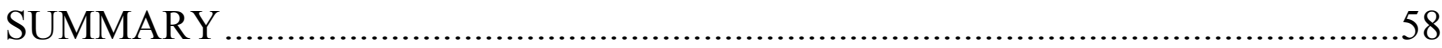

CHAPTER 4. RESULTS ..........................................................................................59

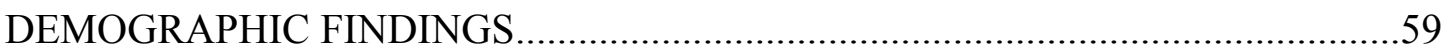

INHERITED AND ACQUIRED VULNERABILITIES...........................................60

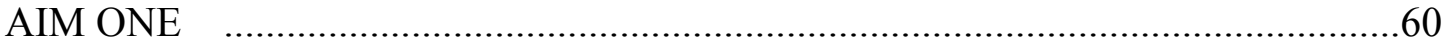

All Women: Total Scale Scores for Outcome Variables ........................................60

Pregnant Women: Total Scale Scores for Outcome Variables .................................64

All Non-Pregnant Women: Total Scale Scores for Outcome Variables...................67

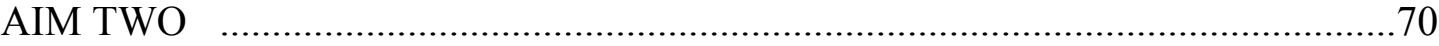

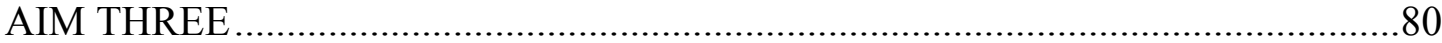

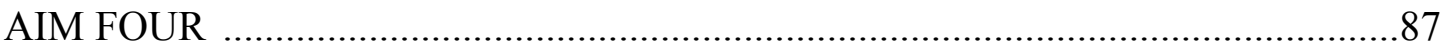


CHAPTER 5. DISCUSSION AND IMPLICATIONS .........................................103

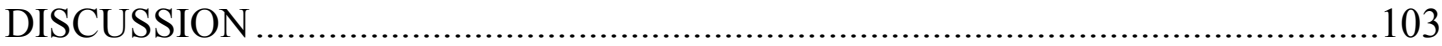

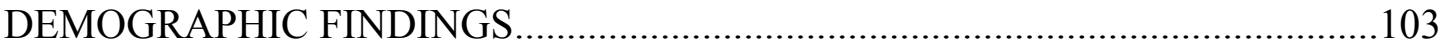

AIM ONE. LEVELS OF DEPRESSIVE AND ANXIETY SYMPTOMS ..............104

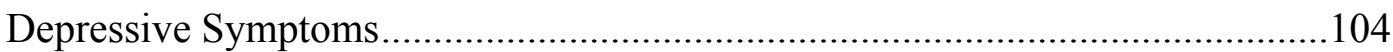

Anxiety Symptoms ..................................................................................... 105

AIM TWO. ASSOCIATIONS AMONG INHERITED AND ACQUIRED

VULNERABILITIES AND DEPRESSIVE SYMPTOMS .................106

Inherited Vulnerabilities and Depressive Symptoms........................................106

Acquired Vulnerabilities and Depressive Symptoms ........................................106

AIM THREE. ASSOCIATIONS AMONG INHERITED AND ACQUIRED

VULNERABILITIES AND ANXIETY SYMPTOMS...................108

Inherited Vulnerabilities and Anxiety Symptoms.............................................108

Acquired Vulnerabilities and Anxiety Symptoms ............................................109

AIM FOUR. PREDICTORS OF DEPRESSIVE SYMPTOMS .............................109

Inherited Vulnerabilities and Depressive Symptoms......................................109

Acquired Vulnerabilities and Depressive Symptoms .....................................110

AIM FIVE. PREDICTORS OF ANXIETY SYMPTOMS ..................................110

Inherited Vulnerabilities and Anxiety Symptoms...............................................110

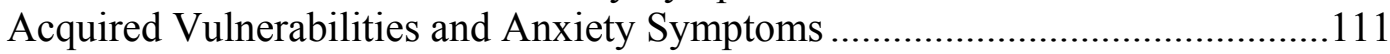

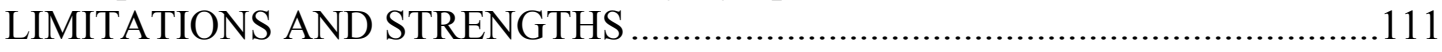

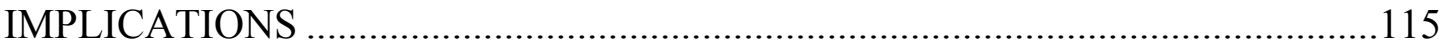

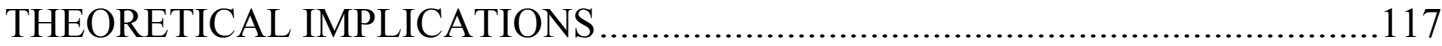

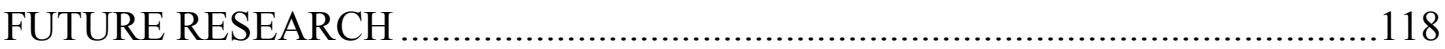

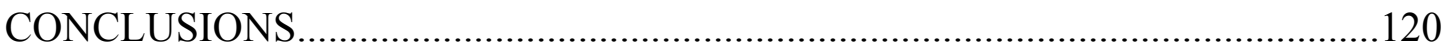

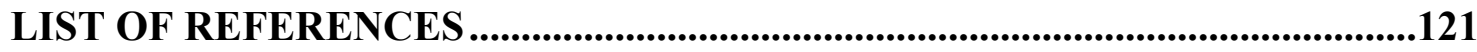

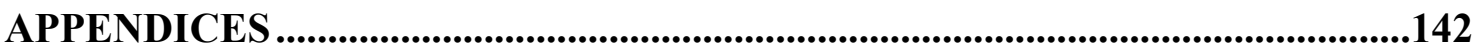

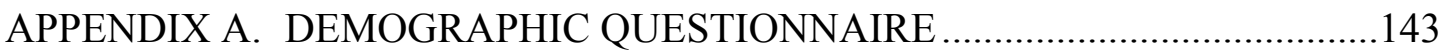

APPENDIX B. PITTSBURGH SLEEP QUALITY INDEX (PSQI) $\ldots \ldots \ldots \ldots \ldots \ldots \ldots \ldots . . . . . .147$

APPENDIX C. CENTER FOR EPIDEMIOLOGIC STUDIES DEPRESSION

SCALE (CES-D), NIMH .........................................................148

APPENDIX D. EDINBURGH POSTNATAL DEPRESSION SCALE (EPDS) ....149

APPENDIX E. INSTITUTIONAL REVIEW BOARD APPROVAL LETTER.....150

APPENDIX F. ROBERT YATES M.D. LETTER OF RECOMMENDATION ....151

APPENDIX G. MELISSA APPLETON, M. D. LETTER OF

RECOMMENDATION .......................................................152

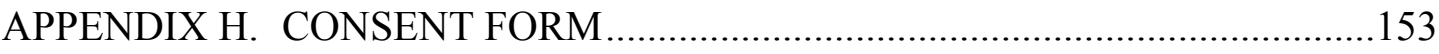

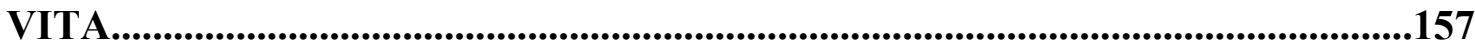




\section{LIST OF TABLES}

Table 4.1 Sociodemographic Attributes of African American Women Who Were HIV-positive.

Table 4.2 Inherited and Acquired Vulnerabilities Reported by the Total Sample and Pregnant and Non-Pregnant HIV-positive African American Women

Table 4.3 Descriptive Statistics for Depressive and Anxiety Symptoms in African American HIV-positive Women

Table 4.4 Descriptive Statistics for Specific Levels of Depressive Symptoms in Total Sample of African American HIV-positive Women ( $\mathrm{N}=80)$

Table 4.5 Descriptive Statistics for Specific Levels of Anxiety Symptoms in Total Sample of African American HIV-positive Women $(\mathrm{N}=80)$........66

Table 4.6 Descriptive Statistics for Specific Levels of Depressive Symptoms in Sample of Pregnant African American HIV-positive Women $(n=20)$

Table 4.7 Descriptive Statistics for Specific Levels of Anxiety Symptoms in Sample of Pregnant African American HIV-positive Women $(n=20)$

Table 4.8 Descriptive Statistics for Specific Levels of Depressive Symptoms in Sample of Non-Pregnant African American HIV-positive Women $(n=60)$

Table 4.9 Descriptive Statistics for Specific Levels of Anxiety Symptoms in Sample of Non-Pregnant African American HIV-positive Women $(n=60)$

Table 4.10 Depressive and Anxiety Symptom Scores in Pregnant $(n=20)$ and Non-Pregnant $(n=60)$ African American HIV-positive Women

Table 4.11 Pearson Correlation ( $r$ ) Analysis between Inherited Vulnerabilities and Depressive Symptoms for Pregnant African American Women Who Were HIV-positive $(n=20)$

Table 4.12 Pearson Correlation ( $r$ ) Analysis between Acquired Vulnerabilities and Depressive Symptoms for Pregnant African American Women Who Were HIV-positive $(n=20)$ 
Table 4.13 Pearson Correlation ( $r$ ) Analyses between Inherited Vulnerabilities and Depressive Symptoms for Non-Pregnant African American

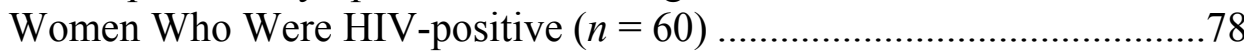

Table 4.14 Pearson Correlation ( $r$ ) Analysis between Acquired Vulnerabilities and Depressive Symptoms for Non-Pregnant African American Women Who Were HIV-positive $(n=60)$

Table 4.15 Pearson Correlation ( $r$ ) Analysis between Inherited Vulnerabilities and Anxiety Symptoms for Pregnant African American Women Who Were HIV-positive $(n=20)$

Table 4.16 Pearson Correlation (r) Analysis between Acquired Vulnerabilities and Anxiety Symptoms for Pregnant African American Women Who Were HIV-positive $(n=20)$

Table 4.17 Pearson Pearson Correlation ( $r$ ) Analysis between Inherited Vulnerabilities and Anxiety Symptoms for Non-Pregnant African American Women Who Were HIV-positive $(n=60)$

Table 4.18 Pearson Correlation (r) Analysis between Acquired Vulnerabilities and Anxiety Symptoms for Non-Pregnant African American Women Who Were HIV-positive $(n=60)$

Table 4.19 Summary of Stepwise Simple Regression Analysis by Backward Elimination for Depressive Symptom Scores Using the CES-D* among Non-Pregnant African American HIV-positive Women $(n=60)$.

Table 4.20 Summary of Stepwise Simple Regression Analysis by Backward Elimination for Depressive Symptoms Measured by EPDS* among Non-Pregnant African American HIV-positive Women $(n=60)$

Table 4.21 Summary of Stepwise Multiple Regression Analysis by Backward Elimination for Depressive Symptoms Measured by CES-D among Non-Pregnant African American HIV-positive Women $(n=60)$

Table 4.22 Summary of Stepwise Multiple Regression Analysis by Backward Elimination for Depressive Symptoms Measured by EPDS among Non-Pregnant African American HIV-positive Women $(n=60)$ .94

Table 4.23 Summary of Stepwise Multiple Regression Analysis by Backward Elimination for Anxiety Symptoms Measured by BAI among NonPregnant African American HIV-positive Women $(n=60)$ 96 
Table 4.24 Summary of Stepwise Multiple Regression Analysis by Backward Elimination for Anxiety Symptoms Measured by BAI among NonPregnant African American HIV-positive Women $(n=60)$.

Table 4.25 Collinearity Diagnostics for the Model of Inherited Vulnerabilities and Anxiety Symptoms $(n=60)$

Table 4.26 Collinearity Diagnostics for the Model of Acquired Vulnerabilities and Anxiety Symptoms $(n=60)$

Table 4.27 Collinearity Diagnostics for the Model of Depression as Measured with the Center for Epidemiologic Depression Scale and Acquired Vulnerabilities $(n=60)$

Table 4.28 Collinearity Diagnostics for the Model of Caregiver Depression as Measured with the Edinburg Postnatal Depression Scale and Acquired Vulnerabilities $(n=60)$. 102 


\section{CHAPTER 1. INTRODUCTION}

\section{OVERVIEW}

The human immunodeficiency virus (HIV), the virus that causes the fatal disease acquired immune deficiency syndrome (AIDS), is a significant health problem in the United States. The Centers for Disease Control and Prevention (CDC) reported an estimated one million HIV cases in the United States through 2003 (Branson et al., 2006; CDC, 2006). Each year, there are 40,000 new cases of HIV in the United States. HIV is the leading cause of death among Black women between the ages of 25-34 and the third leading cause of death among Black women between the ages of 35-44 (Centers for Disease Control and Prevention, 2007a).

The most common mode for HIV transmission in the United States is sexual contact, followed by parenteral exposure to contaminated blood or blood products as occurs with intravenous drug abuse or blood transfusion recipients, and lastly, between mother and fetus (Roper, 1991). In 2006, 80\% of women diagnosed with HIV in the United States were infected through heterosexual exposure (Centers for Control and Prevention, 2008; Centers for Disease Control and Prevention, 2004). Injection drug use among women accounted for $19 \%$ of cases. In addition to the direct risks associated with sharing of needles during intravenous drug use, drug use is also linked to decreased inhibitions and increased sexual risk taking, which further propagates the heterosexual spread of the epidemic (Centers for Disease Control and Prevention, 2004). Women using non-injectable drugs (i.e., cocaine, "crack", methamphetamine) are at greater risk for heterosexual HIV transmission as they trade sexual encounters for drugs or money (Centers for Disease Control and Prevention, 2004). Although the routes of transmission of the virus are known, the incidence of infection continues to increase.

The AIDS epidemic has had a major effect on morbidity and mortality in women. Studies of international AIDS research found that more women between the ages of 13 and 25 years are being infected than women in other age groups that women are infected at a much younger age than men, and that in developing countries, as many or more women are infected than men. Since 1985, the proportion of all AIDS cases reported in the United States among adult and adolescent women has more than tripled, from $7 \%$ in 1985 to 25\% in 1999 (Centers for Disease Control and Prevention, 2004). A dramatic increase in the numbers of HIV cases has been documented in women of color (Centers for Disease Control and Prevention, 2004).

The prevalence of HIV transmission among women is disproportionate, affecting racial and ethnic minorities, especially African-American and Hispanic women, at a much higher rate (Centers for Disease Control and Prevention, 2004). Of the 9708 women diagnosed with HIV in the United States in 2005, 66\% were African American (Centers for Control and Prevention, 2008). African Americans make up only 13\% of the total U. S. population, yet African Americans account for approximately $49 \%$ of the new cases of HIV (Centers for Control and Prevention, 2008). African Americans are disproportionately affected by HIV/AIDS across all stages of the disease. Sixty-three 
percent of the AIDS cases among women in the United States were among AfricanAmerican women (Centers for Disease Control and Prevention, 2004). HIV was the leading cause of death among African American women between the ages of 25 and 44 years in 2002 (Centers for Disease Control and Prevention, 2004).

Transmission patterns indicate that the rate of heterosexual transmission of HIV is increasing, directly affecting women of childbearing age. The importance of this growing epidemic among women is significant, encompassing women's physical, emotional, spiritual, and social needs. These needs are often compounded by the additional responsibility of children, some of whom may also be infected with the virus. Although the use of antiretroviral drugs in pregnancy has drastically reduced perinatal transmission of HIV, perinatal transmission accounted for 9300 cases of pediatric HIV in the United States (CDC, 2006). The burden of these needs coupled with many complex social factors increases the risk of depression and anxiety in this population. HIV-positive women with children are more likely than men to suffer from chronic sorrow related to their illness, fear of death, poverty, and social isolation (Morrison et al., 2002).

The causes of depression and anxiety have been connected to both biological and biobehavioral factors. Biological and genetic factors, including family history of mood disorders, increase the risk of depression (Gutman \& Nemeroff, 2003). Stressful life events have been linked to depression (Mazure \& Maciejewski, 2003). History of sexual abuse in depressed adolescents has been shown to increase the likelihood of psychiatric hospitalization or depressive relapse (Barbe, Bridge, Birmaher, Kolko, \& Brent, 2004). As the medical community continues to learn more about the course of HIV infection and the consequences of HIV transmission among women, the identification of depression and anxiety in this population becomes imperative. HIV has become a chronic illness, with increasing numbers of available treatment regimens, and increasing life expectancy. The denial of appropriate assessment and treatment of depressive and anxiety symptoms in this population would be neglectful and unethical.

According to the Surgeon General's Report on Mental Health (USDHHS, 1999), the current annual prevalence estimate for mental disorders is about $20 \%$ of the U. S. population. Mood disturbances are the most frequently diagnosed mental health disturbances in the U. S. and more common in persons with chronic disorders such as HIV/AIDS (USDHHS, 1999). Depression and anxiety commonly occur simultaneously. There are many studies describing either depression or anxiety, with fewer studies examining the co-morbidity of these common diagnoses.

Hirschfeld (Hirschfeld, 2001) examined the co-morbidity of major depression and anxiety in primary care settings, concluding that 10 to $20 \%$ of office visits were related to a depressive or anxiety-related episode, and over $50 \%$ of these patients experienced both depression and anxiety. Murphy and colleagues (2004) investigated the prevalence and comorbidity of anxiety and depression in the general population, reporting anxiety as twice as common as depression, and more prevalent in the female population. In a metaanalysis of studies that explored depression, Ciesla and Roberts (Ciesla \& Roberts, 2001) reported that HIV-positive persons were twice as likely to experience depression as their 
HIV-negative counterparts. African Americans may be less likely to suffer from major depression than non-Hispanic whites (USDHHS, 1999).

The prevalence of major depression in persons with HIV infection ranges from $22 \%$ to $36 \%$ (Bing et al., 2001; Ferrando et al., 1998; Rabkin, Wagner, \& Rabkin, 1999). Untreated depression along with other co-morbid conditions may increase clinic visits, hospitalizations, substance abuse, and risky behaviors, as well as reduce adherence to treatment and quality of life (Valente, 2003). Stressful life events, such as learning of the diagnosis of HIV, the death of a partner or friend with HIV, the onset of HIV-related symptoms, HIV treatment failure, and progressive disease exacerbations leading to an AIDS diagnosis may increase depressive symptoms (Valente, 2003). Like untreated depression, these stressful events may also increase alcohol and substance use (Valente, 2003) and increase levels of anxiety. Studies (Croughan, Miller, Wagelin, \& Whitman, 1982; Kosten, Anton, \& Rounsaville, 1992) have demonstrated that increased life stressors are often associated with increased injected drug and crack cocaine use, as well as increased levels of psychological distress. As stress increases, high-risk sexual behavior may escalate (Low-Beer et al., 2000).

In newly diagnosed HIV-positive patients, high rates of anxiety are experienced after learning of their diagnosis (Croughan, Miller, Wagelin, \& Whitman, 1982; Jones, Garsia, Wu, Job, \& Dunn, 1995; Krikorian, Kay, \& Liang, 1995; Rabkin, Johnson et al., 1997). Depressive symptoms among women with HIV are associated with HIV disease progression (Ickovics et al., 2001; Mayne, Vittinghoff, Chesney, Barrett, \& Coates, 1996). HIV-positive women with chronic depressive symptoms were twice as likely to die as HIV-positive women with limited or no depressive symptoms (Ickovics et al., 2001). The complex challenges of the disease and its treatments along with diseaserelated physiological changes increase the predisposition to depression and anxiety in this population (Moore et al., 1999; Valente, 2003).Depression and anxiety produce or create a more significant problem for people with a concomitant diagnosis of HIV because the feelings of loneliness, helplessness, hopelessness, and despair may lead to suicide (Valente \& Saunders, 1997).

There are many different factors that may play roles in the development of depression and anxiety in HIV-positive persons. Inherited and acquired risk factors associated with depression and anxiety, combined with side effects of antiretroviral medications, distressing events related to stigma and shame of the diagnosis of HIV and the loss of a partner through abandonment following disclosure or death all may contribute to an environment that increases the risk for depression and anxiety. Patients with HIV infection are also subject to overwhelmingly complex treatment regimens. Drug resistance and intolerance, metabolic complications and toxicities, noncompliance, and virulent strains of the disease often lead to treatment failures and regimen changes. Tsao and colleagues (Tsao, Dobalian, Moreau, \& Dobalian, 2004) reported that HIVpositive patients with HIV-related symptoms were more likely to suffer from persisting depression and anxiety or develop new cases of anxiety and depression. In addition, depression and anxiety have been related to an increase in other physical illnesses such as 
cardiac disorders, hypertension, gastrointestinal disorders, genitourinary disorders, and migraines (M. C. Harter, Conway, \& Merikangas, 2003).

\section{PURPOSE OF THE STUDY}

The incidence of HIV transmission continues to increase. The CDC reported a cumulative estimated number of 525,442 deaths of persons with AIDS through 2005 in the 50 states and the District of Columbia (Centers for Control and Prevention, 2007; Centers for Disease Control and Prevention, 2004). The prevalence of HIV infections in the heterosexual community has led to an epidemic that directly affects women of childbearing age. Women are at increased risk for major depression (Blazer, Kessler, McGonagle, \& Swartz, 1994; Blehar \& Oren, 1995; M. C. Harter, Conway, \& Merikangas, 2003; R. C. Kessler, McGonagle, Swartz, Blazer, \& Nelson, 1993) irrespective of their HIV status and at even greater risk for depression in the presence of HIV infection (Rabkin, Johnson et al., 1997). Identifying the predictors of depression and anxiety in HIV-positive women can lead to early detection and treatment of depression and anxiety in this vulnerable population. This study collected information on both pregnant and non-pregnant African American HIV-positive women. The inclusion of pregnant women is an important aspect of the study, as African American women of childbearing age represent a significant portion of new HIV cases in the United States. Pregnant women often have different needs and concerns than non-pregnant women. These differences have the potential to influence anxiety and depressive symptoms, and, thus need to be examined. Therefore, the purposes of this study of HIV-positive women are fourfold: (1) to determine the levels of depressive and anxiety symptoms in African American women who are HIV-positive; (2) to describe the underlying vulnerabilities, both inherited and acquired, for depressive and anxiety symptoms in African American women who are HIV-positive; (3) to identify which vulnerabilities may serve as predictors of depressive and anxiety symptoms among African American HIV-positive women; and (4) to describe the differences in predictors of depressive and anxiety symptoms among African American HIV-positive women.

\section{AIMS AND RESEARCH QUESTIONS}

This study was guided by the existing literature including gaps in knowledge regarding symptoms of depression and anxiety in women who are HIV-positive. Study aims were examined within one clinical population of African American women seeking treatment in two large university-based clinics that provide healthcare for women with HIV/AIDS. The specific aims and research questions that guided this study were:

Specific Aim One

Specific Aim One was to examine the levels of depressive and anxiety symptoms in African American women (pregnant and non-pregnant) who were HIV-positive in a 
southeastern urban university obstetrics and gynecology and infectious disease practice using the following questions.

a. What were the levels of depressive symptoms in pregnant African-American women who were HIV-positive?

b. What were the levels of anxiety symptoms in pregnant African-American women who were HIV-positive?

c. What were the levels of depressive symptoms in non-pregnant African-American women who were HIV-positive?

d. What were the levels of anxiety symptoms in non-pregnant African-American women who were HIV-positive?

Specific Aim Two

Specific Aim Two was to examine the associations among inherited (family history of mood disorders, family history of alcohol and/or drug use) and acquired (educational level, income level, alcohol and/or drug use, HIV disclosure status, sleep quality, history of sexual abuse or assault) vulnerabilities and depressive symptoms in African American women who were HIV-positive using the following questions.

a. What was the relationship of inherited vulnerabilities (family history of mood disorders, family history of alcohol and/or drug use) to depressive symptoms in pregnant African American women who were HIV-positive?

b. What was the relationship of acquired vulnerabilities (educational level, income level, alcohol and/or drug use, HIV disclosure status, sleep quality, history of sexual abuse or assault) to depressive symptoms in pregnant African American women who were HIV-positive?

c. What was the relationship of inherited vulnerabilities (family history of mood disorders, family history of alcohol and/or drug use) to depressive symptoms in non-pregnant African American women who were HIV-positive?

d. What was the relationship of acquired vulnerabilities (educational level, income level, alcohol and/or drug use, HIV disclosure status, sleep quality, history of sexual abuse or assault) to depressive symptoms in non-pregnant African American women who were HIV-positive?

Specific Aim Three

Specific Aim Three was to examine the associations among inherited (family history of mood disorders, family history of alcohol and/or drug use) and acquired (educational level, income level, alcohol and/or drug use, HIV disclosure status, sleep quality, history of sexual abuse or assault) vulnerabilities and anxiety symptoms in African American women who were HIV-positive using the following questions. 
a. What was the relationship of inherited vulnerabilities (family history of mood disorders, family history of alcohol and/or drug use) to anxiety symptoms in pregnant African American women who were HIV-positive?

b. What was the relationship of acquired vulnerabilities (educational level, income level, alcohol and/or drug use, HIV disclosure status, sleep quality, history of sexual abuse or assault) to anxiety symptoms in pregnant African American women who were HIV-positive?

c. What was the relationship of inherited vulnerabilities (family history of mood disorders, family history of alcohol and/or drug use) to anxiety symptoms in nonpregnant African American women who were HIV-positive?

d. What was the relationship of acquired vulnerabilities (educational level, income level, alcohol and/or drug use, HIV disclosure status, sleep quality, history of sexual abuse or assault) to anxiety symptoms in non-pregnant African American women who were HIV-positive?

Specific Aim Four

Specific Aim Four was to examine the influence of inherited vulnerabilities (family history of mood disorders, family history of alcohol and/or drug use) and acquired vulnerabilities (educational level, income level, alcohol and/or drug use, HIV disclosure status, sleep quality, history of sexual abuse or assault) on depressive symptoms in non-pregnant African American women who were HIV-positive.

a. What was the influence of inherited vulnerabilities (family history of mood disorders, family history of alcohol and/or drug use) on the level of depressive symptoms reported by non-pregnant African-American women who were HIVpositive?

b. What was the influence of acquired vulnerabilities (educational level, income level, alcohol and/or drug use, HIV disclosure status, sleep quality, history of sexual abuse or assault) on the level of depressive symptoms reported by nonpregnant African-American women who were HIV-positive?

Specific Aim Five

Specific Aim Five was to examine the influence of inherited vulnerabilities (family history of mood disorders, family history of alcohol and/or drug use) and acquired vulnerabilities (educational level, income level, alcohol and/or drug use, HIV disclosure status, sleep quality, history of sexual abuse or assault) on anxiety symptoms in non-pregnant African American women who were HIV-positive.

a. What was the influence of inherited vulnerabilities (family history of mood disorders, family history of alcohol and/or drug use) on the level of anxiety symptoms reported by non-pregnant African-American women who were HIV positive? 
b. What was the influence of acquired vulnerabilities (educational level, income level, alcohol and/or drug use, HIV disclosure status, sleep quality, history of sexual abuse or assault) on the level of anxiety symptoms reported by nonpregnant African-American women who were HIV positive?

\section{CONCEPTUAL FRAMEWORK}

A conceptual framework is defined as interrelated concepts or abstractions that are assembled together in some rational scheme by virtue of their relevance to this common theme (Polit \& Hungler, 1995). The conceptual framework that guided this study served to organize the empirical data, formulate the research questions, select the study instruments and interpret study findings. Furthermore, the conceptual framework aided in the exploration of existing knowledge gaps, which may facilitate development of future theories that might improve understanding of the factors that predict depressive and anxiety symptoms in the study population, and strategies to improve patient outcomes. The proposed study investigated inherited and acquired vulnerabilities as predictors of depressive and anxiety symptoms among African American HIV-positive women.

The stress diathesis model proposes that vulnerability factors contribute to the onset of depression when there is an interaction between these factors and negative life events (A. T. Beck, 1967; Monroe \& Simons, 1991). According to this model, individuals form a psychological vulnerability to depression through stressful life events that influence the development of negative cognitive schemas (Sacco \& Beck, 1995). Predispositions to mood disorders are developed as negative schemas become latent during cycles of recovery but have the potential to be reactivated. The cascade of events begins when a traumatic event, or event that is reminiscent of a previous trauma, occurs and thereby reactivates the negative cognitive process, leading to negative experiences and symptoms (Sacco \& Beck, 1995).

Some persons, even under conditions of extreme stress, do not experience a diathesis that leads to mental health compromise (B. P. Dohrenwend \& Dohrenwend, 1979). All individuals experience daily and chronic stress, all with different backgrounds

and predispositions, resulting in different responses to stressors. The concept of allostatic load plays an integral part in the stress diathesis model. Sterling and Eyer (Sterling \& Eyer, 1998) identified allostasis as the ability to achieve stability through change. There is a broad range of behavioral responses to stressful life events. Positive outcomes, such as coping, are the result of successful adaptation to stressors. It is when the body is unable to adapt to stressful life events that injury occurs. McEwen (Bruce S. McEwen, 2002) explored the relationship of allostasis and allostatic load on mood disorders. Allostasis (Bruce S. McEwen, 2002) refers to the adaptation that occurs as a response to stress. The process is complicated when adaptation is altered, either for biological or situational reasons, resulting in an allostatic state. An allostatic state results when there is the failure to turn off mediators after stress (Bruce S. McEwen, 2002). When adaptation fails, the result is injury. Cumulative effects of stress may be the result of repeated failures in adaptation. Allostatic load is the long-term effect of repeated injury to the 
body. McEwen (Bruce S. McEwen, 2002) describes four situations associated with high allostatic load: (1) frequent stress, (2) repeated stressors, (3) inability to turn off allostatic responses after the stress is over, and (4) the trigger of compensatory responses due to inadequate allostatic responses. Increased allostatic load has an effect on virtually every body system, resulting in impaired immunity, obesity, atherosclerosis, and bone demineralization (Bruce S. McEwen, 2002). Prolonged allostatic load increased the risk for permanent damage to the brain in rats (Lowy, Wittenberg, \& Yamamoto, 1995). Allostatic load can be caused by genetic or developmental disturbances that prevent adequate coping. Individual responses to daily stressors are influenced by neurobiological factors, which effect the interpretation and response to stress (Bruce S. McEwen, 2002).

Hans Selye was one of the first scientists to explore the physiologic stress response. Selye (1976) noted that the secretion of excess adrenocorticotrophic hormone by the pituitary gland results in an increased release of glucocorticoids from the adrenal cortex. This classic neuroendocrine response is present during both positive and negative periods of stress (Selye, 1974). Excessive hypothalamic-pituitary-adrenal and nonadrenergic system activity and dysfunction of the serotonergic system play a role in the pathophysiology of suicidal behavior (van Heeringen, 2003). The effect of stressful life events on neurobiological systems and the dysfunction of neurobiological systems have been linked to suicidal behavior, impulsivity, aggression, and increased levels of anxiety (van Heeringen, 2003).

Stressful life events, whether acute or chronic, lead to increased cortisol secretion in both healthy and depressed persons (B. S. McEwen, 2006; Sher, 2004). The amount or level of stress cannot predict outcomes, as there are individual variations in responses, and the theory that some people have underlying vulnerabilities to depression and other mood disorders. The prevention of stress is not feasible; therefore successful coping strategies are suggested in order to maintain homeostasis in stressful situations.

The physiological stress response has been correlated with lifestyle. Early adverse experiences (Gutman \& Nemeroff, 2003) and sleep deprivation (McEwen, 2006; McEwen \& Wingfield, 2003) affect the physiologic stress response. Early adverse experiences may sensitize corticotropin-releasing factor processes (Gutman \& Nemeroff, 2003). Depression in adults may also be linked to the disruption of neurological pathways caused by early adverse life experiences. Poor quality of sleep or disruptions of sleep patterns may result in hypothalamic-pituitary-adrenal axis dysregulation, while short periods of sleep deprivation have been shown to cause increased levels of cortisol, glucose, insulin, and insulin resistance (B. S. McEwen, 2006; Plat et al., 1999). Hypercortisolism has been linked depressive disorders (Sher, 2004).

Increased allostatic load can result in adverse outcomes. The physiologic changes that occur as a result of increased allostatic load may affect the immune system, resulting in the expression of inflammatory, infectious, and autoimmune diseases (United States Department of Health and Human Services [USDHHS], (USDHHS, 1999). Long term effects of chronic depression may be associated with increased allostatic load, resulting in pathophysiologic changes (B. S. McEwen, 1998). There is a potential for adverse cardiac 
outcomes and increased platelet reactivity with concomitant long-term increased allostatic load and major depression (Heuser, 2002; Lederbogen et al., 2001; Thayer, Smith, Rossy, Sollers, \& Friedman, 1998).

Although the precise etiology of mood disorders is unknown, there is evidence that the interplay of biological, psychological, social, and cultural factors results in mood disorders for some individuals (USDHHS, 1999). There is increased evidence that there are biologic and physiologic causes of mood disorders. Responses to stressful life events may vary. However, the body's successful adaptation to stress results in allostasis, appropriate responses, and successful coping. It is when the body is unable to adapt to stressful life events that mood disorders are often expressed (Bruce S. McEwen, 2002). Prolongations of stressors that result in a physiologic stress response have the potential to cause adverse outcomes.

Although it is impossible to predict who will develop a mood disorder, the assessment of lifestyle, individual and family history, and presence and type of current stressors is an important aspect of evaluation of mood disorders. Thus, in the current study, these underlying vulnerabilities were examined as potential predictors of depressive and anxiety symptoms. Underlying inherited vulnerabilities that were examined in this study included family history of mood disorders and family history of alcohol and/or drug use. Underlying acquired vulnerabilities assessed in the current study included educational level, income level (annual household income), alcohol and/or drug use, HIV disclosure status, sleep quality, and history of sexual abuse or assault.

The evaluation of potential predictors of psychopathology is essential in the comprehensive care of women with HIV infection. Healthcare providers often overlook the importance and prevalence of depression and anxiety in this population. However, all persons have underlying vulnerabilities, both inherited and acquired, which have the potential to trigger a cascade of events resulting in disrupted mental processes.

The stress diathesis model appropriately describes and explains this process, therefore serving as an appropriate conceptual framework for this study. The stress diathesis model has been used to understand depressive and anxiety in this population (Ickovics et al., 2001), and has been shown to be useful for understanding depression and anxiety in general (Monroe \& Simons, 1991). Therefore, this study tested the utility of the stress diathesis model in this population. Figure 1.1 depicts the stress diathesis model as it applied to the conceptual framework of the current study.

\section{Definition of Major Concepts}

A variety of theoretical and operational definitions have been published for the concepts of interest within the current study. For the purpose of the current study, the following definitions were used in order to provide conceptual consistency and clarity. 


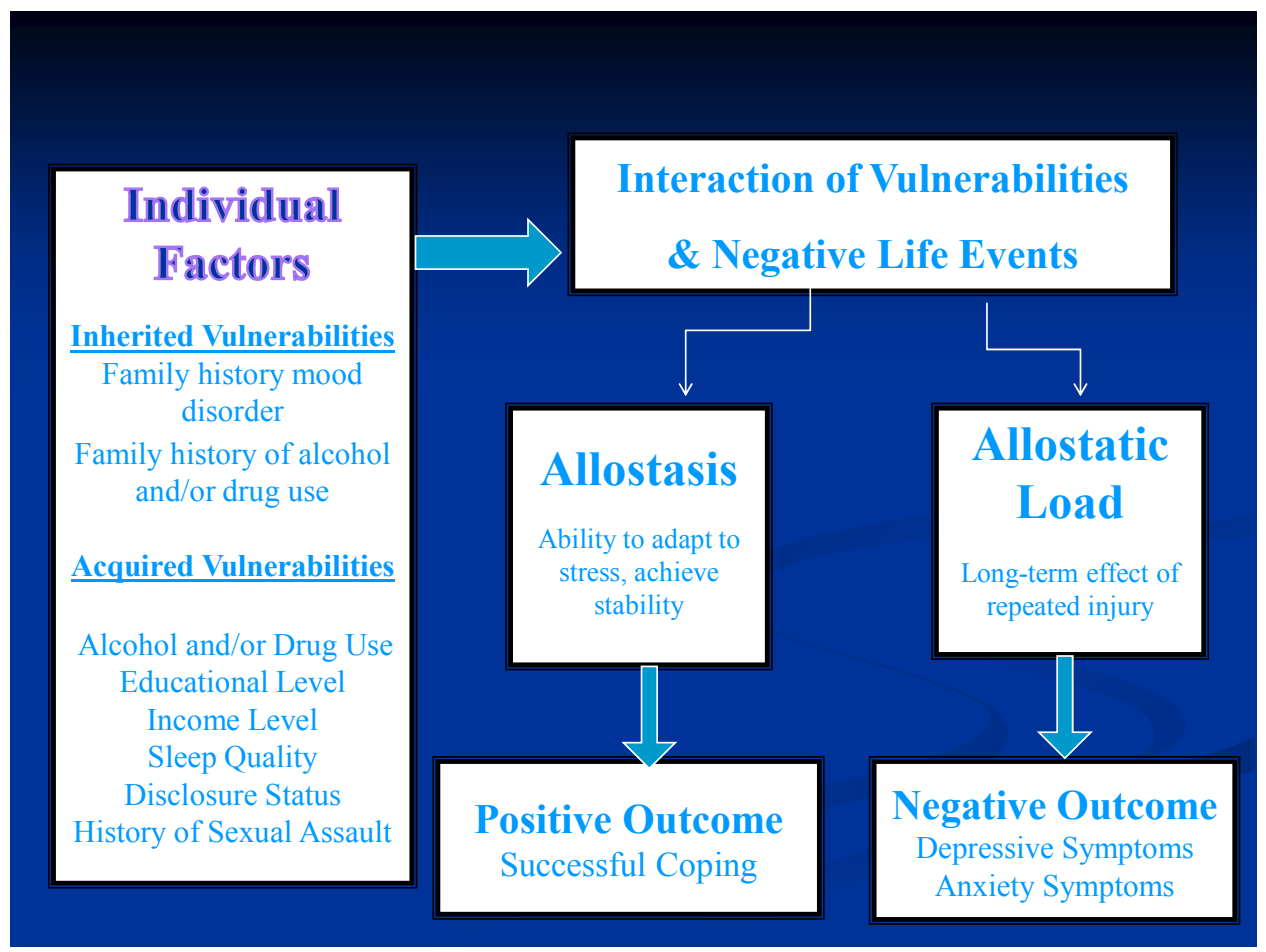

Figure 1.1 Conceptual Framework Depicting the Stress Diathesis Model with Inherited and Acquired Vulnerabilities. 


\section{$\underline{\text { HIV-positive women }}$}

In this study, the theoretical definition of $H I V$-positive women is women that have been diagnosed with the human immunodeficiency virus by their health care professional with positive HIV antibody testing, including the HIV-1 ELISA and HIV-1 Western Blot. The diagnosis of HIV was confirmed by medical chart abstraction. This concept was quantified as English-speaking African American females who were over the age of 18 years, who had been diagnosed with HIV, and were receiving care at a university outpatient obstetrics and gynecology clinic or outpatient infectious disease clinic.

\section{African American}

The theoretical definition for African American is pertaining to or characteristic of Americans of African ancestry (Mish, 1997). The definition of African American will be operationalized through self-report. Participants must have lifelong residence in the United States in order to eliminate the influence that cultural differences might play in the interpretation of study instruments.

\section{Depression}

Depression refers to a reduction of the level of functioning; a sinking of spirits so as to constitute a clinically discernible condition (Hensyl, 1990). The NIMH (National Institute of Mental Health, n/d) describes three main types of depression: dysthymia, major depression, and bipolar disorder. The operational definition of depression that was used for this study was Center for Epidemiologic Studies Depression Scale (Radloff, 1977) or Edinburgh Postnatal Depression Scale (Cox, Holden, \& Sagovsky, 1987) scores at or above the recommended cut-off for additional evaluation. No attempt was made to assign a diagnosis to participants' symptoms. The study described the level of depressive symptoms that were present in study participants and compared their symptoms with other populations.

\section{Anxiety}

The theoretical definition of anxiety is an apprehension of danger and dread accompanied by restlessness, tension, tachycardia, and dyspnea unattached to a clearly identifiable stimulus (Hensyl, 1990). The NIMH (National Institute of Mental Health, $\mathrm{n} / \mathrm{d}$ ) defines generalized anxiety disorder as a chronic disorder, which results in feelings of impending disaster, often related to money, health, family, or work. Sources of anxiety are not always identifiable. The operational definition of anxiety that was used for this study was a positive score on the Beck Anxiety Inventory (A. T. Beck, Epstein, Brown, $\&$ Steer, 1988). The proposed study instrument did not serve as a tool for diagnosing anxiety, but rather as a means to assess the level of anxiety symptoms reported by study participants and therefore determine the need for further assessment of anxiety symptoms in this population. The proposed study findings will provide additional insights that may guide recommendations for future assessments in this population. 
General Definitions

$\underline{\text { Health }}$

Health is defined as a state of complete physical, mental and social well being and not merely the absence of disease or infirmity (World Health Organization, 1992). Health can be defined as a state (at one time) or a continuum (over a longer period of time). This study used a comprehensive definition of health that included both mental and physical health, and was measured through self-reporting of subjective symptoms, and documentation of disease markers (CD4 count), which were obtained through medical record chart abstraction.

\section{$\underline{\text { Mental Health }}$}

Mental health is defined as emotional, behavioral, and social maturity or normality; the absence of a mental or behavioral disorder; a state of psychological wellbeing in which the individual has achieved a satisfactory integration of his instinctual drives acceptable to both himself and his social milieu (Hensyl, 1990). For the purposes of the proposed study, mental health was theoretically defined as the scores on the CESD (Radloff, 1977), EPDS (Cox et al., 1987), and Beck Anxiety Inventory (A. T. Beck et al., 1988).

\section{$\underline{\text { Inherited Vulnerabilities }}$}

The term inherit can be defined as to receive from one's ancestors (Mish, 1997). Vulnerability can be defined as susceptibility to wounds, or capability of being wounded (Mish, 1997). In the current study, the term vulnerabilities referred to susceptibility to mood disorders. In the current study, inherited vulnerabilities were defined as family history of mood disorders and family history of alcohol and/or drug use. The underlying predispositions to biologic and familial manifestations of mood disorders are key concepts in the stress diathesis model. This definition is consistent with components of the stress diathesis theory that serves as the conceptual framework for this study. The inherited vulnerabilities of the sample were individual characteristics that were determined through self-report.

\section{$\underline{\text { Acquired Vulnerabilities }}$}

The term acquired can be defined as gained by or as a result of effort or experience (Mish, 1997). Vulnerability can be defined as susceptibility to wounds, or capability of being wounded (Mish, 1997). In the current study, acquired vulnerabilities were defined as educational level, income level, alcohol and/or drug use, HIV disclosure status, sleep quality, and history of sexual abuse or assault. This definition is consistent with the stress diathesis theory that serves as the conceptual framework for this study. These acquired vulnerabilities of the sample were individual characteristics that were determined through self-report. 
Concept Relationships

The identification of inherited and acquired vulnerability cannot solely predict depressive or anxiety symptoms in a person. There are many different variables that influence mental health, all of which may never be identified. Variables included in inherited vulnerability are the following: family history of mood disorders, and family history of alcohol and/or drug use. Researchers (Moore et al., 1999; Perkins et al., 1994; Rabkin, Ferrando, Jacobsberg, \& Fishman, 1997) have documented an increased prevalence of depression among HIV-positive women (30-60\%), when compared to HIVpositive men ( $20 \%$ or less). Researchers have demonstrated that minority status is often associated with poverty and lower socioeconomic status (Wilhelm \& Parker, 1994), and that lower socioeconomic status is associated with greater risk for mood disorders (USDHHS, 1999). Minority women are at greater risk for feelings of isolation and alienation, which may lead to unhealthy behaviors and coping mechanisms, therefore increasing their risk for depressive symptoms (Boyd-Franklin, 1987). Family history of mood disorders has also been correlated with adult depression and anxiety (Gutman \& Nemeroff, 2003).

Variables included in acquired vulnerability are the following: educational level, income level, alcohol and/or drug use, HIV disclosure status, sleep quality, and history of sexual abuse or assault. Moore and colleagues (Moore et al., 1999) reported that disadvantaged socioeconomic and environmental backgrounds were more related to depressive symptoms than HIV infection. Adverse life events are also predictors of depressive symptoms in women with HIV (Moore et al., 1999). Insomnia has been associated with psychiatric disorders, including depression (Lustberg \& Reynolds, 2000). The assessment of sleep patterns, sleep preparation practices (also known as sleep hygiene), sleep quality and duration of sleep are important aspects of comprehensive sleep assessments. The relationship of sleep disturbances to depression and anxiety has been documented. Socioeconomically disadvantaged women, regardless of HIV status, have many barriers and adversities that make health maintenance and promotion difficult (Moore et al., 1999). The identification of reported histories of sexual abuse or assault is pertinent to the assessment of mental health. The relationship between a history of child sexual abuse and adult depression and anxiety has been well documented (Barbe et al., 2004; Barker-Collo, Read, Barker-Collo, \& Read, 2003; Walrath et al., 2003). Adult sexual abuse or assault has been associated with increased feelings of sadness and dysphoria (M. Conway, Mendelson, Giannopoulos, Csank, \& Holm, 2004).

Depression has been explained through several cognitive stress diathesis models in the literature (Hilsman \& Garber, 1995; Monroe \& Simons, 1991). The intersection of underlying predispositions with a major life stressor has the potential to activate a cascade of events that result in psychopathology (Monroe \& Simons, 1991). The identification of persons with vulnerabilities to mental illness is essential in evaluating risks of a major mental health disorder. Although the presence of predispositions to psychopathology will not always activate the diathesis that results in a significant event, it is important to identify the underlying predispositions in persons at greater risk for depression and anxiety. Gender and the presence of a HIV diagnosis have been documented (Ciesla \& Roberts, 2001; J. M. Murphy et al., 2004; National Institute of 
Mental Health, n/d) to have a direct impact on increased levels of depressive symptoms. Therefore, the evaluation of underlying predispositions, or vulnerabilities, is critical to monitoring the potential onset of depressive symptoms, as well as symptoms of anxiety in the study population.

\section{SIGNIFICANCE OF STUDY}

Although the routes of HIV transmission are well documented and prevention inexpensive (i.e. abstinence, condom use), the rates of HIV cases and heterosexual transmission of HIV continue to increase. Women of childbearing age are at a greater risk for depression and anxiety than the general population. The cost of mental illness in the United States is staggering, ranking second to cardiovascular conditions in the burden of disease in established market economies (USDHHS, 1999). Depression can lead to poor self-esteem, feelings of hopelessness, and suicide. "Mental problems affect and are affected by chronic conditions such as cancer, heart disease, diabetes and HIV/AIDS. Untreated, they bring about poor health behaviour, non-compliance with prescribed medical regimens, diminished immune functioning, and negative disease outcomes" (World Health Organization, 2004).

The identification of depressive and anxiety symptoms among HIV-positive women is imperative and relevant to nursing practice for the following reasons: (1) to identify the levels of depressive and anxiety in one clinical population; (2) to provide the preliminary evidence as to whether there is a need for implementing early screening and subsequent evaluation, diagnosis, and treatment of depressive and anxiety symptoms in this vulnerable population; and (3) to provide an environment for the developing and implementing of therapeutic interventions. The findings of this study may give practitioners cause to be aware of potential inherited and acquired vulnerabilities that increase the risk for mood disorders such as depression and anxiety among their female African American patients who are HIV-positive. It is through the identification of predictors of depressive and anxiety symptoms, that the proper screening, diagnosis, and treatment of depression and anxiety can be instigated. The findings from this study identified the relationship between the research variables and depressive and anxiety symptoms in one sample, which addressed gaps in existing literature related to this area of inquiry.

Nurse clinicians have the unique opportunity to influence their environment through research, education, and practice. The knowledge gained from this research study added to the existing information regarding HIV-infected women. This research study is significant, in that there are few studies describing the predictors of depression and anxiety among HIV positive women in general and African American HIV-positive women in particular. This research study may provide the baseline information, which can lead to early identification and treatment of anxiety and depression in HIV-positive women.

Nurse clinicians must provide education to not only their patients, but also to their co-workers and communities regarding HIV transmission, depression, and anxiety. 
Although HIV, depression, and anxiety are subjects that are avoided in many social situations, nurses have the opportunity to bridge the gap between science and practice. The identification of depressive and anxiety symptoms in HIV-positive women is critical to long-term success of antiretroviral treatment and adherence to medical appointments. The long-term ramifications of the identification, assessment, and treatment of depressive and anxiety symptoms in HIV-positive women include decreased health care costs related to decreased numbers of inpatient hospitalizations and increased longevity of successful highly active antiretroviral treatment (HAART) regimens. Successful long-term HAART treatment leads to better patient outcomes as patients experience complete virologic control and immune reconstitution, as well as decreased transmission of drug resistant viruses (Havlir \& Richman, 1996). Depression has been associated with poor adherence to medication regimens among HIV-positive persons (Gordillo, del Amo, Soriano, \& Gonzalez-Lahoz, 1999; Starace et al., 2002; Wagner et al., 2006).

It is imperative that nurses explore the predictors of depression and anxiety among their HIV-positive clients. This information is vital to the development of counseling strategies and treatment interventions that can be implemented in order to maintain stable mental and physical health in these patients with complicated health care needs.

Assumptions

The following assumptions for this study were used in the development of study framework, design, and interpretation of the results. The assumptions surrounding this study are the following:

1. Women who agree to participate in the study are aware of their family history of depression and anxiety. Several researchers have described the influence of genetic factors and family history on depression (Gutman \& Nemeroff, 2003; Reinherz et al., 2003).

2. Women who agree to participate in the research study will be able to read and understand the study's instrumentation.

3. Women who participate in the study will answer the instruments honestly.

4. Positive HIV status is a stressor for the subjects in this sample.

5. The concepts in the study framework are relevant for the study sample and population.

\section{Limitations}

The investigator for this research study identified several potential limitations. These limitations were methodological in nature. The techniques used to minimize them are discussed.

1. Non-probability sampling (convenience sampling) was used and consisted of HIV-positive women receiving care at university clinics. This sampling method 
contributed to a weaker design than if random sampling had been utilized. A random sampling procedure increases the likelihood of generalizability by guaranteeing that each unit of the population has a non-zero chance of selection (Hulley, 2001). A convenience sampling method was appropriate due to the limited existing body of knowledge regarding the subject matter and economic considerations that alternative sampling methods required. The use of nonprobability sampling limits external validity by limiting the generalizability of the findings to target populations (Penhazur \& Schmelkin, 1991). The sample was entirely composed of HIV-positive women engaged in medical care, thus decreasing the generalizability to African American female HIV-positive populations who are not receiving medical care.

2. The potential inability of the subjects to honestly report symptoms of depression and anxiety was another limitation of the study. The stigma that is often associated with mental health issues may encourage subjects to underreport symptoms. The questionnaires did not contain subject identifiers, which should have encouraged truthful reporting. LoBiondo-Wood and Haber (Lo-BiondoWood \& Haber, 1994) suggest that anonymity may be important when the study deals with sensitive issues.

3. Women who are severely depressed may not be represented in the study as their levels of depression may have prevented them from accessing care or participating in the study.

4. Subjects were at different stages of their disease, and had different durations of diagnosis. Data were collected during only a single time point, therefore decreasing the external validity of the findings. The identification of different levels of severity of depressive symptoms during different stages of disease and length of diagnosis will, however, add to the body of existing literature regarding HIV-positive women.

5. The women accessed their care in a metropolitan area, thus the results may not be generalized to similar populations living in rural settings.

6. Socioeconomic and demographic characteristics of the sample may differ from the HIV-positive women that seek care in private practice facilities.

\section{SUMMARY}

The prevalence of HIV infection among women has become a significant health problem in the United States. The numbers of heterosexually transmitted infections have increased significantly in the last decade (Centers for Control and Prevention, 2007). Despite education regarding HIV prevention, the numbers of new infections continue to rise. Women of childbearing age represent a substantial number of new HIV infections each year, and the rates continue to climb among minority women (Centers for Control and Prevention, 2007; Centers for Disease Control and Prevention, 2005, 2007b). 
Women who have been diagnosed with HIV have compounding issues that often complicate the course of the disease. Women often experience additional stressors such as the following: stigma, isolation, and shame that is often associated with HIV; loss of family or partner support following disclosure of HIV status; responsibilities of being the primary caregiver to children; guilt associated with potential mother to child HIV transmission; and anxiety regarding permanency planning for their children. When these stressors are combined with the diagnosis of HIV, there may be an increase in experiencing significant depression and anxiety. Women are often burdened with additional stressors such as single parenthood, caring for children and/or aging parents, work and home responsibilities (Barroso, Pence, Salahuddin, Harmon, \& Leserman, 2008).

Women experience hormonal factors that occur during menstrual cycle changes, pregnancy, miscarriage, postpartum period, pre-menopause, and menopause, all of which may contribute to the increased rate of depression in women (Barroso et al., 2008). Physiological and or genetic factors have also been associated with depressive disorders, and may have impacted the development of depression and anxiety (Barroso et al., 2008). Therefore, it is important that both pregnant and non-pregnant women be included in studies. The inclusion of pregnant and non-pregnant women will add to the body of knowledge regarding women with HIV.

In this chapter, the problem, purpose of the study, aims and research questions, definition of terms, conceptual framework, concept relationships assumptions, limitations, significance of study, and significance to nursing were discussed. The next chapter presents the review of literature. 


\section{CHAPTER 2. REVIEW OF LITERATURE}

\section{OVERVIEW}

Much of the earlier published literature regarding depression and HIV has focused on HIV-positive men (Atkinson et al., 1988; Perkins et al., 1994; Rabkin, Goetz et al., 1997), homosexual men (Atkinson et al., 1988; Rabkin, Goetz et al., 1997), and populations of substance abusers (Lipsitz et al., 1994; Rabkin, Johnson et al., 1997), reflecting the original transmission patterns of the virus observed in the United States. Current epidemiologic trends illustrate that African American women of childbearing age are the fastest growing group of newly diagnosed HIV infected persons in the United States (Centers for Disease Control and Prevention, 2005 2007c). More recently published literature about persons who are HIV positive focuses on the impact of depression on morbidity and mortality in this population. A substantial proportion of this literature documents the negative consequences of depressive symptoms on adherence to HAART and medical appointments. Few studies examine the multiple inherited and acquired vulnerabilities thought to influence the development of both depressive and anxiety symptoms in this population. Although the current study focuses on African American women who are HIV-positive, women in general may share similar backgrounds and experiences that contribute to their vulnerability for mood disorders. Therefore, within this chapter, specific attention will be given to describing inherited and acquired vulnerabilities that increase the risk for depression and anxiety symptoms in a sample of African American women who are HIV-positive. However before exploring these vulnerabilities, the prevalence and epidemiology of HIV in the United States within the general population and in specific populations will first be discussed followed by a review of mood disorders in the general population and in specific populations.

\section{HIV IN THE U.S. POPULATION: PREVALENCE AND EPIDEMIOLOGY}

Research indicates that despite prevention education, prevalence rates for HIV infection continue to increase in the United States. Surveillance data, although comprehensive, can only provide an estimate of overall cases. The CDC began collecting statistics on AIDS cases at the beginning of the epidemic, but currently collects surveillance data for HIV. This process provides a more comprehensive illustration of the epidemic, as earlier data did not account for healthy persons with HIV, who had not yet developed AIDS. Reporting requirements vary by state; therefore, CDC surveillance data only includes information from 33 states with confidential name-based HIV infection reporting (Branson et al., 2006).

Approximately one million people are living with HIV in the United States (Branson et al., 2006), including 250,000 persons that have not yet been diagnosed. Surveillance data for HIV in the United States includes descriptions of cases by age, race/ethnicity, sex, and transmission category. The prevalence and epidemiology of HIV on African Americans and African American women, as well as HIV within the context of pregnancy will be discussed in the next sections. 


\section{HIV IN AFRICAN AMERICANS}

More than half of new HIV infections (51\%) in the United States occur in African Americans (Centers for Disease Control and Prevention, 2007c). In general, minority populations in the United States are disproportionately affected by HIV. African Americans make up $12 \%$ of the total U.S. population, yet account for over $50 \%$ of the new infections each year (Centers for Disease Control and Prevention, 2007c). The rapid spread of HIV infection in African American communities has been accentuated by many factors including poverty, racism, lack of education, barriers to health care, illicit drug use, and high rates of sexually transmitted infections (Coleman, 2003; Mayberry, Mili, \& Ofili, 2000; Plowden \& Young, 2003; Treadwell \& Ro, 2003).

The stigma that is associated with HIV combined with the mistrust of governmental research and educational programs in African American communities have catapulted HIV to one of the leading causes of death among African Americans (CDC, 2006; Centers for Disease Control and Prevention, 2007b; Coleman, 2003; Plowden, \& Young, 2003; Treadwell, \& Ro, 2003). The myth that HIV is a homosexual disease has hindered HIV prevention efforts in African American communities and created a culture of silence (Beatty, Wheeler, \& Gaiter, 2004). This cycle has been counterproductive, resulting in the perpetuation of myths and increases in compensatory sexual behavior (marrying or having sex with the opposite sex) to prove one's heterosexuality (Joint United Nations Programme on HIV/AIDS [UNAIDS], (UNAIDS, 2000). This sequence of events has resulted in discrimination and rejection of HIV-positive persons in African American communities. Moreover, HIV-related shame and stigma has created another minority within a minority population.

\section{HIV AND AFRICAN AMERICAN WOMEN}

HIV is now the leading cause of death among African American women 25 to 34 years of age in the United States (CDC, 2006; Centers for Disease Control and Prevention, 2007a); however risk factors for HIV transmission vary by region. Among women and adolescent females with AIDS, the most prevalent risk factor in the Northeast was intravenous drug use, while heterosexual contact was the most prevalent risk factor in the South (Centers for Control and Prevention, 2007). Although the primary focus of the current study centered on African American women, minorities are grossly underrepresented in much of the research literature. Minorities, both men and women, are also under represented in HIV- related research studies nationwide.

Significant psychosocial issues facing African American HIV-positive women are cited in the literature and include: poverty, low levels of education, stressors related to caretaking roles, barriers to healthcare, depression, and poor access to quality healthcare (Bunting, Bevier, \& Baker, 1999; Centers for Disease \& Prevention, 2007; Centers for Disease Control and Prevention, 2005, 2007b; Heslin et al., 2005; Hu, Byers, Fleming, \& Ward, 1995; Washington, 2001). Women respond to the needs of their families, which may result in a lack of self-care, often leading to negative health consequences.

Researchers reported that African American women who were HIV-positive were more 
likely to have a family member with HIV than White American women who were HIVpositive (Fiore et al., 2001). The result of family caretaking burden experienced by African American HIV-positive women may impact their level of HIV care. Literature reveals that caretaker burden affects the overall quality of life of HIV-positive women, as the care of a HIV-infected child or partner often takes precedence over the care of the woman who is HIV-positive (Fiore et al., 2001). As the rates of HIV continue to increase among women of childbearing age, the impact of HIV on pregnancy becomes critical. HIV in pregnant women will be discussed in the next section.

\section{HIV IN PREGNANT WOMEN}

Transmission patterns in the United States indicate increased heterosexual transmission of HIV, with rising rates of infection documented in women. The prevalence of HIV among women in the United States has directly affected women of childbearing potential, with the majority of new infections documented in women between the ages of 25 to 44. The proportion of HIV/AIDS cases among women and adolescent girls (age 13 and older) increased by 14\% between the years 1986 to 2001 (Centers for Disease Control and Prevention, 2003). The concentration of infections among females aged 15 to 44 years directly impacts women during their highest years of fertility, thereby having an impact on pregnancy.

Transmission of HIV from mother to child has greatly decreased since the advent of antiretroviral therapy, with initial decreases noted with Zidovudine monotherapy (22.6\% to $7.6 \%$ ) and even further reductions with HAART (Centers for Control and Prevention, 2002). As women live longer, healthier lives with successful HIV treatment, an increasing proportion of HIV-positive women are becoming pregnant (Cohan, 2003). Although pregnancy has been associated with decreases in absolute CD4 cells, no studies have indicated that pregnancy causes an increased rate of disease progression, HIV viral load, or death (Centers for Disease Control and Prevention, 2002). Despite these hopeful findings, HIV-positive women often view themselves as "bad mothers" or "bad women for wanting to become mothers" (Sandelowski \& Barroso, 2003).

Much of the literature regarding HIV and pregnancy is related to HIV treatment. Antiretroviral distribution and metabolism is different in women when compared to men, as women have higher percentages of body fat and lower percentages of lean muscle mass than men (Anderson, 2002; Hader, Smith, Moore, \& Holmberg, 2001). Limited pharmacokinetic studies have addressed sex-related differences in regard to metabolism of some antiretroviral agents in non-pregnant women (Anderson, 2002). Physiologic changes that occur during pregnancy may change the pharmacokinetic profiles of some antiretroviral agents (Anderson, 2002; Mirochnick, 2000), thereby prompting the need for additional pharmacokinetic studies in pregnant women with HIV. No studies were found in the literature describing the impact of pregnancy on depressive and anxiety symptoms in HIV-positive women. Mood disorders, including depression and anxiety will be discussed in the next sections. 


\section{MOOD DISORDERS}

According to the Surgeon General's Report on Mental Health (USDHHS, 1999), the current annual prevalence estimate for mental disorders is about $20 \%$ of the U. S. population. Mood disturbances are the most frequently diagnosed mental health disturbances in the U. S. and more common in persons with chronic disorders such as HIV/AIDS (Barroso et al., 2008; National Institute of Mental Health, n/d). In primary care settings, an estimated 10 to $20 \%$ of office visits are related to depressive symptoms or an anxiety-related episode (Hirschfeld, 2001). Literature related to depression and anxiety in the general population, as well as women, persons with HIV, African Americans, and pregnant women will be discussed in the following sections.

\section{Depression in the General Population}

Depressive disorders include a wide range of specific illnesses including major depressive disorder (MDD), dysthymia, and bipolar disorder (Barroso et al., 2008). Depression is considered a major health problem and is one of the leading causes of disability in the United States (R. C. Kessler et al., 2003; R. C. Kessler \& Kessler, 2003). Patients with depressive symptoms describe poor physical and social functioning, which limits their activity (K. B. Wells, Stewart et al., 1989). Impaired functioning has a direct impact on not only the patient, but also their families and communities.

An estimated 14.8 million adults or $6.7 \%$ of the U. S. population suffers from depression in any given year (R. C. Kessler et al., 2005; Wang et al., 2005). Healthy women in the general population are twice as likely to be diagnosed with depression as their male counterparts (Blazer, Kessler, McGonagle, \& Swartz, 1994; R. C. Kessler et al., 2003; Wang et al., 2005); depression is the leading cause of disability in women (R. C. Kessler, 2003). Despite these facts, research has shown that general practitioners often fail to diagnose depression in primary care practices (Goldberg \& Huxley, 1992; R. C. Kessler et al., 2003).

A wide range of literature focuses on depression and its prevalence in the general population (R. C. Kessler et al., 2005; R. C. Kessler, McGonagle, Zhao et al., 1994) with prevalence rates ranging from 10 to $21.3 \%$ (R. C. Kessler, McGonagle, Zhao et al., 1994; J. K. Myers et al., 1984; Olfson \& Pincus, 1996; Olfson et al., 2000). Significant depressive symptoms have been documented in primary care populations (K. B. Wells, Golding, \& Burnam, 1989), and depression is the most frequently studied outcome in populations with chronic diseases such as diabetes (Gore, Krupski, Kwan, Fink, \& Litwin, 2005; M. Gore et al., 2005; Ludman et al., 2004; McKellar, Humphreys, \& Piette, 2004), coronary artery disease (Rudisch \& Nemeroff, 2003; Ruo et al., 2003), cancer (C. H. Kroenke et al., 2005; Williams \& Dale, 2006) and HIV (Ciesla \& Roberts, 2001; LowBeer et al., 2000; Mock, Phillips, \& Sowell, 2002).

Literature illustrating racial differences in diagnoses of depression has been documented for decades (Neighbors, Jackson, Campbell, \& Williams, 1989), however disparity continues (Neighbors, Trierweiler, Ford, \& Muroff, 2003). Depression is 
diagnosed more frequently in White Americans than African Americans (Neighbors et al., 2003); schizophrenia is diagnosed more frequently in African Americans (Neighbors et al., 2003). The subjective nature of the clinical diagnosis of depression suggests that clinicians' bias may lead to inaccurate diagnoses despite the use of structured diagnostic criteria (Neighbors et al., 2003).

In one of the largest national epidemiologic studies conducted, Kessler and colleagues (R. C. Kessler, McGonagle, Zhao et al., 1994) examined prevalence rates of depression in a sample of 8098 persons from communities in 48 states. Participants' age ranged from 15 to 54 years. Data were collected by trained interviewers using the Composite International Diagnostic Interview (World Health Organization, 1990). Lifetime prevalence of major depression was estimated at $17.1 \%$. When the sample was assessed for current depression (within the last 30 days), the prevalence rate averaged $4.9 \%$. Distributions across age ranges were constant, but were twice as high in females as in males. The highest lifetime prevalence rates (21.1\%) were noted in African American women between the ages of 35 to 44 years. These findings emphasize the need to screen African American women for depression and justify the need for the current study as many of the participants in the current study were women within these age ranges. Additional results pertinent to the current study were: 1) persons in lower socioeconomic ranges were at higher risk for depression than their more affluent counterparts and 2) persons in urban areas were at greater risk rural participants. The current study was implemented in an urban area and most participants were poor.

Anxiety in the General Population

The American Psychiatric Association (APA) classifies anxiety disorders as many different, distinct disorders including: panic disorder, agoraphobia without panic disorder, specific phobia, social anxiety disorder, generalized anxiety disorder, posttraumatic stress disorder, obsessive-compulsive disorder, and separation anxiety disorder. The four most common anxiety disorders are panic disorder, social anxiety disorder, generalized anxiety disorder, and posttraumatic stress disorder (Gillock, Zayfert, Hegel, \& Ferguson, 2005; Issakidis, Sanderson, Corry, Andrews, \& Lapsley, 2004; Wittchen, 2002; Wittchen et al., 2002). Approximately 130 million people in the United States have a lifetime history of anxiety (Regier et al., 1988). In studies of clinical samples, $50 \%$ or more of patients with a primary anxiety disorder have at least one additional anxiety or mood disorder (Fiore et al., 2001). An estimated $25 \%$ of all primary care patients have an anxiety disorder (Sartorius, Ustun, Lecrubier, \& Wittchen, 1996). Despite its high prevalence, only $15 \%$ to $36 \%$ of patients with anxiety are diagnosed in primary care settings (D. Kessler, Lloyd, Lewis, \& Gray, 1999).

In one of the largest replicated epidemiologic studies conducted $(N=9282)$, Kessler and colleagues (R. C. Kessler et al., 2005) examined lifetime prevalence and age of onset of DSM-IV diagnoses. Face-to-face assessments were conducted by trained professional interviewers from the Institute for Social Research at the University of Michigan, Ann Arbor, between February 2001 and April 2003. The response rate was $70.9 \%$. The estimated lifetime prevalence of anxiety disorders in the general population 
was $28.8 \%$. Median age of onset for anxiety disorders was quite young (11 years) compared to depressive disorders (30 years).

In another epidemiologic study, patients from 15 primary care clinics in the U. S. completed self-report questionnaires and a follow-up telephone interview (K. Kroenke, Spitzer, Williams, Monahan, \& Lowe, 2007). Demographic characteristics of the sample included an average age of 47.1 years, majority female (69\% of the sample was women), and majority (81\%) non-Hispanic White Americans. The sample was educated, like the current study sample. Many of the participants (29\%) had a high school degree or equivalent and 39\% had some college. Of the 965 participants, $19.5 \%$ had at least one anxiety disorder (95\% CI, $17 \%$ to $22.1 \%)$. Almost half (41\%) of the participants diagnosed with an anxiety disorder were not receiving treatment.

Prevalence of anxiety symptoms among women is twice as high as those for men (Breslau, Schultz, \& Peterson, 1995; J. M. Murphy et al., 2004; Olfson et al., 2000) which informs the present study, as anxiety symptoms are not routinely and systematically assessed in populations of women. Recent literature related to anxiety includes investigation of genetic correlates for anxiety (Landgraf \& Wigger, 2003). Unlike depression, evidence does not support a significant association between family history of anxiety disorders and a personal risk for anxiety disorders (USDHHS, 1999). However, epidemiologic studies consistently document high rates of co-morbid depression and anxiety within the general population (Brown, Campbell, Lehman, Grisham, \& Mancill, 2001; R. C. Kessler et al., 2005; R. C. Kessler, McGonagle, Nelson et al., 1994; J. M. Murphy et al., 2004; Olfson et al., 2000). The next section will discuss co-morbid depression and anxiety.

\section{Co-morbid Depression and Anxiety in the General Population}

Overlapping symptoms of anxiety and depression often occur in persons diagnosed with mood disorders (Brown et al., 2001; R. C. Kessler et al., 2003; R. C. Kessler, McGonagle, Nelson et al., 1994; J. M. Murphy et al., 2004). The significant rates of co-morbidity have prompted investigation into overlapping symptoms, diagnostic trends, and effective treatment regimens (R. C. Kessler et al., 2005; R. C. Kessler, McGonagle, Nelson et al., 1994; Wang et al., 2005; Zimmerman \& Chelminski, 2003). Hirschfeld (Hirschfeld, 2001) recognized a lack of information regarding the co-existence of these disorders in primary care settings, and subsequent epidemiologic studies (R. C. Kessler et al., 2005) illustrate high levels of co-morbid conditions within both the general population and in primary care populations (Rodriguez et al., 2004). Because previous research indicates that the prevalence of depression and anxiety is higher in women than men (Blehar \& Oren, 1995; Breslau et al., 1995; J. M. Murphy et al., 2004; Olfson et al., 2000) and these conditions often co-exist, it reasonable to expect women to experience co-morbid depression and anxiety more often than men.

The need for routine screening is evident due to high prevalence rates and the fact that depression and anxiety are common in the context of physical illnesses such as cardiac disorders, hypertension, gastrointestinal disorders, genitourinary disorders, and 
migraines (M. C. Harter, Conway, \& Merikangas, 2003). High prevalence rates of comorbid depression and anxiety have been noted in several national studies (Brown et al., 2001; R. C. Kessler et al., 2005). Brown and colleagues (2001) reported high comorbidity rates in a sample $(\mathrm{N}=1,127)$ of patients with diagnosed anxiety and mood disorders who were seeking treatment at a university-based specialty clinic. Co-morbidity for specific anxiety and mood disorders were noted in patients with generalized anxiety disorder, with $65 \%$ having a current co-morbid mood disorder and $88 \%$ with a lifetime co-morbid anxiety or mood disorder. Ninety-two percent of patients with post-traumatic stress disorder had a current mood disorder and all had a lifetime history of a mood disorder. Kessler and colleagues (R. C. Kessler et al., 2005) reported co-morbidity rates for anxiety disorders, mood disorders, impulse control disorders, and substance use disorders in a national epidemiologic study. In this sample of 5692 participants, $55 \%$ had a single diagnosis, $22 \%$ had two diagnoses, and $23 \%$ had three or more diagnoses. The numbers and types of diagnoses have illustrated racial differences.

Mood Disorders in the African American Population

Researchers examining mental health diagnoses by race and ethnicity have cited racial differences in DSM diagnoses (Loring \& Powell, 1988; Neighbors et al., 2003). Schizophrenia is diagnosed more frequently in African Americans than White Americans, while White Americans are more likely to receive a diagnosis of bipolar disorder than African Americans (Neighbors et al., 2003). These differences in mental health diagnoses were studied by Neighbors and colleagues (Neighbors et al., 2003), through semi-structured diagnostic instruments administered to a group $(N=665)$ of African American and White American psychiatric inpatients. Results indicated that race was related to diagnosis and dependent upon accuracy of clinical judgment. Pre-existing cultural beliefs may impact clinical decision making. The authors recommended that cultural information be integrated into clinical training programs to decrease this bias.

Racial differences in access to mental health care have illustrated disparities that affect African Americans. African Americans have less access to health care across health care disciplines, including mental health and psychiatric services. Some reports indicate that African Americans are more likely to have depression than their White counterparts (Gazmararian, James, \& Lepkowski, 1995; Hough, Brumitt, \& Templin, 1999; Husaini et al., 1990; McBarnette, 1996); while other studies reveal that rates of major depression are either higher than (Blazer, Kessler, McGonagle, \& Swartz, 1994) or equivalent to (Berkman et al., 1986; Murrell, Himmelfarb, \& Wright, 1983) the rates among non-Hispanic whites than African Americans. Plant and Sachs-Ericsson (Plant \& Sachs-Ericsson, 2004) reported that ethnic minority women report more depressive symptoms than Caucasian women, while George and Lynch (George \& Lynch, 2003) reported that African Americans experience greater stress than White Americans and therefore greater depressive symptoms than White Americans. Lower income levels have been associated with greater stress levels due to the inability to meet basic needs, which could increase depressive symptoms (B. P. Dohrenwend \& Dohrenwend, 1969; Turner \& Lloyd, 1999). A plausible explanation for the difference in results of these studies was 
the large amount of missing income data in the Blazer and colleagues' (Blazer et al., 1994) study.

Teresi and colleagues (Teresi et al., 2002) explored racial differences in the recognition of depressive symptomatology. In a population of nursing home residents, $(N=270)$ African Americans had fewer depressive symptoms than other ethnic groups. The referral of patients to psychiatry may have had an influence on these results, as the authors noted that the persons with the most involvement with the patients (nursing assistants) gave the most accurate report of depressive symptomatology compared to other health care professionals (nurses, social workers) engaged in the care of the patients. One possible reason for this difference in perceptions could be related to race. Nursing assistants were more often African American, whereas nurses and social workers in the study were predominately Caucasian.

Economic differences have been investigated as possible barriers to mental health care among African Americans with conflicting results. Economic barriers such as unemployment, low income, or lack of health insurance are often used to explain racial disparities in health care among African Americans. However, research has shown that African Americans who had health insurance were actually less likely to use mental health services than White Americans (Snowden \& Thomas, 2000). Furthermore, African Americans have low overall rates of outpatient mental health service use (Alegria, Canino, Rios, \& et al., 2004; K. Wells, Klap, Koike, \& Sherbourne, 2001). Moreover, discrepancies in use of mental health services may be related to stigma related to mental illness within African American communities. Stigmatization of mental illness is one of the main reasons treatment is delayed until the disorder becomes debilitating or a crisis develops (United States Department of Health and Human Services, 1999; Zhang \& Snowden, 1999). Literature illustrates low rates of mental health care by African Americans. Although several reasons have been hypothesized, stigmatization remains a strong force that must be addressed. The current study examined anxiety and depressive symptoms among African American HIV-positive women, a population drenched in shame, stigma, and isolation.

\section{Depression and Anxiety in HIV-positive Patients}

Mood disorders are common among persons living with HIV in the United States (Bing et al., 2001; Ickovics et al., 2001; Moore et al., 1999; Morrison et al., 2002). Depressive symptoms are the most commonly examined psychiatric symptom in HIV populations. Early in the epidemic, homosexual men represented a large proportion of samples in HIV literature, which appropriately reflected the HIV population at that time (Atkinson et al., 1988; Bing et al., 2001; Ickovics et al., 2001; Leserman et al., 1999; Lyketsos et al., 1996; Perkins et al., 1994; Rabkin, Goetz et al., 1997). Recent literature describes the effects of mood disorders in HIV-positive women (Cruess et al., 2005; Evans et al., 2002; Ickovics et al., 2001; Morrison et al., 2002; Perkins et al., 1994; Rabkin, Goetz et al., 1997), as the HIV epidemic grew to impact demographically different populations across the country. 
Many of the published research studies regarding mood disorders in the HIV literature are cross-sectional, capturing information during one specific time point. HIV, like many chronic diseases, has stages and progresses differently based on multitude of factors. Cross-sectional studies may not accurately reflect the varying levels of depressive and anxiety symptoms that occur throughout the course of the illness. Dissimilar findings across studies may indicate differences within the sample, such as varying stages of disease and varying lengths of HIV diagnosis which likely influences the intensity of depressive symptoms.

HIV literature reveals varying rates of co-morbid depression and anxiety, however similar trends are noted. Rates of mood disorders in a sample of HIV-positive women $(n=93)$ were $19.4 \%$ for MDD, $4.3 \%$ for dysthymic disorder, $2.2 \%$ for generalized anxiety disorder, and $10.8 \%$ for any anxiety disorder (Morrison et al., 2002). Higher rates of depression and anxiety have been reported in populations of HIV-positive men $(n=56)$, with rates as high as $30.3 \%$ for MDD and $39.3 \%$ for generalized anxiety disorder (Atkinson et al., 1988). In general, higher rates of depressive disorders (MDD) have been illustrated in HIV-positive groups, when compared to their HIV-negative counterparts. Maj and colleagues (Maj et al., 1994) reported MDD rates of $7.14 \%$ in a group of $602 \mathrm{HIV}$-positive men and women, compared to a lower rate of MDD $(1.98 \%)$ in the group of HIV-negative men and women $(n=353)$. Contrasting results were seen in Rabkin and colleagues'(Rabkin, Goetz et al., 1997) study, with lower rates of MDD seen in HIV-positive men $(n=183)$ than HIV-negative $(n=65)$ men $(7.65 \%$ vs. $8.33 \%$, respectively). However, in this group of men, higher rates of dysthymic disorder $(13.11 \%)$ were seen in the group of HIV-positive men than the group of HIV-negative men $(4.76 \%)$, indicating the need for comprehensive assessment of a wider range of mood disorders.

The prevalence of a wider range of psychiatric disorders among HIV-positive men and women was assessed in a large $(N=2864)$ epidemiologic study (Bing et al., 2001). Trained interviewers assessed major depression, dysthymia, generalized anxiety disorders, and panic attacks. Results illustrated that almost half of the participants had scores indicating presence of a psychiatric disorder. The most common disorders included MDD (36\%) and dysthymia (26.5\%) with lower rates noted for generalized anxiety disorder (15.8\%) and panic attacks (10.5\%). The high numbers of HIV-related side effects in the sample may explain the higher rates of depressive disorders and dysthymia in the study. Anxiety disorders were lower in this sample than seen in the general population. A plausible explanation for this finding may be the high frequency of marijuana use that was reported by the participants.

Previous studies provide consistent evidence that HIV-positive persons experience significant levels of depression, yet the concept of depression has been used broadly, with some studies focusing on diagnoses of specific mood disorders such as MDD (Bing et al., 2001), while others focused on the presence of depressive symptoms (Atkinson et al., 1988; Bing et al., 2001; Lipsitz et al., 1994; Perkins et al., 1994; Stern et al., 1992). In some cases, the effects of mood disorders on HIV treatment are specified, such as adherence to HAART or medical appointments (Starace et al., 2002), while other 
research has focused on the detrimental effects that depression has on HIV disease progression (Cruess et al., 2003; Leserman, 2003), morbidity, and mortality (Ickovics et al., 2001).

Thus, evidence suggests that when compared to their HIV-negative counterparts, HIV-positive persons may experience greater levels of depressive and anxiety symptoms. Studies must be viewed with caution, as prevalence rates vary greatly among studies. Rates of depressive symptoms alone have ranged from 5\% to 50\% among HIV-positive persons (Atkinson et al., 1988; Bing et al., 2001; Leserman, 2003; Lipsitz et al., 1994; Perkins et al., 1994; Stern et al., 1992). The wide range of assessment techniques, varied instrumentation, varied outcome measurements (depressive symptoms, anxiety symptoms, MDD, dysthymia, generalized anxiety disorder), combined with differences among samples (concomitant substance use and differences in HIV disease progression) across studies makes comparison of findings difficult; however, similar trends are noted. Because most studies in the HIV literature do not include an assessment of multiple risk factors or vulnerabilities for mood disorders, this lack of consensus in findings is not unexpected.

\section{Depression and Anxiety in African American HIV-positive Women}

Women of color are now one of the fastest growing groups of newly infected persons with HIV (Centers for Disease Control and Prevention, 2002, 2005, 2007a; Ickovics et al., 2001; Morrison et al., 2002). Consistent with the general HIV literature, varying prevalence rates of mood disorders are documented in women who are HIVpositive. Prevalence rates of mood disorders vary from $30 \%$ to $60 \%$ among populations of women who are HIV-positive (Ickovics et al., 2001; Morrison et al., 2002; D. K. Smith et al., 1996; Taylor, Amodei, \& Mangos, 1996). African American women, although underrepresented in many other areas of research, were well represented in several HIV studies. In Ickovics' (Ickovics et al., 2001) study, 62\% of the women in the sample were African American, while Morrison's study contained approximately 55\% African American women. Depressive symptoms are the most common mental health outcome assessed in studies of HIV-positive women.

Consistent with earlier HIV literature that compared HIV-positive men with HIVnegative men higher rates of depressive symptoms have been documented in similar populations of women. Ickovics and colleagues (Ickovics et al., 2001) examined the prevalence of depressive symptoms among HIV-positive women $(N=765)$ in a prospective longitudinal cohort study. The majority of the sample (62\%) was African American, unemployed (84\%), and denied substance use during the study duration (54\%). Participants were evaluated every six months and required at least three study visits for analysis. Depressive symptoms were measured using the CES-D (Radloff, 1977), which captured self-reported symptoms experienced within the past seven days. Chronic depressive symptoms were defined as elevated CES-D scores (using the cut-off score of 16 or higher) for $75 \%$ of their study visits, intermittent depressive symptoms were defined as elevated CES-D scores for $26-74 \%$ of their study visits, and limited or no depressive symptoms were classified as no more than $25 \%$ of their study visits revealing 
CES-D scores at or above 16 . Almost half of the sample (42\%) reported chronic depressive symptoms, while $35 \%$ reported intermittent depressive symptoms.

Morrison and colleagues (Morrison et al., 2002) examined differences in mood disorders, including depressive and anxiety symptoms, among HIV-positive and HIVnegative women in a large, longitudinal cohort study $(N=155)$. Although the authors did not specifically document a case-control design, one is implied. The authors do not indicate if participating women were matched according to race, age, or any other demographic characteristic. There was a larger group of HIV-positive women $(n=93)$ than HIV-negative women $(n=62)$. Racial distributions were similar in both groups, with the majority of the sample being African American (55.9\% in HIV-positive group; 53.2\% in the HIV-negative group).

Comprehensive measurements included physical and psychological exams, interviews, and the completion of questionnaires [Hamilton Depression Rating Scale [(M. Hamilton, 1967)] and Hamilton Anxiety Rating Scale [(M. Hamilton, 1959)]. The cohort design allowed the researchers to follow populations over time and gather information about the natural history of disease, examine sequences and patterns over time as well as inferring causality, which may illustrate risk factors. Two groups of women were followed from 1997-2000. Differences in the rates of depression and anxiety disorders in the group of HIV-positive women were compared to a group of HIV-negative women.

Results indicated that HIV-positive women had higher prevalence rates of major depressive disorder (19.4\%) than HIV-negative women $(4.8 \%)$ and these differences were statistically significant $(\mathrm{t}=-4.9, \mathrm{df}=152, p<0.0001)$. Similar results were noted for diagnoses of anxiety disorders, with $10.8 \%$ of the HIV-positive women being diagnosed with any anxiety disorder and $2.2 \%$ of the HIV-positive women being diagnosed with generalized anxiety disorder. Lower rates of any anxiety disorder were noted in the HIV-negative group of women $(6.5 \%)$, but the HIV-negative women had higher rates of generalized anxiety disorder (3.2\%) than their HIV-positive counterparts $(2.2 \%)$. Anxiety symptom scores were higher in the group of HIV-positive women when compared to the HIV-negative group, and these differences were statistically significant $(\mathrm{t}=-4.5, \mathrm{df}=152, p<0.0001)$. HIV-positive women $(n=47 ; 50.5 \%)$ also had higher rates of active substance use than their HIV-negative counterparts $(n=19 ; 30.6 \%)$, a difference that was statistically significant (odds ratio $=2.3,95 \% \mathrm{CI}=1.2-4.6 ; p=0.02$, Fisher's exact test)

Demographic characteristics of women who are HIV-positive include multiple complex factors which may influence the risk for mood disorders. Caregiving roles as mothers and partners may add to stress and distress that is experienced by this population (Fiore et al., 2001). Literature reveals that HIV-positive women report high levels of fatigue and anxiety (Barroso, 2002; Barroso, Carlson, \& Meynell, 2003; Barroso et al., 2008; Meynell \& Barroso, 2005) are frequent victims of violence (Leibschultz, Feinman, Sullivan, Stein, \& Samet, 2000), and live in poverty (Ickovics et al., 2001; Wohl et al., 1998). 
African American women with HIV are a unique population with additional stressors that may contribute to depressive and anxiety symptoms. Understanding the psychological burden the diagnosis of HIV brings to patients is imperative to appropriately counsel and guide them through the course of this chronic disease. Additional research regarding possible vulnerabilities to depressive and anxiety symptoms is necessary.

\section{Depression and Anxiety in Pregnancy}

In studies focused on mood disorders and pregnancy, the most frequently studied mood disorder is postpartum depression. However, results from investigations designed to examine predictors of postpartum depression consistently include antenatal depression as one of the main risk factors for postpartum depression. Therefore, the assessment of mood disorders in pregnancy remains a critical aspect of comprehensive prenatal care. In the next section the correlates of mood disorders and pregnancy will be discussed.

Depression is the leading cause of disability in women (R. C. Kessler, 2003) and often first appears following pregnancy. Fifty to seventy percent of women experience psychological changes following childbirth (Mosack \& Shore, 2003). The overall psychological reactions commonly known as the "baby blues" include transient, mild states of tearfulness, anxiety, restlessness, and irritability. Due to the high frequency of occurrence, these symptoms are often considered a normal part of the postpartum period, appearing as early as the first postpartum day. The duration of these symptoms is short, lasting approximately ten days in most affected women. Symptoms may reappear after exposure to decreased sleep quality and duration that are often associated with neonatal care. Increase in dysmorphic syndrome disorders is often attributed to somatic changes, not hormone level changes that occur immediately after childbirth (Dixit \& Crum, 2000; Holahan, Moos, Holahan, Cronkite, \& Randall, 2003; Nott, Franklin, Armitage, \& Gelder, 1976; Schutte, Moos, \& Brennan, 1995; Swain, O'Hara, Starr, \& Gorman, 1997)

Over the past few decades, an increasing number of studies have led to the identification of multiple variables that are correlated with post-partum depression. In contrast to earlier theories that included dramatic hormone changes that occur during pregnancy and immediately after delivery, the current etiology of mood disorders in pregnancy suggests a multifactorial process (Casswell, Pledger, \& Hooper, 2003; Neighbors et al., 2003; L. E. Ross, Gilbert Evans, Sellers, \& Romach, 2003). Investigators have examined specific risk factors of post partum depression and found that the most prevalent are: antenatal depression and anxiety, personal and family history of depression, life stress, and lack of social support (C. T. Beck, 2001; P. Cooper \& Murray, 1997; P. J. Cooper \& Murray, 1998; Howell, Mora, \& Leventhal, 2006; O'Hara \& Gorman, 2004).

Early identification of vulnerable populations of women is essential. Prevalence rates of postpartum depression vary widely; however research suggests that 10 to 50 percent of women experience postnatal depression. Varying rates are due to sampling techniques, populations studied, and the wide variety of screening assessments used 
across studies. Postpartum depression is the most common disorder that affects women following childbirth (C. T. Beck, 1998; Cox et al., 1987). Researchers have illustrated that levels of depressive symptoms in the immediate postpartum period are significant predictors of postpartum depression (Dennis, 2004; Hannah, Adams, Lee, Glover, \& Sandler, 1992).

In a longitudinal study, depressive symptoms of women $(N=217)$ that delivered infants at two British hospitals were assessed at five days postpartum, and again at six weeks postpartum using the EPDS (Hannah, Adams, Lee, Glover, \& Sandler, 1992). Results indicated that women with EPDS scores greater than nine at five days postpartum were eight times more likely to have EPDS scores above nine, which is indicative of mild depressive symptoms, at six weeks postpartum. Similar results were found in Dennis' 2004 study of Canadian mothers $(N=594)$ where women were screened with the EPDS at one, four, and eight weeks postpartum. Elevated EPDS scores at one week postpartum were associated elevated EPDS scores at both four and eight weeks postpartum (Dennis, 2004). Racial descriptions of the Canadian participants were not provided, limiting comparisons to the current study of African American women.

Screening evaluations for post partum depression were evaluated in another study of postpartum women in North Carolina $(N=391)$. The majority of the sample $(84 \%)$ was Caucasian and had at least a high school education (74\%). Medical residents compared screening with the use of the Edinburg Postnatal Depression Scale with routine interviews of women that had delivered infants (Evins, Theofrastous, \& Galvin, 2000). Chi square analysis compared 79 women evaluated with the EPDS with 96 women evaluated with clinical evaluations (structured interviews). Women were assigned to one of the two screening measurements by delivery date. Results revealed the incidence of postpartum depression detection with the Edinburgh Postnatal Depression Scale was significantly higher than the incidence of spontaneous detection during routine clinical evaluation $(35.4 \%$ and $6.3 \%$, respectively; $p=.001)$. Investigators recommended that the EPDS is an effective method for screening postpartum women for depressive symptoms. The authors also recommend the EPDS as a useful instrument that should be introduced to medical residents during their training and used in their post resident practice. Thus, the evidence suggests that women with depressive symptoms immediately postpartum continue to experience depressive symptoms weeks after delivery, suggesting the need for early screening, identification and intervention. These results were important to the current study, and emphasize the need to not only screen women for depressive symptoms, but also train healthcare providers to implement early consistent screening in their practices.

Mood disorders in pregnancy are a complex phenomenon which can have devastating consequences on both the mother and the infant. Complex psychological changes may occur in women during and after pregnancy, and may vary in duration, intensity, and severity. One third of women that experience postpartum depression, but are either untreated or undiagnosed, may continue to suffer from depression for one year. Therefore, it is important that women be screened for signs and symptoms of depression 
as early as possible. Many women do not report symptoms of postpartum depression (C. T. Beck \& Gable, 2000), fearing social stigma (C. T. Beck, 2001).

In studies focused on postpartum depression, investigators have examined the relationship between postpartum depression and major depression. The strongest predictor of postpartum depression is the presence of prenatal depression (C. T. Beck, 1996; O'Hara \& Gorman, 2004). Despite the grim realization of the effects of postpartum depression and the prevalence of the condition, many women are not routinely screened. Recognizing signs and symptoms of depression is imperative. Screening instruments have been shown to be useful in identifying depression by identifying depressive symptomatology. Use of such questionnaires may help identify women that experience signs and symptoms of depression. The most widely used instrument is the Edinburgh Postnatal Depression Screen (EPDS) (Cox et al., 1987), which was one of the instruments used to measure depressive symptoms in the current study. Assessment of underlying vulnerabilities for depression and anxiety may enhance screening procedures; inherited and acquired vulnerabilities for these disorders will be discussed in the next section.

\section{INHERITED VULNERABILITIES FOR MOOD DISORDERS}

Human genetic makeup is determined by building blocks that are provided by biologic family. The term inherit can be defined as to receive from one's ancestors (Mish, 1997). Vulnerability reflects susceptibility to wounds, or capability of being wounded (Mish, 1997). Inherited vulnerabilities are often the initial influences that affect not only the early life experiences, but also lay the groundwork for future coping strategies and lifestyle choices. In the current study, the term vulnerability referred to susceptibility to mood disorders; both inherited and acquired vulnerabilities were examined. The current study evaluated two inherited vulnerabilities that influence the risk for developing mood disorders: family history of mood disorders and family history of alcohol and/or drug use.

\section{Family History of Mood Disorders}

Family history of mood disorders has long been acknowledged as a strong predictor of mood disorders in many patients (Gershon et al., 1982; Klein, Lewinsohn, Seeley, \& Rohde, 2001; Weissman et al., 1987). Depressive symptoms are the most frequently examined mood disorder in family history research. The method by which family history information is ascertained varies across studies and ranges from a report of a family member with a mental health history to structured interviews and psychological assessment of family members to clinicians determining the presence or absence of psychiatric diagnoses. The later is much more costly and labor intensive but may be more accurate. The family history method has been shown to have good sensitivity in family studies of depression (Kosten, Anton, \& Rounsaville, 1992; Zimmerman \& Chelminski, 2003) but less specificity. Conclusions about the relationship between family history of mood disorders and individual presence of mood disorders may be equivocal because most studies included self-report measures of family history. Despite acknowledged limitations of subjective reports, in the current study, participants reported family history 
of mood disorders on the Demographic Data Instrument. Using this approach, however, facilitates comparisons between the findings of the current study and findings of published studies that determine family history using a similar approach.

Numerous studies have consistently shown that the children born to parents with major depressive disorder have a three-fold risk of experiencing major depressive disorder in their lifetime (Downey \& Coyne, 1990; Kosten et al., 1992; Lieb, Isensee, Hofler, Pfister, \& Wittchen, 2002; Weissman et al., 1984; Williamson, Birmaher, Axelson, Ryan, \& Dahl, 2004; Zimmerman \& Chelminski, 2003). Lieb and colleagues (Lieb et al., 2002) conducted an analysis of demographic, structured interview, and questionnaire data obtained as part of a prospective longitudinal study $(N=2427)$ designed to explore the incidence, prevalence, and risk factors associated with mental disorders. Subjects were recruited from a community sample in Munich Germany; racial descriptions of the sample were not provided. Data were collected from adult participants and their children at entry and then four years later. Most participants were mothers $(n=1026)$ although some participants were fathers $(n=27)$ if the mother was not available. Diagnoses of depression, anxiety, and substance use disorders were made based on DSM-IV guidelines. Results indicated that major depression in a parent, either the mother or the father, increased the risk for mental disorders in their male and female children. Children's diagnoses revealed that children born to two parents with depression were at no greater risk for developing mental disorders than children born to one parent with depression. However, onset of symptoms differed in children with one or both affected parents, with earlier onset of depression noted in children with two affected parents. Severity of depression among children (defined as the number of depressive episodes and level of treatment seeking) was greater if a parent had a history of depression. A limitation of the study was the assessment of only one parent. Interviews were used to gather additional information regarding the nonparticipating parent and most of the parents assessed were mothers. These aspects of methodology may have compromised the results, as comprehensive assessments of both parents might yield greater insights regarding the influence of this risk factor for mood disorders.

Much of the published literature regarding family history for mood disorders focuses on parental histories or two specific generations of family members (Downey \& Coyne, 1990; Kosten et al., 1992; Lieb et al., 2002; Weissman et al., 1984; Williamson et al., 2004; Zimmerman \& Chelminski, 2003) however, multigenerational studies are now being conducted to further examine the strength of familial history as a risk factor. A three generation longitudinal study was conducted by Weissman and colleagues (Weissman et al., 2005). The twenty year study included a sample $(N=161)$ of grandchildren, their parents and grandparents. The third generation of the original cohort study was the focus of this study. The original cohort study reported a total sample of 73 adolescents with MDD and 37 adolescents without MDD. The majority of the sample was Caucasian (58.9\% in sample with MDD; 59.4\% without MDD). African Americans represented $9.6 \%$ of the sample with MDD and $21.6 \%$ of the sample without MDD. Clinicians blinded to family history data collected information from participants, specifically lifetime history of diagnosis of a psychiatric disorder. This method of data collection allowed a less biased approach to data collection. . High rates of psychiatric 
disorders were noted in the grandchildren with a two generation history of major depression. Subjects with depressed grandparents also had increased risk of anxiety (relative risk, 5.17; 95\% confidence interval, 1.4-18.7; $p=.01$ ). Subjects with depressed parents had an increased risk of any psychiatric disorder (relative risk, 5.52; 95\% confidence interval, $2.0-15.4 ; p=.002$ ). Severity of impairment was measured with multiple instruments, diagnostic interviews, and presence of divorce in the first and second generations. Results indicated that the more impaired the depressed parent, the higher the rate of mood disorders in the grandchildren (relative risk, 2.44; 95\% confidence interval, 1.1-5.5; $p=.03$ ). Results also indicated an association between parental MDD and child diagnosis of MDD, which varied based upon the presence of grandparental MDD status. Grandchildren whose parents and grandparents illustrated moderately to severely impairing depression exhibited the highest rates of psychopathology, indicating the need for multi-generational historical information.

Family psychiatric history has been shown to play an important part in adolescent psychiatric assessment, furthering the notion that markers or vulnerabilities may be present early in the developmental process. King and colleagues (King et al., 2006) investigated the family psychiatric histories of a group of adolescents $(N=71)$ that were being treated for MDD. Adolescents and their parents completed interviews and questionnaires to assess the presence or absence of severe behavioral disinhibition. The adolescents with increased behavioral disinhibition had a higher prevalence of drug use disorders in their biological fathers, higher proportion of paternal second degree relatives with alcohol use disorders, drug use disorders, and (King et al., 2006) psychiatric hospitalizations. The adolescents with increased behavioral disinhibition also had a higher prevalence of maternal second degree relatives with antisocial personality disorder. Results from this study, as well as other psychiatric studies in children and adolescents, encourage early screening and assessment of children with inherited vulnerabilities for mood disorders. The current study examines the impact of inherited vulnerabilities on depressive and anxiety symptoms.

Prevalence of family history of depression was examined in an exclusively female community sample by Sullivan and colleagues (Sullivan et al., 1996). The authors hypothesized that a family history of depression increased the likelihood that a person would seek treatment for mental health evaluation; therefore they examined the prevalence of depression within a community sample.

Family history of depression was assessed in three groups of women: (1) 54 women randomly selected from the community with depression in the prior year, (2) 41 women who entered a clinical trial for depression, and (3) 37 randomly selected women from the community who had no history of depression in the prior year. Presence or absence of depression in the parents and siblings of the women with depression was assessed by the family history method. Depressed women had higher family history scores of depression than other two groups ( $p<0.00005$ for both groups). As with many family history studies, direct interviews with relatives were not completed. Therefore the impact of differential reporting may have affected the study results. 
Although much of the family history literature focuses on genetic vulnerabilities, research suggests that the impact of familial mental disorders can be explained through both genetic and environmental factors (Beardslee, Versage, \& Gladstone, 1998; Rutter, Silberg, O'Connor, \& Simonoff, 1999a, 1999b). The family history component of mental disorders suggests a familial transmission of depression, with a possible category of "familial subtype" of depression (Weissman, Warner, Wickamaratne, Moreau, \& Olfson, 1997). However, both genetic and environmental factors influence depressive symptoms in both children and their parents, and investigators have recently incorporated this knowledge into their design (Sagrestano, Paikoff, Holmbeck, \& Fendrich, 2003).

Sagrestano and colleagues (Sagrestano et al., 2003) conducted a longitudinal study of child and parent depression in an inner city, urban, low-income population $(N=$ 302). All children were African American adolescents, aged 9-15. Two time points illustrated $7.3 \%$ of the parents and $3 \%$ of the children had depressive symptoms at the first collection, with $5.4 \%$ of the parents and $2.8 \%$ of the children were clinically depressed. Regression analysis revealed increases in conflict and decreases in parental monitoring were associated with higher depressive symptomatology in the children. Increases in conflict and decreases of positive parenting resulted in higher depressive symptomatology in the adults. The longitudinal design was a strength of this study. The current study was cross-sectional in nature, capturing data from participants at one timepoint, rather than multiple assessments over time.

No direct cause of mood disorders can be identified, although published literature suggests that both genetic and environmental factors contribute to mood disorders. Researchers note the importance of obtaining accurate histories regarding mood disorders, especially parental diagnoses, as well as information related to family interaction patterns, an environmental aspect of family history (Shiner \& Marmorstein, 1998). The varying degrees of severity, co-morbidity occurrences, recurrence rates, environmental factors, and treatment modalities make causal inferences difficult. Therefore, additional research, particularly longitudinal multi-generational research studies are needed. Environmental factors, such as family history of alcohol and/or drug use, have also been examined in psychiatric literature, and will be discussed in the next section.

Family History of Alcohol and/or Drug Use

Much of the previous literature regarding family history of alcohol and/or drug use and has focused on the relationship between family history of alcohol and/or drug use and the presence of individual mood disorders (Beardslee et al., 1998; Dixit \& Crum, 2000; Maier, Lichtermann, \& Minges, 1994; Todd, Geller, Neuman, Fox, \& Hickok, 1996). Alcohol abuse disorder is considered a mood disorder; familial transmission of alcohol dependence has been demonstrated (Reich, Cloninger, Van Eerdwegh, Rice, \& Mullaney, 1988; Reinherz, Paradis, Giaconia, Stashwick, \& Fitzmaurice, 2003). When compared to rates of alcoholism in the general population, increased rates of alcoholism are present in persons with a family history of alcoholism. The following epidemiologic study illustrates this finding. 
Rice and colleagues (Rice et al., 2003) examined the relative risk of alcohol dependence in parents and siblings of alcohol dependent subjects $(N=4099)$ in comparison with control subjects and their families $(n=1054)$. Survival analysis, using Kaplan-Meier and Cox proportional hazards model, were calculated based on the age at the onset of alcohol dependence. DSM-III-R criteria were used as the definition of alcohol dependence. Men had higher rates than women; relatives of alcoholics had higher rates than controls. Blacks (non-Hispanic) had lower relative risks than whites. Lower levels of education had higher relative risks ( $\mathrm{RR}, 2.83 ; p<0.0001)$ rates than higher levels of education. Interestingly, a father's alcohol dependence was a significant predictor $(p<0.0001)$ of alcohol dependence, whereas, a mother's alcohol dependence was not $(p=0.3)$. These results indicate that not only should family history of substance use be assessed, but what specific relatives and what ages the dependence began are also important aspects of a thorough history.

Much of the published literature regarding the relationship between family history of alcohol and/or drug use and mood disorders has shown a positive relationship (Dixit \& Crum, 2000; Winokur \& Coryell, 1991). In a sample of 1383 women in Baltimore, women with family histories of alcohol and/or drug use were more likely to be depressed, and more likely to report a lifetime history of another psychiatric disorder (Dixit \& Crum, 2000). Higher rates of family history of alcohol and/or drug use were seen in depressed women, but not in depressed men (Winokur \& Coryell, 1991). Child or adolescent onset of depression (MDD) has led investigators to examine risk factors for depression, and familial history of both alcoholism and mood disorders remain strong correlates. Todd and colleagues examined prevalence rates of alcoholism in relatives of children with depression and bipolar disorder (Todd et al., 1996). The impact of family history of alcohol and/or drug use is evident early; 39 of the 48 children or adolescents $(81.25 \%)$ with depression also had histories of alcoholism in either first or second degree relatives (Todd et al., 1996). Rates were higher among children with bipolar disorder (41 of 47 or $87.23 \%$ ) Although the prevalence rates of alcoholism have been high in samples of depressed persons across many ages, these statistics do not imply causation, as research objectives and methodology vary across studies.

Inherited vulnerabilities have been associated with mood disorders for decades (Dixit \& Crum, 2000; Downey \& Coyne, 1990; Lieb et al., 2002; Maier et al., 1994; Todd et al., 1996; Weissman et al., 1984; Williamson et al., 2004). Family history of mood disorders and family history of alcohol and/or drug use have been the most common genetic vulnerabilities for mood disorders. Although much of the published literature focuses on the relationship between family history and depression, associations have also been illustrated with other mood disorders, including bipolar disorder and schizophrenia. The current study examined family history of both mood disorders and alcohol and/or drug use in this population of African American women who were HIVpositive. Individuals are unique and have multi-faceted backgrounds, which provide justification for the comprehensive assessment of risk factors for mood disorders, including both inherited and acquired vulnerabilities. The acquired vulnerabilities for mood disorders will be discussed in the next section. 


\section{ACQUIRED VULNERABILITIES FOR MOOD DISORDERS}

The etiology of mood disorders has been viewed as both inherited and acquired. The ways of conceptualizing vulnerabilities to mood disorders are many and vary across the literature. The relationship between some acquired vulnerabilities such as alcohol and/or drug use, and mood disorders have been investigated for decades (Hesselbrock, Meyer, \& Keener, 1985; Holahan et al., 2003; Schutte et al., 1995) while others, such as sleep quality are more recent explorations (Kripke et al., 2004; Lee, Portillo, \& Miramontes, 2001). The current study focuses on five acquired vulnerabilities: alcohol and/or drug use, socioeconomic level, educational level, disclosure of HIV status, and history of sexual abuse or assault. These individual acquired vulnerabilities will be discussed in the next sections.

\section{Alcohol and/or Drug Use and Mood Disorders}

Studies examining the relationship between alcohol and/or drug use and mood disorders were fairly consistent. Previous research indicates that alcohol and drug use has been positively correlated with mood disorders Merikangas and colleagues (Merikangas et al., 1998) report that alcohol and drug use is often related to emotional trauma, psychological stress, and other mental health problems, with the substance being used as self medication in order to relieve emotional pain. Results of a large epidemiologic study (The Epidemiologic Catchment Area Study) indicated that approximately 30\% of alcohol and/or drug users in the United States had a psychiatric disorder (Regier et al., 1990).

Many studies have described the relationship between alcohol use and mood disorders (Dixit \& Crum, 2000; Holahan et al., 2003; Schutte et al., 1995). Differentiation by gender is noted in patterns of alcohol use and rates of depression, with women reporting heavier alcohol usage than men (Dixit \& Crum, 2000; Holahan et al., 2003; Schutte et al., 1995) and minorities, especially African Americans and Hispanics, reporting higher rates of alcohol use than White Americans. Literature reveals disparities in mental health care, particularly among African Americans and Hispanic Americans, when compared to White Americans (K. Wells et al., 2001). Lack of mental health and drug treatment services, decreased access to mental health care, and evaluation of quality of mental health care all issues that need to be addressed in order to alleviate racial and ethnic disparities (K. Wells et al., 2001).

A review of the literature reveals equivocal results regarding the timing of depressive symptoms and the onset of heavy drinking. Helzer and Pryzbeck (Helzer \& Pryzbeck, 1988) report depression preceding alcoholism in women; but alcoholism preceding depression in men. Similar findings were reported in a group of alcoholic women by Hesselbrock and colleagues (Hesselbrock et al., 1985), with depression preceding alcoholism. Contrasting results were reported by Wilsnack and colleagues (Wilsnack, Klassen, \& Wilsnack, 1986), with depression preceding heavy alcohol consumption in women. Plausible explanations for these different findings could be related to the cycle of self-medicating depressive symptoms, which may lead to depression related to heavy alcohol consumption (Dixit \& Crum, 2000). Literature also 
reveals that alcohol abuse has also been identified as a risk factor for suicide (G. Murphy, 2000). Higher rates of attempted and completed suicides have been noted in alcohol abusers than non-alcohol abusers (D. Lester, 2000).

HIV literature reflects high prevalence rates of depressive symptoms in intravenous drug users with HIV (Turrina et al., 2001). The sample included two groups of intravenous drug users, one HIV-positive, and the other HIV-negative. A group of HIV-positive persons were recruited from an outpatient infectious disease clinic in Italy. HIV negative persons were recruited from an outpatient methadone clinic. Major depressive disorder was diagnosed using DSM-IIIR criteria. Depressive symptom levels were higher in the group of HIV-positive persons $(36.2 \%)$ when compared to their HIVnegative counterparts (15.7\%). Specifically, $36.2 \%$ of the HIV-positive sample had a diagnosis of major depressive disorder, compared to $15.7 \%$ in the HIV-negative group. Similar findings were found for dysthymic disorder ( $7.1 \%$ and $3.9 \%$, respectively).

Alcohol and/or drug use is one of many acquired vulnerabilities associated with mood disorders. Socioeconomic status is another acquired vulnerability linked to mood disorders, and will be discussed in the next section.

\section{Socioeconomic Status}

Most of the studies published over the past several decades that have investigated income level and mood disorders have used varied measures of symptoms and diagnostic criteria for mood disorders. Although many different symptoms and diagnoses have been explored, depressive symptoms have been the most frequently examined mood disorder. Socioeconomic status (SES) has been defined by several indicators, including income, occupation, and education (Daly, Duncan, McDonough, \& Williams, 2002). The collection of information regarding SES provides information about an individual's access to social and economic resources (Daly et al., 2002). Researchers have found ambiguous findings related to SES and depressive symptoms. For women, education is the strongest of the SES predictors of depression (R. C. Kessler, 1982). For men, family income is more important (R. C. Kessler, 1982). Of the three SES measures, education and income are more important than occupational status for predicting distress (R. C. Kessler, 1982). The current study defined socioeconomic status as annual household income. Educational level and employment information were also captured on the Demographic Data Instrument.

Populations of economically disadvantaged persons have higher rates of mood disorders than their more affluent counterparts (B. P. Dohrenwend et al., 1992; Olfson et al., 2000; Reiger, Farmer, \& Rae, 1993). Inverse relationships between depressive symptoms and income levels have been documented consistently in the literature, with lower levels of income being associated with higher levels of depression (B. P. Dohrenwend et al., 1992; Link, Lennon, \& Dohrenwend, 1993)

Anxiety symptoms display a similar pattern (Mirowsky \& Ross, 1989) with inverse relationships illustrated in the literature. Individuals with higher income levels, 
tend to have lower levels of anxiety symptoms and distress (C. E. Ross \& Mirowsky, 1989). Persons with lower incomes are less likely to seek mental health treatment and often lack resources to access specialized mental health care, instead seeking mental health treatment from their primary care provider

Poverty among HIV-positive women has been demonstrated (Bunting, Bevier, \& Baker, 1999; Hudson, Kirksey, \& Holzemer, 2004). Literature suggests that poverty makes women at risk for a multitude of problems, including poor access to health care. Poverty in HIV-positive women has been associated with worrying about daily survival, fears of domestic violence, poor self-esteem, and lack of health insurance, which leads to difficulty accessing health care. Poverty and lack of insurance has been described as one of the strongest factors related to the health care disparities among African Americans (Mayberry, Mili, \& Ofili, 2000). Previous studies have found that patients who are women, members of racial and ethnic minorities, poorer, less educated, or uninsured are less likely to receive needed care, due to lack of access to care (Ayanian, 1994; Kahn et al., 1994; Smedley, 2006). Educational level will be discussed in the next section as another acquired vulnerability for mood disorders.

\section{Educational Level and Mood Disorders}

Most of the literature published regarding educational level and mood disorders has been consistent over time (Morrison et al., 2002; Wang et al., 2005). The interwoven relationship between educational level and income is demonstrated in one's access and use of mental health services. Higher levels of education have been associated with higher levels of income. Consequently, higher levels of income offer financial resources to pay for mental health services. Persons with high levels of income are more likely to have health insurance. Educated consumers are more likely to access mental health services (Wang et al., 2005) and may be have greater knowledge regarding pharmaceutical treatment of mood disorders (K. B. Wells, Stewart et al., 1989).

Morrison and colleagues (Morrison et al., 2002) illustrated the relationship between educational levels and depressive symptoms in a sample of women $(N=155)$. Depressive symptoms were measured in both HIV-positive $(n=93 ; 55.9 \%$ African American) and HIV-negative ( $n=62 ; 53.2 \%$ African American) women with the Hamilton Depression Scale (M. Hamilton, 1967). Higher depressive symptom scores were associated with less education in both groups of women, and these differences were statistically significant $(\mathrm{t}=2.1$, df $152, \mathrm{p}=0.04$; beta $=-0.6,95 \% \mathrm{CI}=-1.2$ to -0.02$)$. Although educational level is an acquired vulnerability that has not been extensively studied in HIV-positive women, the process of disclosure of one's HIV status is a concept that has been vastly explored in the HIV literature, and will be discussed in the next section. 


\section{Disclosure of HIV Status}

Disclosure of one's HIV status is a complex process, unique to that individual. Therefore, disclosure is not an isolated event, but rather a series of events or processes that are associated with both positive and negative outcomes (Kalichman, DiMarco, Austin, Luke, \& DiFonzo, 2003; Vanable, Carey, Blair, \& Littlewood, 2006; Zea, Reisen, Poppen, Bianchi, \& Echeverry, 2005). Often, the very process of disclosure itself leads to negative consequences such as isolation, abandonment, and abuse (Lovejoy, 1990). For many women, disclosing one's HIV status may lead to negative consequences that impact not only the patient, but also their children (Schmidt \& Goggin, 2002).

Negative consequences of HIV disclosure, including increased depressive and anxiety symptoms have been illustrated in the literature. Unsupportive social and family interactions have been associated with elevated depressive symptoms in populations of HIV-positive women (Schrimshaw, 2003). Moreover, the idea of voluntarily disclosing one's HIV status, creating a situation that may lead to negative circumstances, is a daunting one. Women often rely on their partners for environmental safety and security, and fear that disclosure may lead to abandonment and rejection (Holt et al., 1998; Metcalfe, Langstaff, Evans, Paterson, \& Reid, 1998). Disclosure of HIV status for women has additional consequences that affect not only their intimate relationships, but also the safety and security of their children.

HIV literature illustrates the impact of stigma on HIV disclosure. Shame and stigma associated with HIV often prevents patients from disclosing their serostatus to friends, family members, and sexual partners (Jenkins \& Coons, 1996 ; Moneyham et al., 1996a, 1996b). Disclosure of one's HIV status is a complex process, with the stigma of the disease impacting both the reluctance to disclose and the aftermath of the disclosure (Vanable et al., 2006). Although stigmatization associated with HIV has decreased since the early years of the epidemic (Herek, Capitanio, \& Widaman, 2002), HIV continues to be surrounded in fear and stigma. Herek and colleagues (Herek et al., 2002) conducted a nationwide survey exploring stigma related to HIV and reported that one in five participants reported "fearing" someone with AIDS, while one in six admitted to having feelings of "disgust" about persons with AIDS. Therefore, the stigma of HIV has blanketed the disclosure process in fear and discrimination. Disclosure removes the veil of secrecy. The idea of taking medication in secrecy often leads to decreased medication adherence, increased denial of the disease, and further perpetuates the stigma and shame that is associated with HIV. HIV-related stigma has been associated with an increase in HIV-related symptoms, depressive symptoms, and receipt of recent psychiatric care (Vanable et al., 2006).

Although some published studies in the HIV literature reveal negative outcomes of HIV disclosure (Lovejoy, 1990; Vanable et al., 2006) most of the literature regarding the impact of disclosure on depressive and anxiety symptoms is positive (Schmidt \& Goggin, 2002; Zea et al., 2005). Disclosure is essential to obtaining social support (Moneyham et al., 1996a). HIV-positive women that disclose their status to a partner or close friend demonstrated lower levels of emotional distress (D. K. Smith et al., 1996). 
Schmidt and Goggin (Schmidt \& Goggin, 2002) found that through positive disclosure experiences, some HIV-positive women gain an increased perception of social support, resulting in fewer reported depressive symptoms. However, these researchers also noted that HIV-positive single mothers reported less satisfaction from the disclosure process, lower levels of perceived social support, and higher levels of depressive symptoms. The emotional support that is provided as a side effect of the disclosure is often the deciding factor in multiple daily activities which directly affect the lives of those infected with HIV, such as adherence with complex medication regimens and frequent medical appointments.

In a study of HIV-positive Latino gay and bisexual men ( $N=301)$, participants were recruited from HIV clinics, hospitals, and community agencies in three metropolitan cities in the United States (Zea et al., 2005). Men that disclosed their HIV status had lower levels of depression and greater levels of self-esteem than those men that did not disclose their HIV status. Quality of social support was associated with disclosure patterns. Disclosure was highest among closest friends $(85 \%)$, followed by primary male partner (78\%), followed by parents ( $37 \%$ to mother, $23 \%$ to father). Results indicate that Latino men were selective with whom they disclosed their HIV status, but experienced positive benefits in psychological well-being in the process. Despite the vast differences in sample demographics, this study informs the current study as both Latino men and African American women are minority populations and the groups shared the stigmatizing diagnosis of HIV.

Stein and colleagues (Stein et al., 1998) examined the disclosure patterns of HIVpositive persons $(N=203)$ to their sexual partners. Participants were recruited from two outpatient urban hospital clinics, where persons HIV-positive persons sought healthcare. The sample consisted of Black Americans (46\%), Latino (23\%), and White Americans $(27 \%)$. The majority of the sample was male $(69 \%)$, which reflects the transmission patterns and epidemiological history of HIV in the United States. The sample reported varying risks for HIV transmission, with sexual transmission being the primary risk factor (59\%). Twenty percent of the sample identified themselves as bisexual or homosexual males and $39 \%$ of the sample identified as heterosexual. Injection drug use was reported in $41 \%$ of the sample. Of the 203 persons that were interviewed, 129 reported sexual activity within the past 6 months. The majority $(60 \%)$ of the sample had disclosed their HIV status to all of their sexual partners. In the persons that had not disclosed their HIV status to their sex partner, half of them reported only one sex partner in their lifetime. Failure to consistently use condoms during sexual activity was reported in the majority of the sample (57\%) of nondisclosures. Multiple regression analysis revealed that persons with high levels of spousal support were 2.8 times more likely to disclose their HIV status to their partners than persons without high levels of support. Disclosure was 3.1 times more likely to occur in the white and Latino participants than the black participants.

HIV literature reveals that disclosure of HIV status may also have an impact on immune status. A study of $373 \mathrm{HIV}$-positive patients that were being treated in an outpatient psychiatric setting participated in a study that investigated the relationship 
between disclosure of HIV stats and disclosure of sexual orientation and their immune function. Patients that had disclosed their HIV status showed increased CD4 counts over time. Patients that had always been open about their sexual orientation also showed immunologic benefit of disclosure (Boggs, 2007).

Although disclosure of HIV status is a major aspect of living with HIV, research studies within the HIV literature regarding the disclosure process and ramifications of HIV disclosure vary widely. Dissimilar findings across studies were most likely due to differences in ways that disclosure was assessed (time from diagnosis, timing of disclosure, and hierarchy of order of persons disclosed to). Literature illustrates that stigma continues to retard the disclosure process. Moreover, failure to disclose HIV status can have devastating effects on public health. The most effective means of HIV prevention among sexually active persons is consistent condom use (Branson et al., 2006). Niccolai, Dorst, Myers, and Kissinger (Niccolai, Dorst, Myers, \& Kissinger, 1999) reported that lack of condom use among HIV-positive persons was prevalent in those that did not disclose their HIV status to their partner. Disclosure patterns are vital pieces of information with potential psychological ramifications, and should therefore be tracked and studied in the future.

Sleep Quality and Mood Disorders

Evidence continues to accumulate supporting the association between sleep disturbances and poor sleep quality with mood disorders. Research studies have shown that $10 \%$ to $40 \%$ of adults have intermittent insomnia, while $15 \%$ of all adults report long-term sleep difficulties (Ringdahl, Pereira, \& Delzell, 2004). Sleep quality has also been described as sleep efficiency, which is the ratio of total sleep time to time in bed or the proportion of sleep during the time period generally filled by sleep (Buysse, Reynolds, Monk, Berman, \& Kupfer, 1989).

Consequences of poor sleep quality can be complex. Although traditionally given less attention in prior research studies, estimation of sleep quality has become an important aspect of comprehensive medical assessments. Research supports that fact that people tend to overestimate their duration of sleep with subjective sleep assessments, therefore studies with both subjective and objective sleep measurements are needed to garner more precise data (Kripke et al., 2004). Poor sleep quality is a common complaint among patients with alcohol use disorders (Dahl et al., 2003) and persons with mood disorders (Kripke et al., 2004; Lee et al., 2001).

In a study of ethnicity and sleep, illumination, and mood disorders, Kripke and colleagues (Kripke et al., 2004) measured sleep with both objective and subjective means. The authors examined ethnic differences in sleep and depression in a sample $(\mathrm{N}=$ 459) of European American (72.2\%) and Hispanic (13.7\%), African American (9.4\%), Asian-pacific American (4.0\%) and Native American (0.7\%) postmenopausal women. In this population of women, Hispanic and African American slept less than less than European American women. 
Lee and Miramontes (Lee et al., 2001) examined the relationship between sleep, depression, and fatigue in a group of HIV-positive women $(N=100)$. Subjective measurements of depressive symptoms and fatigue were captured by self report questionnaires. Objective measurements of total sleep time, numbers of awakenings from sleep, daytime activity, and sleep efficiency were captured using motion sensor bracelets (wrist actigraphy). Consecutive activity was recorded for a continuous 48 hour time period. Lower depressive symptoms were noted in women with higher sleep efficiency.

Sleep disturbances are of particular concern in the HIV population. Adverse effects of poor sleep quality on immunity has been reported in healthy individuals and individuals who are HIV-positive (Cruess et al., 2005). Researchers have found high levels of sleep disturbances among the HIV-positive population (Lee et al., 2001; Mock et al., 2002; Rubinstein \& Selwyn, 1998). Insomnia is often one of the first symptoms reported in persons newly diagnosed with HIV (Mock et al., 2002). Prevalence rates of poor sleep quality vary across studies. Rubinstein and Selwyn (Rubinstein \& Selwyn, 1998 ) reported that $73 \%$ of their sample of HIV-positive persons reported sleep disturbances. Phillips and colleagues (Phillips et al., 2005) reported a 100\% prevalence rate of sleep disturbances as measured by the PSQI in their sample $(N=107)$ of HIVpositive men $(n=70)$ and HIV-positive women $(n=35)$. Mean PSQI score levels in this sample were $12.3 \pm 3.8$ were well above the cut-off score of 5 for poor sleep quality on the PSQI. African Americans were well represented in this study $(n=71,82.6 \%)$, and other demographic variables such as income and educational level were similar to those in the current study.

Similar results were noted in Mock and colleagues' 2002 (Mock et al., 2002) study which included a sample of exclusively African American HIV-positive women ( $N$ $=174$ ). Demographic characteristics of the sample were similar to the current study, especially marital status (52.3\% of the sample was single) and income $(54.9 \% \mathrm{oft}$ the sample had annual household income levels less than 10,000. This sample of African American women was at varying disease stages, and results were differentiated based on stage and severity of illness. Depressive symptoms were measured with the CES-D I (which eliminates somatic complaints) and sleep dysfunction was measured with the PSQI. Results illustrated a high rate of fatigue (45.8\%), depressive symptoms indicative of clinical depression (59\%), and sleep dysfunction (59\%). The authors noted that sleep disturbances often present before the diagnosis of HIV and become worse as severity of illness develops.

\section{Sexual Assault and Mood Disorders}

History of sexual assault has been associated with depression and anxiety disorders (Barbe et al., 2004; Barker-Collo \& Read, 2003; M. Conway et al., 2004; Lee et al., 2001; Mock et al., 2002; Peleikis, Mykletun, \& Dahl, 2004; Rubinstein \& Selwyn, 1998; Walrath et al., 2003). The long term effect that sexual abuse or assault has on an individual varies. Dickinson and colleagues (Dickinson, deGruy, Dickinson, \& Candib, 1998) describe a spectrum of resulting mood disorders, ranging from mild to complex symptoms. Dickinson enrolled 99 women in a study to evaluate the existence of complex 
post traumatic stress syndrome in women that report a history of sexual abuse. Results illustrated that varying degrees of symptoms were found in this group of women. The most severely abused subjects were characterized by symptom patterns similar to those of a complex post-traumatic stress syndrome. The type and duration of abuse often has a lot to do with long term consequences.

The prevalence of childhood sexual abuse has been documented to be between $15-30 \%$ in adult women and between $25-77 \%$ in adult women with diagnoses of mental illness (Lundqvist, Hansson, \& Svedin, 2004). In studies focused on the impact of sexual abuse or assault, associations with mood disorders have been reported (Barbe et al., 2004; Barker-Collo \& Read, 2003; M. Conway et al., 2004; Lee et al., 2001; Mock et al., 2002; Peleikis et al., 2004; Rubinstein \& Selwyn, 1998; Walrath et al., 2003).

Barbe and colleagues (Barbe et al., 2004) examined the relationship between history of sexual abuse and depression among 107 adolescent females. Subjects had a diagnosis of MDD by DSM-IIIR guidelines and were between the ages of 13 and 18 . Subjects were randomly assigned to one of three treatments: (1) cognitive behavioral therapy, (2) systemic behavioral family therapy, or (3) nondirective supportive therapy and then followed for three and a half years. Lifetime history of sexual abuse was captured as either present or absent. Results indicated that girls with a lifetime history of sexual abuse were more likely to have a psychiatric hospitalization or depressive relapse. Cognitive behavioral therapy was the most efficacious treatment in the absence of sexual abuse. Nondirective supportive therapy was most efficacious in the presence of sexual abuse. These results suggest the need to screen adolescent girls for history of sexual abuse as part of a psychosocial assessment.

Whereas most studies have focused on the emotional impact of sexual abuse, Girdler and colleagues (Girdler et al., 2004) examined a pathophysiologic impact of history of sexual abuse. These researchers examined the hypothalamic-pituitary-thyroid axis hormone concentrations in a group of women $(\mathrm{N}=50)$ during different phases of their ovulatory cycles. Increased total T3 concentrations were noted in women with histories of major depression; these increased concentrations also predicted their premenstrual symptoms. The results indicated that women with histories of sexual abuse had higher levels of conversion of T4 to T3 and increased binding of thyroid hormones in women with both premenstrual dysphoric disorder and history of sexual abuse. These results indicate that further pathophysiologic testing may be needed in women with mood disorders.

\section{SUMMARY OF THE REVIEW OF LITERATURE}

Despite advances in research, mood disorders are often undiagnosed and untreated in HIV-positive populations (Bing et al., 2001; M. Conway et al., 2004; Evans et al., 1996). Although many health care providers assume that depression is a natural, inevitable reaction to the diagnosis of HIV, patients who are HIV-positive are often under screened for mood disorders. Diagnosis of mood disorders within the HIV-positive population is complex, due to the overlapping somatic symptoms associated with 
HAART and signs and symptoms of specific HIV-related disorders (Norman et al., 1992; Perkins et al., 1994). The prevalence of mood disorders in the HIV population is higher than that of the general population. HIV has become a treatable chronic condition. Longitudinal studies are needed to identify varying levels of depressive and anxiety symptoms over time, examine underlying vulnerabilities to mood disorders in this population, as well as to examine optimal treatment therapies over time.

HIV-positive women are part of a unique population with additional risks for mood disorders. The majority of the responsibility of family caregiving falls on women, adding additional stressors (Hooyman \& Gonyea, 1999). HIV-positive women with children are more likely than men to suffer from chronic sorrow related to their illness, fear of death, poverty, and social isolation (Morrison et al., 2002). Although it is impossible to accurately predict with certainty those that will develop depressive or anxiety symptoms, the assessment of underlying vulnerabilities may aid the screening process. Many cross-sectional studies have investigated levels of depressive and anxiety symptoms at specific points of time, yet these studies do not account for the variety of symptoms that may occur at different points in time in the trajectory of HIV-infection. Most of the cross-sectional and longitudinal studies in the HIV literature support the positive association between HIV and anxiety and depressive symptoms. Although published studies examined predictors of depressive and anxiety symptoms among HIVpositive persons, demographic characteristics of the samples including race, gender, and sexual preference vary greatly, making generalizability to populations of African American HIV-positive women difficult.

HIV-positive women experience increased levels of depressive and anxiety symptoms. Therefore, the present study will address the relationships between underlying vulnerabilities; both inherited (family history of mood disorders, family history of alcohol and/or drug use) and acquired (educational level, income level, alcohol and/or drug use, HIV disclosure status, sleep quality, and history of sexual abuse and/or assault) to depressive and anxiety symptoms. Because these relationships have not been previously described using multiple risks as predictors in this population, findings will provide significant insights regarding the experiences of this understudied population of African American women who are HIV-positive. Specific aspects of the current study's methodology are presented in the next chapter. 


\section{CHAPTER 3. METHODOLOGY}

The incidence of HIV infection continues to increase, especially among the heterosexual population (Centers for Disease Control and Prevention, 2007c). HIV is a virus that is commonly spread through the exposure to infected blood and bodily fluids during sexual activity (Centers for Disease Control and Prevention, 2000, 2003). Studies demonstrate that there is a high prevalence of depression among HIV-positive persons (Bing et al., 2001; Ferrando et al., 1998; Rabkin, Wagner, \& Rabkin, 1999) as well as comorbid anxiety (Ciesla \& Roberts, 2001). Additionally, HIV-positive women are at an even greater risk for depression and anxiety (Bing et al., 2001; Ciesla \& Roberts, 2001; Moore et al., 1999). It is important that risk factors for depression and anxiety, both inherited and acquired, be identified. Early identification and treatment of depression and anxiety can have a direct impact on quality of life and treatment adherence in HIVpositive persons (Moore et al., 1999; Valente, 2003). The purposes of the study are fourfold: (1) to describe the level of depressive and anxiety symptoms in African American women who were HIV-positive; (2) to describe the underlying vulnerabilities, both inherited and acquired, for depressive and anxiety symptoms in African American women who were HIV-positive; (3) to identify which vulnerabilities serve as predictors of depressive and anxiety symptoms among African American HIV-positive women; and (4) to describe the differences in levels of depressive and anxiety symptoms among African American HIV-positive pregnant and African American women. This chapter explains the processes utilized to answer the research questions of this study. This chapter explicates the research design, setting and sample, recruitment, inclusion and exclusion criteria, sample size, instruments, procedures, consideration of human subjects, and data analysis.

\section{RESEARCH DESIGN}

A non-experimental, descriptive, correlation design was employed to obtain information on potential predictors of depressive and anxiety symptoms among African American HIV-positive women. This descriptive correlation design was used to accomplish the following objectives: 1) to describe the levels of anxiety and depressive symptoms among African American women who were HIV-positive, 2) to identify the inherited and acquired vulnerabilities that predict depressive and anxiety symptoms among African American women who were HIV-positive, 3) to quantify the inherited and acquired vulnerabilities that predict depressive and anxiety symptoms among African American women who were HIV-positive, 4) to determine the predictors of anxiety and depression symptoms among African American women who were HIV-positive, 5) to determine the differences in levels of depressive and anxiety symptoms among African American HIV-positive pregnant and African American HIV-positive non-pregnant women, 6) to determine the relationship between predictors of depressive and anxiety symptoms among African American HIV-positive pregnant and African American HIVpositive non-pregnant women, and 7) to determine the differences in levels of depressive and anxiety symptoms among African American HIV-positive pregnant and African American HIV-positive non-pregnant women. 
The study was cross-sectional as the variables were measured at a single point in time. All instruments that measured depressive and anxiety symptoms have established reliability and validity. According to Polit and Hungler (Polit \& Hungler, 1995), descriptive research is used to observe, describe, and document specific phenomena rather than explaining the phenomena. Correlation research examines relationships between variables (Polit \& Hungler, 1995).

In a review of pertinent literature, the incidence of depression among HIVpositive persons has been well documented, however, information regarding the triggers, or predictors of depression have not been fully explored. There was also a lack of information regarding anxiety among HIV-positive persons, although anxiety is often accompanied by a concomitant diagnosis of depression (Hirschfeld, 2001; J. M. Murphy et al., 2004). Therefore, the underlying predictors of both depressive and anxiety symptoms were addressed in this study.

\section{SETTING AND SAMPLE}

The setting was an outpatient university obstetrical and gynecological clinic and an outpatient adult infectious disease clinic that specializes in HIV. Both ambulatory clinics were located in the mid-south. Outpatient infectious disease clinics, the local county health department, and other obstetrical and gynecological clinics in the midsouth area often refer their HIV-positive, female clients to the outpatient university clinic for obstetrical and gynecological health care. Likewise, primary care providers, the local county health department, and other obstetrical and gynecological clinics in the midsouth area also refer their HIV-positive, female clients to the outpatient adult infectious disease clinic.

In the obstetrical clinic, a designated physician and nurse practitioner who had extensive infectious disease and HIV treatment and management experience, provided health care for this population of HIV-positive women. Although the clinic was open each weekday, HIV-positive women were seen on two designated days each week. Transportation and childcare were available to all HIV-positive women attending the study obstetrics and gynecology clinic through community support agencies.

The adult infectious disease clinic employed seven physicians and two nurse practitioners who had extensive infectious disease and HIV treatment and management experience and who provided health care for this population of HIV-positive patients. The clinic was open each weekday; HIV-positive men and women were seen Tuesday through Friday. Mondays were reserved for new HIV-positive patients. Transportation and childcare were available to all HIV-positive women attending the study clinics through community support agencies.

The study employed a non-probability convenience sample drawn from African American women, who were HIV positive, and were patients either in the outpatient obstetrics and gynecology or the university outpatient infectious disease clinic in an urban southeastern city. All women who met the inclusion criteria were potential 
participants. The target population was African American HIV-positive women in the mid-south, which was reflective of the HIV-infected population in the area, as well as in the university's clinic populations. This population was chosen because African American women are underrepresented in published studies examining depressive and anxiety symptoms in the HIV-positive population.

The obstetrics and gynecology clinic saw approximately 5000 women per month. HIV-positive women represented approximately $3 \%$ of the clinic population. Approximately $60 \%$ of the HIV-positive pregnant women seen in the practice were newly diagnosed, while approximately $40 \%$ were women who knew of their HIV status and had become pregnant. HIV-positive non-pregnant women receiving gynecological consultation accounted for $10 \%$ of the HIV-positive women receiving care at the university obstetrics and gynecology clinic. The infectious disease clinic exclusively saw patients who were HIV-positive. The infectious disease clinic saw approximately 350 patients per month. Forty percent of the clinic population was women; $75 \%$ of the population was African American. All African American HIV-positive women who received medical care at the designated clinics between September 1, 2006 and February 28, 2007 were approached for study participation. Of the 90 who were approached, 80 gave consent and completed the study.

\section{RECRUITMENT}

The clinic nurse, nurse practitioner, social worker, or physician informed the women who met study inclusion criteria about the study during their routine obstetrical or infectious disease medical appointments. The researcher developed an implementation plan that she discussed with clinic staff, and ensured that standardized recruitment techniques were maintained throughout enrollment. The researcher periodically verified that all interviewers appropriately implemented the recruitment plan, which provided consistency to the data collection process. Training regarding potential questions and answers, as well as an interview script was provided to the interviewers by the researcher. All interviewers participated in role play activities that simulated the interview and data collection process to ensure that plan was implemented appropriately and consistently. Providers gave interested women's contact information to the researcher. Women who expressed interest were contacted by the researcher. These contacts with potential participants by the investigator were either via telephone or through a personal visit during their routine clinic appointment. African American women who were HIVpositive and who agreed to participate and met the study criteria completed the informed consent process, signed the informed consent and completed the designated paper and pencil instruments.

\section{Inclusion Criteria}

The sample population was African American women who were HIV-positive, and were current patients in the university outpatient obstetrics and gynecology or

infectious disease clinic. The sample was recruited from HIV-positive women who were 
being treated in the special care clinic of the university obstetric and gynecologic clinic or the university infectious disease clinic. Criteria for inclusion into this study were as follows: 1) female gender, 2) self-identified as African American and non-Hispanic, 3) HIV-positive as documented by HIV-ELISA and HIV Western Blot test results abstracted from participant health records, 4) aged 18 years or greater on last birthday, 5) possessing ability to read and write in the English language as verified by highest educational level completed being at least the ninth grade, and 6) receiving medical care at the university obstetrics and gynecology or infectious disease clinic.

\section{Exclusion Criteria}

Women were excluded if they had existing co-morbid diseases that contributed to the potential inability of participants to answer questionnaires. Therefore participants were excluded from participating in the study if they had the following diagnoses: 1) HIV dementia, active Cytomegalovirus, or Toxoplasmosis; 2) mental retardation; and 3) latest CD4 cell count $<200$. Presence of compromised immune status, as measured by CD4 cell count less than 200 was collected from the medical record, and was used to determine if the participant was at risk for developing AIDS dementia, CMV, or toxoplasmosis, which could impact mental abilities, and possibly interfere with accurate completion of instrumentation. Exclusion criteria were determined through medical record review.

\section{Sample Size}

The sample size necessary to adequately conduct this study was determined by a power analysis calculated on the basis of multiple regression analysis. A sample of 80 participants was interviewed. To ensure an adequate sample size, a minimum of 80 African American HIV-positive women were recruited for this study. Due to the volume of patients available in each clinic, a three-to-one ratio was established by the researcher in order to obtain an adequate sample size (three non-pregnant women to every pregnant woman). A power analysis was performed to determine the number of subjects needed to ensure an alpha of .05 using the statistical package Power Precision. The effect size was chosen according to Cohen's criteria (Cohen, 1987) for a moderate effect size $(r=.30)$. This analysis ensured adequate numbers of subjects needed to detect clinically significant results. Using an alpha set at .05 , the proposed study had power of 0.80 with eight independent variables included in the prediction model.

\section{INSTRUMENTS}

The investigator used multiple paper and pencil instruments to address the variables of interest contained in the research questions. All instruments were placed in packets and were numbered for tracking purposes. A copy of each instrument is provided in Appendices A1-A4. Instruments that were included in each packet were the following: a researcher-developed demographic data questionnaire, Pittsburgh Sleep Quality Index 
(PSQI), Center for Epidemiological Studies Depression Scale (CES-D), Edinburgh Postnatal Depression Scale (EPDS), and the Beck Anxiety Inventory (BAI). Since the demographic data questionnaire was developed by the researcher and had not been used in the clinical setting, the instrument was administered to five randomly selected African American women who were HIV-positive. These women were then excluded from participating in the final study, as they had prior exposure to the instrumentation. This pilot test of the researcher-developed demographic data questionnaire was performed in order to determine the effectiveness of instructions, clarity of questionnaire items, and time required to complete the questionnaire. The pilot test of the researcher-developed questionnaire allowed the researcher to revise the instrument based on results of the pilot test.

The study instruments were selected based on a review of literature and professional experience, and their purpose was to gather specific information needed to investigate the variables included in the research questions. The PSQI (Buysse et al., 1989), CES-D (Radloff, 1977), EDPS (Cox et al., 1987) and the BAI (A. T. Beck et al., 1988) have been used in African American, female, and HIV-positive populations, and were conceptually congruent with the study. A blank at the top of each questionnaire was used for a code number to track respondents. Coding allowed the researcher to quantify the numbers of participants, as well as maintain confidentiality of the participants' identities.

\section{Demographic Data Instrument}

The researcher-developed demographic instrument (Appendix A) is a self-report and self-administered questionnaire that was designed to collect information regarding participant characteristics, and included: age, marital status, employment status, socioeconomic status (evaluated by reported annual income), educational level, religion, HIV disclosure status, reproductive history, mental health history, sexual abuse and/or assault history, alcohol and/or drug use, length of time since HIV diagnosis, and partner information. Family characteristics collected in the demographic data instrument included family history of mood disorders and family history of alcohol and/or drug use. Thus, both inherited and acquired vulnerabilities were assessed with this scale. Each item constructed for this instrument was subjected to testing with Question Understanding AID or QUAID (Graesser, Cai, Louwerse, \& Daniel, 2006). QUAID is a software program that assists survey designers to improve the wording, syntax, and semantics of survey questions. The tool identifies potential problems that survey respondents might have with understanding the meaning of survey items. Thus the software allows the survey item developer to review problems and improve the clarity of items and enhance reliability and validity of answers. The QUAID program picks up problems such as unfamiliar technical terms, vague or imprecise relative terms, vague or ambiguous noun phrases, complex syntax, and working memory overload. The demographic data instrument was pre-tested with five African American women who were HIV-positive in

order to identify confusing questions and ensure clarification. The 40-item instrument had an estimated completion time of five minutes. 
Sleep Quality Instrument

The Pittsburgh Sleep Quality Index (PSQI) was used to evaluate multiple dimensions of sleep quality (See Appendix B). The PSQI (Buysse et al., 1989) is a 19item, self-report, retrospective instrument designed to collect information regarding sleep quality and sleep disturbances over a one month period. The 19-items are grouped into seven equally weighted component scores including: 1) Subjective Sleep Quality (one item); 2) Sleep Latency (two items); 3) Sleep Duration (one item); 4) Habitual Sleep Efficiency (three items); 5) Sleep Disturbances (nine items); 6) Use of Sleeping Medication (one item); and 7) Daytime Dysfunction (two items). The PSQI has been widely used in a variety of populations, including populations of persons with chronic pain (Currie, Wilson, \& Curran, 2002; M. T. Smith, Perlis, Smith, Giles, \& Carmody, 2000), renal disease/transplant (Carpenter \& Andrykowski, 1998), cancer (Carpenter \& Andrykowski, 1998), and HIV (Currie et al., 2002; Mock et al., 2002; Nokes \& Kendrew, 1996; Nokes \& Kendrew, 2001; Phillips et al., 2005). The PSQI has also been used in populations of African American HIV-positive women (Mock et al., 2002).

Items one through four are participant entry of usual bed time, usual awake time, total minutes of sleep, and total minutes of sleep latency. Items five through 18 are fourpoint Likert-type scale responses related to frequency of problems, with zero indicating not during the past month; one indicating less than once a week; two indicating once or twice a week; and three indicating three or more times per week. Item 19 is a four-point Likert-type scale rating of overall sleep quality. A score of zero is very good, one is fairly good, two is fairly bad, and three is very bad.

The cumulative score range of the PQSI is zero to 21. Scoring the PSQI takes an estimated five minutes as scores from each item are added for a summed Global Score. Higher Global Scores are indicative of poorer sleep quality. A cut-off score of five or greater is indicative of poor sleep quality.

The PSQI has demonstrated reliability and validity. High internal consistency was demonstrated by Buyesse (Buysse et al., 1989), with a Cronbach's alpha coefficient of 0.83 for the seven component scores. Criterion validity was established in order to differentiate "good sleepers" from "poor sleepers". When compared to more costly and time consuming methods of sleep quality measurement (structured clinical interviews and polysomnographic testing), the PSQI Global score of five or greater correctly identified $88.5 \%$ of patients and controls in the original report (Buysse et al., 1989). Cronbach's alpha coefficient of the PQSI in the current study was 0.80 . The estimated time of completion is five to ten minutes.

\section{Depressive Symptom Instruments}

Two instruments were used to screen for depressive symptoms in the sample. This allowed the researcher to compare the participants' scores on two different instruments that were designed to capture similar information. This process also added to the body of 
knowledge regarding screening instruments in the African American population. This is imperative, as much of the published literature focuses on white populations.

The Center for Epidemiological Studies-Depression Scale (CES-D) was used in this study to measure the levels of depressive symptoms in the participants (see Appendix C). The CES-D (Radloff, 1977) was developed by the Center for Epidemiological Studies to measure the level of depressive symptoms in the general population. The CES-D has the ability to identify persons that experience depressive symptoms, but would not necessarily meet criteria to be diagnosed as clinically depressed (Moore et al., 1999). The CES-D is a screening instrument, and its use is not intended for the diagnosis of depression. The scale has been used extensively in the general and chronically ill populations (Radloff, 1977), including HIV-infected populations (Currie et al., 2002; Hudson et al., 2004; Lyketsos et al., 1996; Moore et al., 1999; Phillips et al., 2005), specifically HIV-positive women (Hudson et al., 2004; Ickovics et al., 2001; Mock et al., 2002). The CES-D has been used in populations of HIV-positive African Americans (Low-Beer et al., 2000; Mock et al., 2002; Robbins, Phillips, Dudgeon, \& Hand, 2004).

The CES-D contains 20 items, and assesses sleep disturbances, energy levels, feelings of guilt and depressed mood, and appetite loss in the respondents. The CES-D asks the individual to recall the presence of these issues in the prior week. Each item has a range of zero to three, with rarely or none of the time equaling zero points, and most or all of the time equaling three points. Scoring for positive items is reversed. Adding the sum of answers completes scoring. Possible total scores range from zero to 60, with the higher scores indicating the higher levels of depressive symptomatology. The total score on the CES-D can be divided into four distinct categories, with zero to 15 indicating no depressive symptoms, 16-20 mild distress, 21-30 moderate distress, and 31-60 severe distress (Radloff, 1977).

The CES-D is an accurate instrument, with high internal consistency and testretest reliability and can be completed in five to 10 minutes. Items for the CES-D were selected from several instruments, including: Beck's Depression Inventory (A. T. Beck, Rial, \& Rickels, 1974) the Minnesota Multi-phasic Personality Inventory, Raskin's Depression Scale (Raskin, Schulterbrandt, Reatig, \& McKeon, 1969), and Zung's Selfrating Depression Scale (Zung, 1965). Therefore, the CES-D has both construct and content validity.

The CES-D has been found to be reliable and valid in the general population, as well as populations of women (Fogel \& Martin, 1992; Hasin, Stinson, Ogburn, \& Grant, 2007; Hudson et al., 2004; Low-Beer et al., 2000; Phillips et al., 2005; Sullivan et al., 1996), including pregnant women (Hannah et al., 1992); and populations of both chronic and terminal diseases, including HIV (Currie et al., 2002; Hudson et al., 2004; Lyketsos et al., 1996; Moore et al., 1999; Phillips et al., 2005). The CES-D has demonstrated reliability and validity in African American women (Hudson et al., 2004; Ickovics et al., 2001; R. Lester \& Petrie, 1998; Liebschutz et al., 2003; Mock et al., 2002). Therefore, the CES-D is an appropriate instrument to use in a population of African American women 
who are HIV-positive. Cronbach's alpha coefficient of the CES-D in the current study was 0.90 .

The Edinburgh Postnatal Depression Scale (EPDS) was used in this study to measure the levels of depressive symptoms in the participants (see Appendix D). The EPDS (Cox et al., 1987) was developed for screening postpartum women for depressive symptoms. The EPDS has also been used as a screening tool in pregnancy (Murray, Cooper, \& Stein, 1991), and terminal illness (Lloyd-Williams, Friedman, \& Rudd, 2000). The EPDS has been well validated in both populations of women in the post-partum period (Chabrol \& Teissedre, 2004; Cox et al., 1987; Dennis, Janssen, \& Singer, 2004; Evins et al., 2000; Hannah et al., 1992) and populations of women with older children (Cox, Chapman, Murray, \& Jones, 1996).

Like the CES-D, the EPDS is a screening instrument and was not designed as an instrument to diagnose depression. The EPDS is an accurate instrument, with high sensitivity and specificity and test-retest reliability, and can be completed in less than five minutes. The EPDS was specifically developed for assessing postpartum depression, as other depression symptom instruments, specifically the Beck Depression Inventory (BDI) and the CES-D, illustrated elevated depressive symptom scores in symptomatic pregnant women due to the inclusion of somatic symptom assessments in the BDI and CES-D (Holcomb, Stone, Lustman, Gavard, \& Mostello, 1996; J. K. Myers \& Weissman, 1980).

The EPDS (Cox et al., 1987) is a 10-item questionnaire that asks subjects to report the severity of symptoms experienced in the last seven days. Each item on the EPDS has a range of zero to three, with rarely or none of the time equaling zero points, and most or all of the time equaling three points. Scoring for positive items is reversed. The sum of the scores is used as a total score. A score of ten or higher is indicative of the need for further screening. Total scores of 10-13 are indicative of mild depressive symptoms; scores greater than 13 are indicative of moderate depressive symptoms (Cox et al., 1987).

Literature supports the use of the minimum score (threshold) of 10 as predictive of postnatal depression (Chabrol \& Teissedre, 2004; Hannah et al., 1992). EPDS scores at five days postpartum have been highly correlated (Spearman rank correlation, $r=0.60 ; p$ $<.0001$ ) with EPDS scores at six weeks postpartum (Hannah et al., 1992). EPDS scores obtained at postpartum day two or three have been highly correlated (Spearman rank correlation, $r=0.59 ; p<.0001$ ) with EPDS scores obtained four to six weeks postpartum (Chabrol \& Teissedre, 2004).

There is a minimum score of zero and a maximum score of 30. Questions 3, 5-10 are reverse scored, discouraging the subject from answering the same way for each question. Question 10 addresses suicidal thoughts, and must be carefully assessed. If a subject has a total score less than ten, but notes suicidal tendencies, a referral should be made immediately. Prompt referral for those participants with EPDS scores less than ten, but with positive indications of suicidal thoughts, was ensured through immediate assessment after questionnaire completion. Cronbach's alpha coefficient of the EPDS in 
the current study was 0.91 . The EPDS values were positively correlated with the CES-D values in the present study, thereby illustrating convergent validity of the instruments.

\section{Anxiety Symptom Instrument}

The Beck Anxiety Inventory (BAI) [available at Pearson Education, Inc. (A. T. Beck et al., 1988)] was used in this study to measure the levels of anxiety symptoms in the participants. The BAI is a 21 -item instrument that asks subjects to report the degree of symptoms in the past week. Each item is scored on a four-point scale from zero to three. The items are summed to obtain a total score from zero to 63. A total score of zero to 21 is indicative of little or no anxiety symptoms. A total score of 22-35 is indicative of moderate anxiety symptoms, and a sum of 36 or greater is cause for concern (A. T. Beck et al., 1988). Each item is descriptive of subjective, somatic, or panic-related symptoms of anxiety. The BAI was developed to discriminate anxiety from depression by measuring anxiety symptoms that infrequently occur with depression. The BAI is selfadministered, but can also be administered verbally by a trained administrator. Approximately five to 10 minutes are required for completion of the BAI, and the instrument has been recommended in populations that are 17 years or greater or fifth grade reading level (American Psychiatric Association Task Force, 2000).

Reliability and both convergent and discriminate validity of the BAI have been established. The BAI has high internal consistency and item-total correlations that range from .30 to .71 (median= .60) (American Psychiatric Association Task Force, 2000). One-week test-retest reliability is .75. Positive correlation between the BAI and clinical evaluations has been illustrated. The BAI has been used in populations of women (Bauer, Costa, \& Hesselbrock, 2001). Internal consistency reliability for the study sample was calculated using Cronbach's alpha coefficient, and was 0.93.

\section{PROCEDURE}

The researcher requested permission to conduct the study from the University of Tennessee Health Science Center Institutional Review Board. Upon obtaining approval (Appendix E), the researcher secured use of the clinics for research purposes through the clinic manager and medical directors. A formal letter documenting this agreement is provided (Appendices $F$ and $G$ ). The purpose of the study was explained to the women by the clinic social worker (SW) during clinic visits. The clinic social worker was a vital part of the research team and was included in the IRB application process. The principle investigator (PI) was available to study participants for additional questions or concerns.

Women who expressed the desire to participate in the research study and met inclusion criteria were enrolled in the study. After obtaining verbal and written consent (Appendix H) the completed informed consents were placed in an envelope marked INFORMED CONSENTS. The questionnaires were assembled into research packets that were marked with a code number prior to their distribution. The SW maintained a list of participants and their corresponding code numbers. This process allowed a method of 
tracking the participants that needed additional assessments based on their scores on the CES-D (Radloff, 1977), EPDS (Cox et al., 1987), and BAI (A. T. Beck et al., 1988). The tracking process ensured that those subjects needing further assessment or immediate referral (suicidal tendencies as noted on the EPDS) were addressed promptly.

Participants completed the packets in the privacy of the examination room. The SW distributed the questionnaires, and the PI was available for questions during the questionnaire completion process. Instructions for questionnaire completion were verbally delivered by the SW, following a written script prepared by the PI. The participants were given instructions to complete the research packets, which included the Demographic Data Questionnaire, PSQI (Buysse et al., 1989), CES-D (Radloff, 1977), EPDS (Cox et al., 1987), and BAI (A. T. Beck et al., 1988). Participants were asked to not write their names or any other identifier on any of the instruments, as they were marked with code numbers. Completed questionnaires were reviewed by the SW for missing data. The SW was trained to assist participants with unanswered questions in a manner that allowed the participant to independently answer questions following instruction. Any missing data were obtained and then the packets were placed in a separate envelope marked, QUESTIONNAIRES. This procedure discouraged the identification of the subjects by the PI. The researcher was aware that although physical harm was not a danger in this study, much care was taken to prevent psychological harm to the participants. The PI provided time after the completion of the questionnaires for the participants to express thoughts and feelings.

Instrumentation was scored within a four hour period. This limited time frame allowed those participants needing further evaluation to be identified and referred to a health care provider for additional assessment. The signed informed consents and completed questionnaires were kept in a secure locked file cabinet within the PI's locked office. The SW maintained a list of all research participants and their corresponding code numbers. This allowed the PI to alert the SW of participants needing further evaluation without identifying the participant. The signed informed consents and the completed research questionnaires were secured and stored per IRB requirements.

\section{CONSIDERATION OF HUMAN SUBJECTS}

The rights of human subjects as study participants require protection and consideration. All requirements of the Health Information Privacy and Protection Act (HIPPA) were met and followed throughout the research process. The subjects' protected health information (PHI) collected in the study from medical records was used for research purposes only. The ethical principles of (1) respect for people, (2) beneficence, and (3) justice (National Institutes of Health, 1979) were observed during the conduct of this study.

The ethical principle of respect for persons requires researchers to obtain informed consent from research participants, maintain confidentiality, and protect participants with impaired decision-making capacity (Hulley, 2001). The Belmont Report (National Institutes of Health, 1979) describes the respect for persons as the recognition 
that individuals are autonomous agents. Respect for participants was illustrated when the subjects completed the informed consent process, as pertinent issues regarding risks, benefits, and alternatives to participation were covered in the informed consent (Appendix H).

The participants were guaranteed additional privacy with the anonymity that was built into the participant identification system. Each research packet was numbered, with the informed consent and questionnaires assigned a numerical code. Participants were asked to not include their names, initials, or any other identifying marks on the research materials. Confidentiality was ensured as the participants were informed that the researcher would not be able to identify them based on questionnaire responses. Participants were informed that their responses would be kept confidential, stored in a secure area, and destroyed in compliance with University of Tennessee Health Science Center Institutional Review Board procedures.

The ethical principle of beneficence in research requires that participants be protected from harm. Participants were protected from harm. The research study was designed to maximize benefits and minimize risks to the participants. This study involved minimal risk to the subjects. Psychological risks involved with being rejected as a subject were minimized through the consistent use of clearly defined inclusion and exclusion criteria. The benefits of the study were identification of women that needed additional assessment through collaboration with the clinic SW. Processes were in place to ensure that women needing additional screening were quickly referred. Follow-up was ensured by the SW.

The ethical principle of justice was ensured as the participants were informed that study participation was completely voluntary and refusing to participate in the study would not affect their access to medical care. Fair treatment of study participants was guaranteed through consistent instruction for questionnaire completion, as defined by the prepared script. These procedures helped ensure that all participants were treated in the same fashion throughout the screening, informed consent, and data collection process. No participant received a monetary incentive for participation.

\section{DATA ANALYSIS}

The SW examined all completed questionnaires; missing data were gathered by the SW prior to the subject's departure. Data were transferred entered onto an Excel spreadsheet with assigned code numbers in order to protect participants' confidentiality and anonymity. All data analysis was completed using the JMPTM statistical software program. The specific statistical analyses used to answer the research questions are explained in detail in the paragraphs that follow.

Nominal measurement was used to assign numbers to responses on the demographic data form, classifying characteristics into categories. Descriptive statistics including: measures of central tendency (i.e., mean and median), frequency distributions, 
and variability (i.e., standard deviation) were used to summarize and describe the demographic data.

\section{Research Question 1}

a. What are the levels of depressive symptoms in pregnant African-American women who are HIV-positive?

b. What are the levels of anxiety symptoms in pregnant African-American women who are HIV-positive?

c. What are the levels of depressive symptoms in non-pregnant African-American women who are HIV-positive?

d. What are the levels of anxiety symptoms in non-pregnant African-American women who are HIV-positive?

Research questions a through d were answered through the results of the CES-D, EPDS and BAI. Levels of depressive and anxiety symptoms in the sample were illustrated by their scores on the CES-D, EPDS, and BAI. These scores were continuous in nature, and results reported as descriptive statistics (i.e., mean, median, interquartile range, and standard deviation).

\section{Research Question 2}

a. What is the relationship of inherited vulnerabilities (family history of mood disorders, family history of alcohol and/or drug use) to depressive symptoms among pregnant African American women who are HIV positive?

b. What is the relationship of acquired vulnerabilities (educational level, income level, alcohol and/or drug use, HIV disclosure status, sleep quality, history of sexual abuse or assault) to depressive symptoms among pregnant African American women who are HIV positive?

c. What is the relationship of inherited vulnerabilities (family history of mood disorders, family history of alcohol and/or drug use) to depressive symptoms among non-pregnant African American women who are HIV positive?

d. What is the relationship of acquired vulnerabilities (educational level, income level, alcohol and/or drug use, HIV disclosure status, sleep quality, history of sexual abuse or assault) to depressive symptoms among non-pregnant African American women who are HIV positive?

Research questions $2 \mathrm{a}-2 \mathrm{~d}$ were answered using correlation statistical procedures. Independent variables for inherited vulnerabilities (family history of mood disorders, family history of alcohol and/or drug use) and acquired (educational level, income level, alcohol and/or drug use, HIV disclosure status, sleep quality, history of sexual abuse or assault) vulnerabilities were categorical in nature. Correlation analysis using Pearson correlation coefficient was used to describe the relationship between variables. The presence or absence of independent variables was addressed using dummy coding, with 
educational level, income level, alcohol and/or drug use, HIV disclosure status, sleep quality, history of sexual abuse or assault) presence being 1, and absence being 0 .

\section{Research Question 3}

a. What is the relationship of inherited vulnerabilities (family history of mood disorders, family history of alcohol and/or drug use) to anxiety symptoms among pregnant African American women who are HIV positive?

b. What is the relationship of acquired vulnerabilities (educational level, income level, alcohol and/or drug use, HIV disclosure status, sleep quality, history of sexual abuse or assault) to anxiety symptoms among pregnant African American women who are HIV positive?

c. What is the relationship of inherited vulnerabilities (family history of mood disorders, family history of alcohol and/or drug use) to anxiety symptoms among non-pregnant African American women who are HIV positive?

d. What is the relationship of acquired vulnerabilities (educational level, income level, alcohol and/or drug use, HIV disclosure status, sleep quality, history of sexual abuse or assault) to anxiety symptoms among non-pregnant African American women who are HIV positive?

Research questions 3a-3d were answered using correlation statistical procedures. Independent variables for inherited vulnerabilities (family history of mood disorders, family history of alcohol and/or drug use) and acquired (educational level, income level, alcohol and/or drug use, HIV disclosure status, sleep quality, history of sexual abuse or assault) vulnerabilities were categorical in nature. Correlation analyses were used to describe the relationship between variables. Correlation analysis using Pearson's correlation coefficient was used to describe the relationship between variables. Categorical variables were dummy coded as present (1) versus not present (0) to facilitate interpretation of study results.

\section{Research Question 4}

a. What is the influence of inherited vulnerabilities (family history of mood disorders, family history of alcohol and/or drug use) on the level of depressive symptoms reported by non-pregnant African-American women who are HIV positive?

b. What is the influence of acquired vulnerabilities (educational level, income level, alcohol and/or drug use, HIV disclosure status, sleep quality, history of sexual abuse or assault) on the level of depressive symptoms reported by non-pregnant African-American women who are HIV positive?

Research questions $4 \mathrm{a}$ and $4 \mathrm{~b}$ were answered using multiple regression analysis. The influence of the independent variables (inherited and acquired vulnerabilities) on the dependent variables (CES-D, EPDS scores) was established. Stepwise multiple regression analysis was performed in order to determine which independent variables 
best predicted the dependent variables. Dummy coding was used to identify the presence (1) or absence (0) of independent variables. In order to systematically test each variable, stepwise multiple regression with backward elimination was performed. Independent variables that illustrated statistically significant correlations were entered into the equation, and then one by one eliminated to test the influence of each independent variables (inherited and acquired vulnerabilities) on the dependent variables (CES-D, EPDS scores).

\section{Research Question 5}

a. What is the influence of inherited vulnerabilities (family history of mood disorders, family history of alcohol and/or drug use) on the level of anxiety symptoms reported by non-pregnant African-American women who are HIV positive?

b. What is the influence of acquired vulnerabilities (educational level, income level, alcohol and/or drug use, HIV disclosure status, sleep quality, history of sexual abuse or assault) on the level of anxiety symptoms reported by non-pregnant African-American women who are HIV positive?

Preliminary results revealed that the non-pregnant African American women who were HIV-positive had low levels of anxiety symptoms, as evidenced by BAI scores. Dummy coding was used to identify the presence (1) or absence (0) of independent variables. In order to systematically test each variable, stepwise multiple regression with backward elimination was performed. Independent variables that illustrated statistically significant correlations were entered into the equation, and then one by one eliminated to test the influence of each independent variables (inherited and acquired vulnerabilities) on the dependent variables (BAI scores).

\section{SUMMARY}

In this chapter, the design of the current study has been discussed. The limitations, setting, population, sample, instrumentation, and procedure were reviewed. The methods of protection of human subjects and data analysis were also identified. 


\section{CHAPTER 4. RESULTS}

The purposes of this study of HIV-positive women are fourfold: (1) to determine the levels of depressive and anxiety symptoms in pregnant and non-pregnant African American women who were HIV-positive; (2) to describe the association between underlying vulnerabilities, both inherited and acquired and depressive symptoms in pregnant and non-pregnant African American women who were HIV-positive; (3) to estimate the association between underlying vulnerabilities, both inherited and acquired, and anxiety symptoms in pregnant and non-pregnant African American women who were HIV-positive; and (4) to identify the predictors of depressive and anxiety symptoms among African American HIV-positive non-pregnant women.

Prior to conducting data analyses, subjects' responses to study questionnaires were entered into a Microsoft ${ }^{\mathrm{TM}}$ Excel spreadsheets then uploaded into the JMPTM statistical analysis software program. Descriptive statistics were estimated for each variable as were skewness and kurtosis scores to determine normality. Stem and leaf plots were also generated to assess normality. Many of the study variables were not normally distributed, resulting in the need to use nonparametric statistics for some analyses. For both independent and dependent variables that were normally distributed, mean scores with corresponding standard deviations were used to describe the data; for variables that were not normally distributed, the median $(M d n)$ and interquartile range $(I Q R)$ were reported. Finally, the results of each research question were addressed. Dummy coding was used to identify the presence (recoded as a score of 1) or absence (recoded as a score of 0 ) of specific categorical variables that were originally coded with more than two levels. This approach permitted the inclusion of these variables within regression analyses (Penhazur \& Schmelkin, 1991).

\section{DEMOGRAPHIC FINDINGS}

The mean age for the sample was $33 \pm 10.7$ years. The pregnant group was significantly younger than the non-pregnant group ( $24.4 \pm 4.58$ versus $36 \pm 10.57$ years; $t=-6.847, p<.0001)$ and had fewer children $(1.8 \pm 1.23$ vs. $2.49 \pm 1.82 ; t=-1.755, p=$ $.043)$. However, the groups did not differ significantly with regard to their education $\left(X^{2}\right.$ $=.207, d f=1, p=.768)$ and income $\left(X^{2}=.192, d f=1, p=.194\right)$ categories. The majority of the women in the present study were poor and unmarried. Most participants reported their marital status as single ( $n=14,70 \%$ of the pregnant women vs. $n=32,53.3 \%$ of the non-pregnant women). A few of the non-pregnant group $(n=6,10 \%)$ were widowed. One woman reported AIDS as the cause of her spouse's death; another woman reported that her husband committed suicide after she was hospitalized and diagnosed with AIDS. This participant also reported that after the suicide, she learned that her husband knew about his HIV diagnosis for many years prior to her diagnosis.

Almost half (49\%) of the total sample of pregnant and non-pregnant women reported annual household incomes of less than $\$ 5000$, (63\% of the pregnant women; $44 \%$ of the non-pregnant women). Employment was varied, with $47.5 \%$ of the total 
sample being unemployed, and $45 \%$ of the total sample being employed part time. Over half of the total sample ( $n=47,58.75 \%)$ had educational levels greater than high school graduate. Table 4.1 provides a sociodemographic summary of attributes of African American women who were HIV positive.

\section{INHERITED AND ACQUIRED VULNERABILITIES}

The investigator assessed inherited and acquired vulnerabilities using items included on the researcher's Demographic Questionnaire. Inherited vulnerabilities assessed included family history of mood disorders and family history of alcohol and/or drug use. Acquired vulnerabilities measured were educational level, income level, alcohol and/or drug use, HIV disclosure status, sleep quality, and history of sexual abuse or assault. Table 4.2 provides a summary of measures of inherited and acquired vulnerabilities for the total sample as well as for pregnant and non-pregnant women. In general, less than half of the total sample $(N=80)$ reported a positive family history of mood disorders (35\%) and family history of alcohol and/or drug use $(32.5 \%)$. One third of the participants $(n=25 ; 31 \%)$ reported alcohol and/or drug use, with the majority of those women being in the non-pregnant group. The majority of the total sample $(N=80)$ had educational levels of high school graduate or higher $(n=71 ; 88.75 \%)$. Low income levels (less than $\$ 5000$ /year) were seen in both groups of women, affecting over half $(63 \%)$ of the pregnant women and slightly less than half $(44 \%)$ of the non-pregnant women. The majority of the sample had disclosed their HIV status to at least one person ( $\mathrm{n}=74 ; 92.5 \%$ ), with sexual partners being the most commonly identified person. Poor sleep quality was prevalent in both groups of women, with $65 \%$ of the pregnant women and $62 \%$ of the non-pregnant women having PSQI scores indicative of poor sleep.

History of sexual assault was illustrated in both groups, with $35 \%$ of the pregnant and $44 \%$ of the non-pregnant group reporting a positive history of sexual assault. Current alcohol and/or drug use was higher in the non-pregnant group (40\%) than the pregnant group (5\%). Over one third of the total sample reported a history of mood disorder $(38 \%)$, with similar results noted in the two groups (30\% of pregnant women vs. $41 \%$ of non-pregnant women).

\section{AIM ONE}

Examine the levels of depressive and anxiety symptoms in African American women (pregnant and non-pregnant) who are HIV-positive in a southeastern urban university obstetrics and gynecology and infectious disease practice.

\section{All Women: Total Scale Scores for Outcome Variables}

Outcome variables were assessed based upon total scores from the CES-D, EPDS, and BAI for the entire sample $(N=80)$ of both pregnant $(n=20)$ and non-pregnant $(n=60)$ women. Average scores of depressive and anxiety symptoms are summarized in Table 4.3. The total score of the CES-D, a measure of depressive symptoms, is the sum of all 
Table 4.1 Sociodemographic Attributes of African American Women Who Were HIVpositive.

\begin{tabular}{|c|c|c|c|c|c|c|}
\hline & \multicolumn{2}{|c|}{$\begin{array}{c}\text { Total } \\
(N=80)\end{array}$} & \multicolumn{2}{|c|}{$\begin{array}{c}\text { Pregnant } \\
(n=20)\end{array}$} & \multicolumn{2}{|c|}{$\begin{array}{c}\text { Non-Pregnant } \\
(n=60)\end{array}$} \\
\hline & $n$ & $(\%)$ & $n$ & $(\%)$ & $n$ & $(\%)$ \\
\hline \multicolumn{7}{|l|}{ Income (annual) } \\
\hline$<5000$ & 38 & $(48.72 \%)$ & 12 & $(63.16 \%)$ & 26 & $(44.07 \%)$ \\
\hline $5000-10,000$ & 17 & $(21.8 \%)$ & 1 & $(5.26 \%)$ & 16 & $(27.12 \%)$ \\
\hline $10,000-15,000$ & 11 & $(14.1 \%)$ & 4 & $(21.05 \%)$ & 7 & $(11.86 \%)$ \\
\hline $15,000-25,000$ & 8 & $(10.26 \%)$ & 1 & $(5.26 \%)$ & 7 & $(11.86 \%)$ \\
\hline 25,000 or higher & 4 & $(5.13 \%)$ & 1 & $(5.26 \%)$ & 3 & $(5.09 \%)$ \\
\hline \multicolumn{7}{|l|}{$\begin{array}{l}\text { Educational } \\
\text { (highest completed) }\end{array}$} \\
\hline $9^{\text {th }}-11^{\text {th }}$ grade & 9 & $(11.25 \%)$ & 2 & $(10 \%)$ & 7 & $(11.86 \%)$ \\
\hline HS/GED & 24 & $(30 \%)$ & 6 & $(30 \%)$ & 18 & $(30 \%)$ \\
\hline Some College & 28 & $(35 \%)$ & 8 & $(40 \%)$ & 20 & $(33.33 \%)$ \\
\hline Bachelor's Degree & 18 & $(22.5 \%)$ & 4 & $(20 \%)$ & 14 & $(23.33 \%)$ \\
\hline Master's Degree & 1 & $(1.25 \%)$ & 0 & & 1 & $(1.67 \%)$ \\
\hline \multicolumn{7}{|l|}{ Employment Status } \\
\hline Unemployed & 21 & $(26.25 \%)$ & 6 & $(30 \%)$ & 15 & $(25 \%)$ \\
\hline Employed FT & 5 & $(6.25 \%)$ & 2 & $(10 \%)$ & 3 & $(5 \%)$ \\
\hline Employed PT & 36 & $(45 \%)$ & 10 & $(50 \%)$ & 26 & $(43.33 \%)$ \\
\hline Unemployed But looking & 17 & $(21.25 \%)$ & 2 & $(10 \%)$ & 15 & $(25 \%)$ \\
\hline \multicolumn{7}{|l|}{ Marital Status } \\
\hline Single & 46 & $(57.5 \%)$ & 14 & $(70 \%)$ & 32 & $(53.33 \%)$ \\
\hline Married & 14 & $(17.5 \%)$ & 2 & $(10 \%)$ & 12 & $(20 \%)$ \\
\hline Divorced & 3 & $(3.75 \%)$ & 1 & $(5 \%)$ & 2 & $(3.33 \%)$ \\
\hline Widowed & 4 & $(7.5 \%)$ & 0 & & 6 & $(10 \%)$ \\
\hline Cohabitating & 11 & $13.75 \%)$ & 3 & $(15 \%)$ & 8 & $(13.33 \%)$ \\
\hline
\end{tabular}

Note: HS=High School; GED= General Education Equivalent; PT= Part Time; FT= Full Time. Between group differences were not significant. 
Table 4.2 Inherited and Acquired Vulnerabilities Reported by the Total Sample and Pregnant and Non-Pregnant HIV-positive African American Women.

\begin{tabular}{|c|c|c|c|c|}
\hline Vulnerability & $\begin{array}{c}\text { Total } \\
N=80 \\
n(\%)\end{array}$ & $\begin{array}{c}\text { Pregnant } \\
n=20 \\
n(\%) \\
\end{array}$ & $\begin{array}{c}\text { Non- } \\
\text { Pregnant } \\
n=60 \\
n(\%)\end{array}$ & $\begin{array}{c}\text { Significance } \\
\text { Between } \\
\text { Pregnant and } \\
\text { Non-Pregnant } \\
p \text {-value } \\
\end{array}$ \\
\hline \multicolumn{5}{|l|}{ Inherited Vulnerabilities } \\
\hline $\begin{array}{l}\text { Family history of mood } \\
\text { disorder }\end{array}$ & $28(35.0)$ & $6(30.0)$ & $22(37.0)$ & 0.59 \\
\hline $\begin{array}{l}\text { Family history of alcohol } \\
\text { and/or drug use }\end{array}$ & $26(32.5)$ & $4(20.0)$ & $22(37.0)$ & 0.16 \\
\hline Acquired Vulnerabilities & & & & \\
\hline $\begin{array}{l}\text { Current alcohol and/or } \\
\text { drug use }\end{array}$ & $25(31 \%)$ & $1(5 \%)$ & $24(40 \%)$ & 0.0011 \\
\hline $\begin{array}{l}\text { Educational level } \\
\text { (High School graduate or } \\
\text { higher) }\end{array}$ & $71(88.75 \%)$ & $18(90 \%)$ & $53(88.33 \%)$ & 0.88 \\
\hline $\begin{array}{l}\text { Income level } \\
\qquad(<\$ 5000.00 / \text { year })\end{array}$ & $38(49 \%)$ & $12(63 \%)$ & $26(44 \%)$ & 0.26 \\
\hline Disclosed HIV status & $74(92.5 \%)$ & $19(95 \%)$ & $55(92 \%)$ & 0.59 \\
\hline $\begin{array}{l}\text { Poor sleep quality } \\
\quad(\mathrm{PSQI}>5)\end{array}$ & $50(62.5 \%)$ & $13(65 \%)$ & $37(62 \%)$ & 0.40 \\
\hline $\begin{array}{l}\text { History of sexual abuse or } \\
\text { assault }\end{array}$ & $33(42 \%)$ & $7(35 \%)$ & $26(44 \%)$ & \\
\hline
\end{tabular}

Note. $*$ PSQI $=$ Pittsburgh Sleep Quality Instrument 
Table 4.3 Descriptive Statistics for Depressive and Anxiety Symptoms in African American HIV-positive Women.

\begin{tabular}{lcccc}
\hline Outcome variable & $N$ & $M \pm S D$ & $M d n$ & $I Q R$ \\
\hline CES-D & 80 & $18.35 \pm 13.19$ & 17 & 23.75 \\
EPDS & 80 & $9.04 \pm 7.3$ & 6.5 & 12.75 \\
BAI & 80 & $11.2 \pm 11.54$ & 7 & 16 \\
\hline
\end{tabular}

Note: CES-D=Center for Epidemiologic Studies Depression Scale; EPDS=Edinburgh Postnatal Depression Scale; BAI=Beck Anxiety Inventory 
item responses. Results ranged from 0 to 60 with a cut-off score of 16 reflecting clinically relevant depressive symptoms in the general population (Radloff, 1977). Total CES-D scores for the study sample ranged from 3 (fewest depressive symptoms) to 53 (most depressive symptoms). Less than half of the sample $(n=38,47.5 \%)$ had CES-D scores less than 16, which indicated the absence of significant depressive symptoms (Radloff, 1977). Twelve (15\%) women had CES-D scores of 16-20 indicating clinically mild depressive symptoms, 12 (15\%) women had scores of 21-30 indicating moderate depressive symptoms, and 18 (22.5\%) women had scores greater than 31 indicating severe distress (Radloff, 1977). A total of $42(52.5 \%)$ women in the sample $(N=80)$ had CES-D scores of 16 or greater requiring referral to their case manager for follow-up.

The total score of the EPDS is the sum of all item responses and can range from 0 to 30 with a cut-off score of 10 indicating a need for further screening for depression (Cox et al., 1987). Total scores for depressive symptoms with the EPDS for the study sample ranged from 0 (fewest depressive symptoms) to 26 (most depressive symptoms). The majority of the sample $(n=44,55 \%)$ had EPDS scores of less than nine, which indicated the absence of significant depressive symptoms (Cox et al., 1987). Thirteen women $(16.25 \%)$ had EPDS scores between 10 and 13, indicating clinically mild depressive symptoms; and 23 (28.75\%) had EPDS scores higher than 13, indicating moderate to severe depressive symptoms (Cox et al., 1987). All participants with elevated scores were reported to their case manager for follow-up. Table 4.4 illustrates levels of specific categories of depressive symptoms.

Anxiety scores for the sample, which reflected the sum of all items, were lower than expected. Most of the sample had BAI scores below the level suggestive of the need for additional screening (Table 4.2). BAI scores for the total sample of both pregnant and non-pregnant women ranged from zero to 45 , with the majority $(n=69,86.25 \%)$ of the women exhibiting BAI scores of less than 21, illustrating little or no anxiety (A. T. Beck et al., 1988). BAI scores in the 22-35 range are indicative of moderate levels of anxiety (A. T. Beck et al., 1988). A small proportion of the total sample $(n=6 ; 7.5 \%)$ had BAI scores in the moderate range (A. T. Beck et al., 1988). BAI scores that were 36 and greater were indicative a high levels of anxiety and cause for concern (A. T. Beck et al., 1988). Five (6.25\%) women in the total sample exhibited high levels of anxiety based on their BAI scores. Table 4.5 illustrates the specific levels of anxiety symptoms in the total group of African American women who were HIV-positive.

Pregnant Women: Total Scale Scores for Outcome Variables

Levels of depressive symptoms in pregnant African-American women who were HIV-positive were also examined. Data were assessed for symmetry, or skewness, using Pearson's skewness coefficient. Perfectly symmetrical distributions have equal means and medians, with a Pearson skewness coefficient of 0 (Penhazur \& Schmelkin, 1991). Pearson skewness coefficient results that fall between -1 and +1 are acceptable, indicating data that are normally distributed. Values above or below -1 and +1 are substantially skewed distribution (Penhazur \& Schmelkin, 1991). Depressive symptoms, 
Table 4.4 Descriptive Statistics for Specific Levels of Depressive Symptoms in Total Sample of African American HIV-positive Women $(\mathrm{N}=80)$.

\begin{tabular}{lccccc}
\hline CES-D Scores & $N$ & $\%$ & EPDS Scores & $N$ & $\%$ \\
\hline None (0-15) & 38 & $47.5 \%$ & None $(0-9)$ & 44 & $55 \%$ \\
Mild (16-20) & 12 & $15 \%$ & Mild (10-13) & 13 & $16.25 \%$ \\
Moderate (21-30) & 12 & $15 \%$ & Moderate to Severe $(\geq 14)$ & 23 & $28.75 \%$ \\
Severe $(\geq 31)$ & 18 & $22.5 \%$ & & & \\
\hline
\end{tabular}

Note: $\mathrm{CES}-\mathrm{D}=$ Center for Epidemiologic Studies Depression Scale; EPDS=Edinburgh Postnatal Depression Scale 
Table 4.5 Descriptive Statistics for Specific Levels of Anxiety Symptoms in Total Sample of African American HIV-positive Women $(\mathrm{N}=80)$.

\begin{tabular}{lcc}
\hline BAI Scores & $n$ & $\%$ \\
\hline None to mild (0-21) & 69 & 86.25 \\
Moderate (22-35) & 6 & 7.5 \\
Severe $(\geq 36)$ & 5 & $6.25 \%$ \\
\hline
\end{tabular}

Note: BAI= Beck Anxiety Inventory 
as measured using the CES-D, were positively skewed (Pearson's skewness coefficient 0.78). Data were treated for the purposes of statistically analysis as normally distributed. As seen with the CES-D, depressive symptom scores measured using the EPDS (mean 8.7; median 5.5) were dissimilar, although the Pearson's skewness coefficient was 0.34, which is less than +1 , indicating data that are normally distributed. Results indicated that as a group, almost half of the sample (45\%) of pregnant African-American women who were HIV-positive had CES-D scores indicative of the need for referral and additional assessment of depressive symptoms. Consequently, over half of the pregnant women in the sample (55\%) reported depressive symptoms below the recommended cut-off for the CES-D (total score < 16) (Radloff, 1977). The median score of the CES-D in this group of pregnant women was 9 (interquartile range of 27.5). Results were similar for the EPDS. A score of 0-9 is indicative of the absence of clinically relevant depressive symptoms (Cox et al., 1987). EPDS scores for this group of pregnant women were below the recommended cut-off needed for further evaluation (median 5.5; interquartile range 14). On average, for both measures pregnant women did not have clinically relevant levels of depressive symptoms. Table 4.6 illustrates levels of depressive symptoms in this sample of pregnant African American women who were HIV-positive.

Levels of anxiety symptoms in pregnant African-American women who were HIV-positive were positively skewed (Pearson's skewness coefficient 1.29), and greater than +1 , indicating data that were not normally distributed. Results indicated that pregnant African-American women in the sample who were HIV-positive did not report anxiety symptoms that needed further evaluation. The majority of the sample of pregnant women exhibited anxiety symptoms below the recommended cut-off of 21 for the BAI (A. T. Beck et al., 1988). The median for BAI scores in this group was 5.5 and the interquartile range 19.5. Pregnant women in this sample did not have clinically relevant anxiety symptom levels. Table 4.7 illustrates levels of anxiety symptoms in this group of pregnant African American women who were HIV-positive.

\section{All Non-Pregnant Women: Total Scale Scores for Outcome Variables}

Levels of depressive symptoms in non-pregnant African-American women who were HIV-positive were examined using descriptive statistics. Results indicated that the levels of depressive symptoms in non-pregnant African-American women who were HIV-positive were positively skewed for both the CES-D (Pearson's skewness coefficient (0.56) and EPDS (Pearson's skewness coefficient 0.6). These Pearson's skewness coefficient values reflected data that were approximately normally distributed.

Results for aim one indicated that the typical non-pregnant African-American women in the study sample who were HIV-positive had mild depressive symptoms when measured by the CES-D (mean 18.6; standard deviation 12.53) and the EPDS (mean 9.15; standard deviation 7.29). CES-D scores for the typical non-pregnant women in this sample ranged from 3 to 46. EPDS scores for the typical non-pregnant women in this sample ranged from 0 to 26. In this group of non-pregnant African American HIVpositive women, CES-D and EPDS scores suggest that depressive symptoms were typically mild. Current treatment with antidepressants was not an exclusion criterion for 
Table 4.6 Descriptive Statistics for Specific Levels of Depressive Symptoms in Sample of Pregnant African American HIV-positive Women $(n=20)$.

\begin{tabular}{lccccc}
\hline CES-D Scores & $n$ & $\%$ & EPDS Scores & $N$ & $\%$ \\
\hline None $(0-15)$ & 11 & $55 \%$ & None $(0-9)$ & 11 & $55 \%$ \\
Mild $(16-20)$ & 1 & $5 \%$ & Mild $(10-13)$ & 3 & $15 \%$ \\
Moderate $(21-30)$ & 1 & $5 \%$ & Moderate to Severe $(\geq 14)$ & 6 & $30 \%$ \\
Severe $(\geq 31)$ & 7 & $35 \%$ & & & \\
\hline
\end{tabular}

Note: CES-D=Center for Epidemiologic Studies Depression Scale; EPDS=Edinburgh Postnatal Depression Scale 
Table 4.7 Descriptive Statistics for Specific Levels of Anxiety Symptoms in Sample of Pregnant African American HIV-positive Women $(n=20)$

\begin{tabular}{lcc}
\hline BAI Scores & $n$ & $\%$ \\
\hline None to mild (0-21) & 17 & 85 \\
Moderate $(22-35)$ & 3 & 15 \\
Severe $(\geq 36)$ & 0 & 0 \\
\hline
\end{tabular}

Note: BAI= Beck Anxiety Inventory 
the current study. Nine $(15 \%)$ of the non-pregnant women $(n=60)$ were on antidepressants at the time of their participation in the study, although eight of these women had elevated depressive symptom scores. Table 4.8 illustrates levels of depressive symptoms in this sample of non-pregnant African American women who were HIVpositive.

Levels of anxiety symptoms in the typical non-pregnant African-American women who were HIV-positive were examined. Results were positively skewed (Pearson's skewness coefficient 1.29), and greater than +1 , indicating data that were not normally distributed. Results indicated that most of the non-pregnant African-American women in the study who were HIV-positive had little or no anxiety (median 7, interquartile range 16). Anxiety scores in this group of non-pregnant women ranged from zero to 45 . Table 4.9 illustrates levels of anxiety symptoms in this group of non-pregnant African American women who were HIV-positive.

The Wilcoxon Signed Rank Sums test was performed to assess the significance of between group differences (pregnant versus non-pregnant) for each outcome. Depression scores as measured by the CES-D were similar $(p=.486)$ in both groups as was EPDS scores $(p=.743)$. Anxiety scores were also similar for the groups $(p=.558)$. Table 4.10 depicts a summary of the results of depressive and anxiety symptom scores in pregnant and non-pregnant women. As a group neither pregnant nor non pregnant women in the study sample had clinically relevant levels of depressive or anxiety symptoms.

\begin{abstract}
AIM TWO
Examine the associations among inherited (family history of mood disorders, family history of alcohol and/or drug use) and acquired (educational level, income level, alcohol and/or drug use, HIV disclosure status, sleep quality, history of sexual abuse or assault) vulnerabilities and depressive symptoms in pregnant and non-pregnant African American women who were HIV-positive.

Correlation statistics were used to analyze the relationship of inherited vulnerabilities (e.g. family history of mood disorders, family history of alcohol and/or drug use) to depressive symptoms among pregnant African American women who were HIV positive. Table 4.11 summarizes the correlation analyses in this group of pregnant women, illustrating the statistical significance of the correlation between family history of mood disorders and depressive symptoms.

Family history of mood disorders was captured on the Demographic Questionnaire. Results were dummy coded, with no family history of mood disorder coded as 0 and positive family history of mood disorders coded as 1 . There was a moderate but significant association between family history of mood disorders and depressive symptoms on the CES-D $(r=0.58 ; p=0.0073)$. Results for the EPDS were similar $(r=0.59 ; p=.058)$, however the level of significance was slightly above the A priory alpha of $p \leq 0.05$. Thus, family history of mood disorders was associated with
\end{abstract}


Table 4.8 Descriptive Statistics for Specific Levels of Depressive Symptoms in Sample of Non-Pregnant African American HIV-positive Women $(n=60)$.

\begin{tabular}{lccccc}
\hline CES-D Scores & $n$ & $\%$ & EPDS Scores & $N$ & $\%$ \\
\hline None $(0-15)$ & 27 & $45 \%$ & None $(0-9)$ & 33 & $55 \%$ \\
Mild (16-20) & 11 & $18.3 \%$ & Mild (10-13) & 10 & $16.7 \%$ \\
Moderate (21-30) & 11 & $18.3 \%$ & Moderate to Severe $(\geq 14)$ & 17 & $28.3 \%$ \\
Severe $(\geq 31)$ & 11 & $18.3 \%$ & & & \\
\hline
\end{tabular}

Note: CES-D=Center for Epidemiologic Studies Depression Scale; EPDS=Edinburgh Postnatal Depression Scale 
Table 4.9 Descriptive Statistics for Specific Levels of Anxiety Symptoms in Sample of Non-Pregnant African American HIV-positive Women $(n=$ $60)$.

\begin{tabular}{lcc}
\hline BAI Scores & $n$ & $\%$ \\
\hline None to mild $(0-21)$ & 52 & 86.7 \\
Moderate $(22-35)$ & 3 & 5 \\
Severe $(\geq 36)$ & 5 & 8.3 \\
\hline
\end{tabular}

Note: BAI= Beck Anxiety Inventory 
Table 4.10 Depressive and Anxiety Symptom Scores in Pregnant $(n=20)$ and NonPregnant $(n=60)$ African American HIV-positive Women.

\begin{tabular}{ccc}
\hline & Pregnant $(n=20)$ & Non-pregnant $(n=60)$ \\
Outcome & $M d n(I Q R)$ & $M d n(I Q R)$ \\
\hline CES-D & $9(27.5)$ & $17(21)$ \\
EPDS & $5.5(14)$ & $8(11.75)$ \\
BAI & $5.5(19)$ & $7(16)$ \\
\hline
\end{tabular}

Note: Median (Mdn) and interquartile ranges (IQR) were reported for data that were not normally distributed. CES-D=Center for Epidemiologic Studies Depression Scale; EPDS=Edinburgh Postnatal Depression Scale; BAI=Beck Anxiety Inventory. Between group differences were non-significant. The study a priori alpha was $p \leq .05$. 
Table 4.11 Pearson Correlation ( $r$ ) Analysis between Inherited Vulnerabilities and Depressive Symptoms for Pregnant African American Women Who Were HIV-positive $(n=20)$.

\begin{tabular}{lcc}
\hline & $\begin{array}{c}\text { Depressive Symptoms } \\
\text { (CES-D Scores) } \\
\text { Inherited Vulnerability }\end{array}$ & $\begin{array}{c}\text { Depressive Symptoms } \\
\text { (EPDS Scores) } \\
r(p \text { value })\end{array}$ \\
\hline Family History of Mood Disorders & $.58(.0073)$ & $.59(.058)$ \\
Family History of Alcohol/Drug Use & $.30(.20)$ & $.19(.42)$ \\
\hline
\end{tabular}

Note: $\mathrm{CES}-\mathrm{D}=$ Center for Epidemiologic Studies Depression Scale; EPDS=Edinburgh Postnatal Depression Scale. A priori alpha for the study was $p \leq 0.05$. 
depressive symptoms in this group of pregnant African American women who were HIVpositive. Women with a positive family history of mood disorders had higher depression scores than women with a negative family history on both measures of depressive symptoms.

Family history of alcohol and/or drug use was captured on the Demographic Questionnaire. Results were dummy coded with no family history of alcohol and/or drug use coded as 0 and positive family history of alcohol and/or drug use coded as 1 . There was a non-significant association between family history of alcohol and/or drug use with depressive symptoms on both the CES-D $(r=0.30 ; p=0.20)$ and the EPDS $(r=0.19 ; p=$ $.42)$. Thus, family history of alcohol and/or drug use was not significantly associated with depressive symptoms as measured by the CES-D, or the EPDS in this group of pregnant African American women who were HIV-positive.

Correlation statistics were used to analyze the relationship of acquired vulnerabilities (educational level, income level, alcohol and/or drug use, HIV disclosure status, sleep quality, history of sexual abuse or assault) to depressive symptoms among pregnant African American women who were HIV positive. Table 4.12 provides the complete results of these correlation analyses

Two categories of educational level were used for analysis, HS/GED (coded as 0) and HS/GED or higher (coded as 1). Educational level was not significantly associated with depressive symptoms when measured with the CES-D $(r=-0.12 ; p=0.62)$ or EPDS $(r=-0.09 ; p=0.72)$. Thus, educational level was not significantly associated with depressive symptoms in this group of pregnant African American women who were HIV-positive.

Income level was captured on the Demographic Questionnaire as annual household income in a categorical fashion. Five categories of income were collapsed into two categories, which was a reflection of the sample income distribution. Income level was coded 0 if income was less than $\$ 5000 /$ year and 1 if participants income was $\$ 5000 /$ year or greater. Income level was not significantly correlated with depressive symptoms when measured be the CES-D $(r=-0.06 ; p=0.80)$ or the EPDS $(r=0.09 ; 8 ; p$ $=0.69$ ). Thus, socioeconomic level was not significantly associated with depressive symptoms in this group of pregnant African American women who were HIV-positive.

The association between substance use and depressive symptom scores on the CES-D were non-significant $(r=0.22 ; p=0.35)$. Likewise, the correlation between alcohol and/or drug use and depressive symptoms using the EPDS was also nonsignificant $(r=0.42, p=0.07)$. Thus, substance use was not significantly associated with depressive symptoms in this group of pregnant African American women who were HIV-positive.

Disclosure of HIV status was measured on the Demographic Questionnaire by the number of persons to whom the subject had disclosed her HIV status. Pregnant women 
Table 4.12 Pearson Correlation ( $r$ ) Analysis between Acquired Vulnerabilities and Depressive Symptoms for Pregnant African American Women Who Were HIV-positive $(n=20)$.

\begin{tabular}{lcc}
\hline Acquired Vulnerability & $\begin{array}{c}\text { CES-D Depressive } \\
\text { Symptoms Scores } \\
r(p \text { value })\end{array}$ & $\begin{array}{c}\text { EPDS Depressive } \\
\text { Symptoms Scores } \\
r(p \text { value })\end{array}$ \\
\hline Income & $-.01(.80)$ & $.010(.69)$ \\
Educational Level & $-.12(.62)$ & $-.09(.72)$ \\
Alcohol and/or Drug Use & $.22(.35)$ & $.42(.07)$ \\
HIV Disclosure Status & $.54(.01)^{*}$ & $.37(.11)$ \\
History of Sexual Assault & $.28(.23)$ & $.27(.24)$ \\
Sleep Quality & $.61(.0004)^{*}$ & $.67(.0001)^{*}$ \\
\hline
\end{tabular}

Note: CES-D=Center for Epidemiologic Studies Depression Scale; EPDS=Edinburgh Postnatal Depression Scale. *The study a priori alpha was $p \leq .05$. 
Prior to conducting zero-order correlation analysis, nominal level variables that reflected was implemented to signify no disclosure to others (coded 0 ) or disclosure to others (coded 1). HIV disclosure status was significantly correlated with depressive symptoms when measured by the CES-D $(r=0.54 ; p=0.01)$, but not with the EPDS $(r=$ $0.37 ; p=0.11$ ) in this pregnant subgroup. Therefore, the pregnant women that had disclosed their HIV status displayed higher levels of depressive symptoms on the CES-D. Mixed results were illustrated in regard to HIV disclosure status, with HIV disclosure status being associated with higher scores on the CES-d but not on the EPDS. Application of Pearson $(r)$ correlation analyses allowed the researcher to determine the direction and strength of the relationship between variables (Penhazur \& Schmelkin, 1991). This statistical approach allowed the researcher to determine whether or not inherited vulnerabilities were significantly related to depressive symptoms (See Table 4.13). Pregnant women who disclosed their HIV status had significantly higher depression scores (more depressive symptoms) on the CES-D (mean 18.16) but not the EPDS (mean 8.95) compared to women who had not disclosed their status (mean CES-D score 5; mean EPDS score 4).

Sleep quality was measured using the PSQI, with a cut-off score of 5 or greater indicating poor sleep quality. In this group of pregnant women, there was a significant strong, positive correlation between sleep quality and depressive symptoms on the CES$\mathrm{D}(r=0.61 ; p=0.0004)$ and EPDS $(r=0.67 ; p=0.0001)$. As sleep quality scores increased (worsened), depression scores increased (worsened). Sleep quality was the acquired vulnerability with the strongest association with depressive symptoms in pregnant participants.

History of sexual abuse or assault was measured on the Demographic Questionnaire. Results indicated a low positive, but non-significant correlation between depressive symptoms and sexual abuse or assault when measured with the CES-D $(r=$ $0.30 ; p=0.23)$. Similarly, correlation was non-significant when the EPDS was used to assess depressive symptoms $(r=0.27 ; p=0.24)$. Thus, results of these analyses indicated that there was not a significant association between history of sexual assault or abuse and depressive symptoms when measured using both the CES-D and EPDS in this group of pregnant African American women who were HIV-positive.

The relationship of inherited vulnerabilities (e.g., family history of mood disorders and family history of alcohol and/or drug use) to depressive symptoms among non-pregnant African American women who were HIV positive was examined using correlation analyses. Pearson's correlations were estimated to answer this research question as most variables were normally distributed. The presence or absence of each of the inherited vulnerabilities was obtained in the Demographic Questionnaire.

The associations between inherited vulnerabilities and depressive symptoms were not significant in this group of non-pregnant women. Family history of mood disorders was dummy coded as 0 if there was no family history of mood disorders and 1 if there was a positive family history of mood disorders. The correlation between family history of mood disorders and depressive symptoms with the CES-D was not statistically 
Table 4.13 Pearson Correlation $(r)$ Analyses between Inherited Vulnerabilities and Depressive Symptoms for Non-Pregnant African American Women Who Were HIVpositive $(n=60)$.

\begin{tabular}{lcc}
\hline & $\begin{array}{c}\text { CES-D Depressive } \\
\text { Symptoms Scores } \\
r(p \text { value })\end{array}$ & $\begin{array}{c}\text { EPDS Depressive } \\
\text { Symptoms Scores } \\
r(p \text { value })\end{array}$ \\
\hline $\begin{array}{l}\text { Inherited Vulnerability } \\
\text { disorders }\end{array}$ & $.23(0.08)$ & $.23(0.08)$ \\
$\begin{array}{l}\text { Family history of } \\
\text { alcohol/drug use }\end{array}$ & $.10(0.46)$ & $.25(0.06)$ \\
\hline
\end{tabular}

Note: $\mathrm{CES}-\mathrm{D}=$ Center for Epidemiologic Studies Depression Scale; EPDS=Edinburgh Postnatal Depression Scale. A priori alpha for the study was $p \leq 0.05$. 
significant $(r=0.23, p=0.08)$. Similarly, there was a non-significant association $(r$ $=0.23, p=.08$ ) between family history of mood disorders and depressive symptoms when measured by the EPDS. Thus, family history of mood disorders was not significantly associated with depressive symptoms in this group of non-pregnant African American women who were HIV-positive.

Family history of alcohol and/or drug use was not significantly correlated with depressive symptoms when measured by the CES-D $(r=0.10 ; p=0.46)$, or the EPDS ( $r$ $=0.25 ; p=0.06$ ). Thus, family history of alcohol and/or drug use was not associated with depressive symptoms in this group of non-pregnant African American women who were HIV-positive when measured by the CES-D and EPDS. Participants who had a positive family history of alcohol and/or drug use reported greater depressive symptoms on the CES-D (mean CES-D score 20.18) and the EPDS (mean EPDS score 11.5) compared to women who did not have a positive family history (mean CES-D score 17.68; mean EPDS score 7.79).

The relationship of acquired vulnerabilities (educational level, income level, alcohol and/or drug use, HIV disclosure status, sleep quality, history of sexual abuse or assault) to depressive symptoms among non-pregnant African American women who were HIV positive was examined.

Educational level was captured on the Demographic Questionnaire in a categorical fashion. Although four categories were present on the questionnaire, the majority of the sample clustered in two categories, less than high school graduate or equivalent diploma (HS/GED) and high school graduate or equivalent diploma (HS/GED) or higher, therefore these two categories were used for correlation analysis. Dummy coding was used with a score of zero assigned for women with less than HS/GED and one for women who had HS/GED or higher educational levels. Educational level was not significantly correlated with depressive symptoms when measured with the CES-D $(r=0.17 ; p=0.19)$. or EPDS $(r=0.17 ; p=0.18)$ in non-pregnant study participants.

Income level was also captured on the Demographic Questionnaire as annual household income in a categorical fashion. Although there were five categories present, the sample responses primarily clustered in two categories, less than $\$ 5000$ per year and $\$ 5000$ per year or greater, thus these two categories were used for analysis. Dummy codes of 0 and 1 were assigned to signify income less than $\$ 5000$ per year and $\$ 5000$ per year or greater, respectively. Income level was not significantly correlated with depressive symptoms when measured with the CES-D $(r=0.14 ; p=0.29)$ or the EPDS $(r$ $=-0.04 ; p=0.76$ ). Thus, income level was not significantly associated with depressive symptoms in this group of non-pregnant African American women who were HIVpositive.

Current alcohol and/or drug use was captured on the Demographic Questionnaire. Prior to conducting the zero-order correlation analysis, substance use was dummy coded as zero if there was no history and one if there was a positive history of this 
characteristic. Athough substance use was an acquired vulnerability that was positively correlated with depressive symptoms, the strength of the association was weak but significant using the CES-D $(r=0.28 ; p=0.03)$, but not on the EPDS $(r=0.24 ; p=$ $0.06)$. Thus, substance use garnered mixed results; significantly associated with depressive symptoms on one of the two depressive symptom measurements in this group of non-pregnant African American women who were HIV-positive. Depression symptoms were significantly higher in non-pregnant women who had a positive history of substance use compared to women who did not report alcohol and/or drug use.

The number of persons the subject had disclosed her HIV status to on the Demographic Questionnaire reflected their HIV disclosure status. Dummy coding was implemented to signify two categories: no disclosure to others, coded as 0 , or disclosure to others, coded as 1 . HIV disclosure status was not significantly correlated with depressive symptoms when measured by the CES-D $(r=-0.15 ; p=0.24)$ or the EPDS ( $r$ $=-0.16 ; p=0.38)$ in this group of non-pregnant African American women who were HIV-positive.

Sleep quality was measured using the PSQI, with a cut-off score of 5 or greater suggestive of poor sleep quality. In this group of non-pregnant women, there was a significant positive correlation between sleep quality and depressive symptoms on the CES-D $(r=0.69 ; p<0.001)$ and EPDS $(r=0.63 ; p<0.001)$. The strength of both of these correlations was moderate. As sleep quality scores increased (e.g. poorer sleep quality), depressive symptoms scores significantly increased (more depressive symptoms occurred). Thus, sleep quality was significantly associated with depressive symptoms in this group of non-pregnant African American women who were HIV-positive.

History of sexual abuse or assault was measured on the Demographic Questionnaire. Responses were dummy coded as no history of sexual abuse or assault (coded as 0 ) or history of sexual abuse or assault (coded as 1). This acquired vulnerability was not significantly correlated with depressive symptoms on the CES-D $(r=0.24 ; p=$ $0.06)$ or the EPDS $(r=0.18 ; p=0.16)$. Thus, history of sexual assault was not significantly related to depressive symptoms in this group of non-pregnant African American women who were HIV-positive. Table 4.14 provides a summary of findings related to acquired vulnerabilities and depressive symptoms in this group of non-pregnant women.

\section{AIM THREE}

Examine the associations among inherited (family history of mood disorders, family history of alcohol and/or use) and acquired (educational level, income level, alcohol and/or drug use, HIV disclosure status, sleep quality, history of sexual abuse or assault) vulnerabilities and anxiety symptoms in pregnant and non-pregnant African American women who were HIV-positive. 
Table 4.14 Pearson Correlation ( $r$ ) Analysis between Acquired Vulnerabilities and Depressive Symptoms for Non-Pregnant African American Women Who Were HIVpositive $(n=60)$.

\begin{tabular}{lcc}
\hline Acquired Vulnerability & $\begin{array}{c}\text { CES-D Depressive } \\
\text { Symptoms Scores } \\
\mathrm{r}(p \text { value })\end{array}$ & $\begin{array}{c}\text { EPDS Depressive } \\
\text { Symptoms Scores } \\
\mathrm{r}(p \text { value })\end{array}$ \\
\hline Income & $.14(.29)$ & $-.04(.76)$ \\
Educational Level & $.17(.19)$ & $.17(.18)$ \\
Alcohol and/or Drug Use & $.28(.03)^{*}$ & $.24(.06)$ \\
HIV Disclosure Status & $-.15(.24)$ & $-.12(.38)$ \\
History of Sexual Assault & $.24(.06)$ & $.18(.16)$ \\
Sleep Quality & $.69(<.0001)^{*}$ & $.63(<.0001)^{*}$ \\
\hline
\end{tabular}

Note: $C E S-D=C e n t e r$ for Epidemiologic Studies Depression Scale; EPDS=Edinburgh Postnatal Depression Scale. The symbol $r$ refers to Pearson Correlation. A priori alpha for the study was $p \leq 0.05$. 
Pregnant African American women who were HIV-positive were assessed. The influence of inherited vulnerabilities (e.g. family history of mood disorders, family history of alcohol and/or drug use) on anxiety symptoms among pregnant African American women who were HIV positive was examined using correlation statistics.

Family history of mood disorders was dummy coded, with no family history of mood disorders coded as 0 and positive family history of mood disorders coded as 1 . Family history of mood disorders was significantly correlated with anxiety symptoms on the BAI ( $r=0.64 ; p=0.003)$. Thus, a positive family history of mood disorders was associated with greater anxiety scores in this group of pregnant African American women who were HIV-positive.

Family history of alcohol and/or drug use was captured on the Demographic Questionnaire. Results were dummy coded for categorization, with no family history of alcohol and/or drug use (coded as 0 ) and positive family history of alcohol and/or drug use (coded as 1). Family history of alcohol and/or drug use was not significantly correlated with anxiety symptoms in this group of pregnant women $(r=0.35 ; p=0.13)$. Table 4.15 provides an illustration of these results.

Correlation statistics were used to examine the association of acquired vulnerabilities (educational level, income level, alcohol and/or drug use, HIV disclosure status, sleep quality, history of sexual abuse or assault) to anxiety symptoms among pregnant African American women who are HIV positive.

Educational level was captured on the demographic questionnaire in a categorical fashion. As with the previous analyses, four categories were collapsed into two categories, less than HS/GED (coded as 0 ) and HS/GED or greater (coded as 1). Most of the participants reported educational levels in these categories. Educational level was negatively correlated with elevated anxiety symptoms when measured with the BAI however, the association was non-significant $(r=-0.6 ; p=0.81)$ in this group of pregnant African American women who were HIV-positive.

Income level was captured on the Demographic Questionnaire as annual household income in a categorical fashion. As with earlier analyses, five categories were collapsed into two, illustrating less than $\$ 5000$ per year (coded as 0 ) and $\$ 5000$ per year or greater (coded as 1). Income level was not significantly correlated with anxiety symptoms when measured be the BAI $(r=0.12 ; p=0.66)$ in this group of pregnant African American women who were HIV-positive.

Alcohol and/or drug use was captured on the Demographic Questionnaire. Dummy coding was implemented to signify no substance use (coded as 0 ) or positive substance use (coded as 1 ). Substance use had a positive but statistically non-significant correlation with anxiety scores on BAI $(r=0.25 ; p=0.3)$. 
Table 4.15 Pearson Correlation ( $r$ ) Analysis between Inherited Vulnerabilities and Anxiety Symptoms for Pregnant African American Women Who Were HIV-positive $(n=20)$.

\begin{tabular}{lc}
\hline Inherited Vulnerability & $\begin{array}{c}\text { Anxiety Symptom (BAI) Scores } \\
r(p \text { value })\end{array}$ \\
\hline Family History of Mood Disorders & $.64(.0003)^{*}$ \\
Family History of Alcohol/Drug Use & $.35(.13)$ \\
\hline
\end{tabular}

Note: BAI= Beck Anxiety Inventory. Study a priori alpha was ${ }^{*} p \leq .05$. 
Disclosure of HIV status was measured on the Demographic Questionnaire. Dummy coding was implemented to signify no disclosure to others (coded as 0 ) or disclosure to others (coded as 1). HIV disclosure status was positively and significantly correlated with anxiety symptoms when measured by the BAI $(r=0.52 ; p=0.02)$. Thus, pregnant HIV-positive African American women in the sample who disclosed their HIV status had significantly higher anxiety scores (more severe anxiety symptoms) than women who had not disclosed their status.

Sleep quality was measured using the PSQI, with a cut-off score of 5 or greater indicating poor sleep quality. In this group of pregnant women, there was a significant positive correlation between sleep quality and anxiety symptoms on the BAI $(r=0.68 ; p$ $=0.001$ ). Thus, women with greater sleep quality scores (poorer sleep quality) also had greater anxiety scores (more severe anxiety) in this group of pregnant African American women who were HIV-positive.

History of sexual abuse or assault was measured and responses were dummy coded as no history of sexual abuse or assault (coded as 0 ) or history of sexual abuse or assault (coded as 1). History of sexual abuse or assault was positively correlated with anxiety scores on the BAI $(r=0.33 ; p=0.16)$, but this finding was not statistically significant at the $p<0.05$ level. Thus, history of sexual abuse or assault was not significantly related to anxiety symptoms in this group of pregnant African American women who were HIV-positive. Table 4.16 illustrates a summary of the correlation results for this group of pregnant women who were HIV-positive.

Non-pregnant African American women who were HIV-positive women were assessed and associations between inherited vulnerabilities (e.g. family history of mood disorders, family history of alcohol and/or drug use) and anxiety symptoms examined using correlation analyses. Anxiety symptoms were measured using the BAI. Family history of mood disorders was dummy coded 1 or 0 , respectively if the characteristic was present or absent. Family history of mood disorders was positively correlated with anxiety symptoms $(r=0.33 ; p=0.0093)$. Non-pregnant women with a positive family history of mood disorders had significantly higher anxiety scores (mean BAI score 16.77) than women who did not have a family history of these disorders (mean BAI score 8.6).

Family history of alcohol and/or drug use was not correlated with anxiety symptoms $(r=0.19 ; p=0.14)$. Women who had a positive family history of alcohol and/or drug use reported more anxiety symptoms (mean BAI score 14.59) than women who had no family history (mean BAI score 9.86). Table 4.17 shows correlation results of inherited vulnerabilities with anxiety symptoms in the non-pregnant study participants, with family history of mood disorders being associated with higher BAI scores.

The influence of acquired vulnerabilities (e.g. educational level, income level, alcohol and/or drug use, HIV disclosure status, sleep quality, history of sexual abuse or assault) on anxiety symptoms among non-pregnant African American women who were HIV positive was examined using correlation analyses. 
Table 4.16 Pearson Correlation (r) Analysis between Acquired Vulnerabilities and Anxiety Symptoms for Pregnant African American Women Who Were HIV-positive $(n=20)$.

\begin{tabular}{cc}
\hline & $\begin{array}{c}\text { Anxiety Symptoms } \\
\text { (BAI Scores) } \\
r(p \text { value })\end{array}$ \\
Acquired Vulnerability & $.25(.30)$ \\
Alcohol and/or Drug Use & $.6(.81)$ \\
Educational Level & $.12(.66)$ \\
Income & $.52(.002)^{*}$ \\
HIV Disclosure Status & $.68(.0010)^{*}$ \\
Sleep Quality & $.33(.16)$ \\
\hline History of Sexual Assault
\end{tabular}

Note: BAI=Beck Anxiety Inventory. Study a priori alpha was ${ }^{*} p \leq .05$. 
Table 4.17 Pearson Correlation ( $r$ ) Analysis between Inherited Vulnerabilities and Anxiety Symptoms for Non-Pregnant African American Women Who Were HIVpositive $(n=60)$.

\begin{tabular}{lc}
\hline & $\begin{array}{c}\text { Anxiety Symptom } \\
\text { (BAI) Scores } \\
\text { Inherited Vulnerability }\end{array}$ \\
\hline Family History of Mood Disorders & $.33(.0093)^{*}$ \\
Family History of Alcohol/Drug Use & $.19(.14)$ \\
\hline
\end{tabular}

Note: BAI $=$ Beck Anxiety Inventory. A priori alpha was ${ }^{*} p \leq .05$. 
Educational level was captured in a categorical fashion. Most of the sample fell into two main categories: less than HS/GED or HS/GED or greater, the four categories were collapsed into these two categories for statistical analysis. Educational level was not significantly correlated with anxiety symptoms when measured with the BAI in this group of non-pregnant women who were HIV positive $(r=0.1 ; p=0.44)$.

Income level was captured in a categorical fashion on the Demographic Questionnaire as annual household income. Two main categories of income were present in the sample. Dummy coding was implemented to signify income less than $\$ 5000$ per year (coded as 0 ) and $\$ 5000$ per year or greater (coded as 1). Income level was not significantly correlated with anxiety symptoms when measured with the BAI $(r=0.21 ; p$ $=0.12$ ) in this group of non-pregnant African American women who were HIV-positive.

Substance use was coded 0 if the characteristic was absent and 1 if present. Substance use had a positive correlation with anxiety scores on BAI $(r=0.24)$. The strength of this correlation was weak, and was not statistically significant $(p=$ 0.06).Thus, a history of substance use was not significantly associated with higher anxiety scores for this group of non-pregnant women.

Disclosure of HIV status was reported on the Demographic Questionnaire as the number of persons to whom the participant had disclosed her HIV status. Dummy coding was implemented to signify no disclosure to others as a score of 0 or disclosure to others as a score of 1 . HIV disclosure status was not significantly correlated with anxiety symptoms when measured by the BAI $(r=-0.0072 ; p=0.96)$ in this group of nonpregnant African American women who were HIV-positive.

Sleep quality was measured using the PSQI, with a cut-off score of 5 or greater indicating poor sleep quality. In this group of non-pregnant women, there was a significant positive correlation between sleep quality and anxiety symptoms on the BAI $(r=0.69 ; p<.0001)$. Thus poorer sleep quality was significantly associated with more anxiety symptoms in this group of non-pregnant women.

History of sexual abuse or assault was measured on the Demographic Questionnaire. Responses were dummy coded as no history of sexual abuse or assault (coded as 0 ) or history of sexual abuse or assault (coded as1). A history of sexual abuse or assault was not significantly correlated with anxiety scores on the BAI $(r=0.15 ; p=$ 0.26 ) in this group of non-pregnant African American women who were HIV-positive. Table 4.18 provides a summary of these correlation analyses.

\section{AIM FOUR}

Examine the influence of inherited vulnerabilities (family history of mood disorders, family history of alcohol and/or drug use) and acquired vulnerabilities (educational level, income status, alcohol and/or drug use, HIV disclosure status, sleep quality, 
Table 4.18 Pearson Correlation ( $r$ ) Analysis between Acquired Vulnerabilities and Anxiety Symptoms for Non-Pregnant African American Women Who Were HIVpositive $(n=60)$.

\begin{tabular}{lc}
\hline & $\begin{array}{c}\text { Anxiety Symptoms (BAI Scores) } \\
\text { Acquired Vulnerability }\end{array}$ \\
\hline Alcohol and/or Drug Use & $.24(.06)$ \\
Educational Level & $.10(.44)$ \\
Income & $.21(.12)$ \\
HIV Disclosure Status & $-.01(.96)$ \\
Sleep Quality & $.69(<.0001)^{*}$ \\
History of Sexual Assault & $.15(.26)$ \\
\hline
\end{tabular}

Note: BAI=Beck Anxiety Inventory. Study a priori alpha was * $p \leq .05$. 
history of sexual abuse or assault) on depressive symptoms in non-pregnant African American women who were HIV-positive.

In the current study, variables were identified as appropriate for entry as predictors into the multiple regression model through the use of Pearson correlation coefficients. Pearson correlation coefficients were estimated between dependent variables (depressive symptoms as measured by total CES-D scores) and independent variables (inherited and acquired vulnerabilities). Statistically significant correlations were identified as those with a $p$ value $\leq 0.2$. Only one independent inherited variable, family history of mood disorder met this criterion and was entered into the stepwise equation. Stepwise multiple regression analyses with backward elimination were performed and variables were eliminated if the variable was $\geq .20 p$-value. In stepwise multiple regression analyses, all independent variables were entered one by one into the regression model, and then one by one, each variable is deleted in order to identify a significant drop in $R^{2}$ (Penhazur \& Schmelkin, 1991). The stepwise approach with backward elimination was selected in order to illustrate the power of a single independent variable on the dependent variables (depressive and anxiety symptoms).

For regression analysis, family history of mood disorders was the only inherited variable that remained in the model. However, neither the model, nor the predictor, family history of mood disorders, was statistically significant. Only $4 \%$ of the adjusted variance in depressive symptom scores was explained by a positive family history of mood disorder. Table 4.19 illustrates simple regression analysis for depressive symptoms as measured by CES-D scores in this sample of non-pregnant women.

The same statistical analyses were conducted to examine the influence of inherited vulnerabilities on depressive symptoms as measured by total EPDS scores. Two variables were entered into the stepwise equation (family history of mood disorders and family history of alcohol and/or drug use) because Pearson correlation coefficients were significant at the $\leq .20$ level. Backward elimination was performed and independent variables were eliminated if the individual variable was $\geq .20 p$-value. The only variable eliminated from the model was family history of mood disorder. The final model retained family history of substance use; however, neither the predictor nor the model was statistically significant. Only $5 \%$ of the variance in depressive symptom scores was explained by a positive family history of substance use. Table 4.20 illustrates the complete simple regression results for the influence of inherited vulnerabilities on depressive symptoms as measured by the EPDS.

Based on the zero-order correlations, the acquired vulnerabilities (educational level, income level, alcohol and/or drug use, HIV disclosure status, sleep quality, history of sexual abuse or assault) were entered into the first regression model conducted to predict depressive symptoms using the CES-D.

Four variables were entered into the stepwise equation (educational level, alcohol and/or drug use, sleep quality, and history of sexual abuse or assault) as they met the previously described selection criteria. Two variables were eliminated from the model, 
Table 4.19 Summary of Stepwise Simple Regression Analysis by Backward Elimination for Depressive Symptom Scores Using the CES-D* among NonPregnant African American HIV-positive Women $(n=60)$.

\begin{tabular}{lcccc}
\hline & & Parameter & & \\
& Predictor & Estimate & $S E$ & $p$ \\
\hline Intercept & 16.421053 & 1.995271 & 8.23 & $<.0001$ \\
Family History of Mood & 5.9425837 & 3.295081 & 1.80 & 0.0765 \\
Disorders & & & \\
\hline
\end{tabular}

Note. $R^{2}=0.0531$; adjusted $R^{2}=0.036774, d f=59$; CES-D $=$ Center for Epidemiologic Studies Depression Scale. Predictor variable classified as an inherited vulnerability 
Table 4.20 Summary of Stepwise Simple Regression Analysis by Backward Elimination for Depressive Symptoms Measured by EPDS* among Non-Pregnant African American HIV-positive Women $(n=60)$.

\begin{tabular}{lcccc}
\hline & Parameter & & & \\
Predictor & Estimate & $S E$ & $\mathrm{t}$ & $p$ \\
\hline Intercept & 7.78947 & 1.155712 & 6.74 & $<.0001$ \\
Family History of & 3.7105263 & 1.908595 & 1.94 & 0.0567 \\
Alcohol/Drug Use & & & & \\
\hline
\end{tabular}

Note. $R^{2}=0.0612$, adjusted $R^{2}=0.045, d f=58$;

*EPDS= Edinburgh Postnatal Depression Scale. Predictor variable is classified as an inherited vulnerability 
educational level and history of sexual abuse or assault. The final model retained substance use and sleep quality and both predictors as well as the model were statistically significant. Substance use was significant at the $p=.0020$ level, indicating that a positive history of substance abuse was significantly associated with higher depressive symptom levels on the CES-D. Sleep quality was significant at the $p<.0001$ level, indicating that poorer sleep quality was significantly associated with higher depressive symptom levels on the CES-D. Thus, both predictors and the model were significant (Model statistics $R^{2}$ $=.5451, d f=57, p<.0001$ ). Greater depressive symptoms were associated with a history of substance use and poorer sleep quality. Table 4.21 provides a summary of multiple regression analysis results.

Based on the zero-order correlations, the acquired vulnerabilities (educational level, income level, alcohol and/or drug use, HIV disclosure status, sleep quality, history of sexual abuse or assault) were entered into the second regression model. Stepwise multiple regression with backward elimination was conducted to predict depressive symptoms using the EPDS. Three variables were entered into the stepwise equation (substance use, educational level, and sleep quality). The only variable eliminated from the model was educational level. The final model retained substance use and sleep quality and both predictors and the model were statistically significant. Substance abuse was significant at the $p=.0145$ level, indicating that a positive history of substance abuse was significantly associated with higher depressive levels on the EPDS total score. Sleep quality was significant at the $p<.0001$ level, indicating that poorer sleep quality was significantly associated with higher depressive symptom levels on the EPDS total score. Acquired vulnerabilities accounted for $46 \%$ of the variance in EPDS total score. The regression model for depressive symptoms indicated that substance use and sleep quality were the significant acquired vulnerabilities that influenced the EPDS total score. In contrast, education level was not a significant predictor of depression. See Table 4.22 for complete model results.

\begin{abstract}
AIM FIVE
Examine the influence of inherited vulnerabilities (family history of mood disorders, and family history of alcohol and/or drug use) and acquired vulnerabilities (educational level, income level, alcohol and/or drug use, HIV disclosure status, sleep quality, and history of sexual abuse or assault) on anxiety symptoms in non-pregnant African American women who are HIV-positive. Although anxiety symptom levels were relatively low, regression analyses were completed for prediction purposes.

Stepwise multiple regression analysis with backward elimination was used to answer this question. In order to identify the most appropriate variables for entry as predictors into the regression model, Pearson correlation coefficients were estimated between outcome variables and predictor variables. Using the bivariate results obtained in correlation analyses, separate multiple regression analyses with backward elimination were conducted for inherited and acquired vulnerabilities with the outcome variable (BAI
\end{abstract}


Table 4.21 Summary of Stepwise Multiple Regression Analysis by Backward Elimination for Depressive Symptoms Measured by CES-D among Non-Pregnant African American HIV-positive Women $(n=60)$.

\begin{tabular}{lcccc}
\hline & Parameter & & & \\
Predictor & Estimate & $S E$ & $t$ & $p$ \\
\hline Intercept & 2.3882439 & 2.201078 & 1.09 & 0.2825 \\
Alcohol and/or Drug Use & 7.199529 & 2.227402 & 3.23 & 0.0020 \\
Sleep Quality & 1.7276818 & 0.218227 & 7.92 & $<0.0001$ \\
\hline
\end{tabular}

Note. $\mathrm{R}^{2}=.5731$, Adjusted $\mathrm{R}^{2}=.5420, d f=55$. Predictor variables are classified as acquired vulnerabilities. 
Table 4.22 Summary of Stepwise Multiple Regression Analysis by Backward Elimination for Depressive Symptoms Measured by EPDS among Non-Pregnant African American HIV-positive Women $(n=60)$.

\begin{tabular}{lcccc}
\hline & Parameter & & & \\
Predictor & Estimate & $S E$ & $t$ & $p$ \\
\hline Intercept & 0.6813078 & 1.428049 & 0.48 & 0.6351 \\
Alcohol and/or Drug Use & 3.6451532 & 1.445128 & 2.52 & 0.0145 \\
Sleep Quality & 0.9085051 & 0.141584 & 6.42 & $<0.0001$ \\
\hline
\end{tabular}

Note. $R^{2}=.46$, Adjusted $R^{2}=.4344, d f=56$. Predictor variables classified as acquired vulnerabilities. EPDS = Edinburgh Postnatal Depression Scale. 
scores to assess anxiety).

Two inherited vulnerabilities were entered into the stepwise equation, family history of mood disorders and family history of substance use, because Pearson correlation coefficients were significant at the $\leq .20$ level. Backward elimination was performed and variables were eliminated if the individual variable was $\geq .20 p$-value. The only variable eliminated from the model was family history of substance use. The final model retained family history of substance use, which was non-significant $(p=.1402)$. Table 4.23 depicts complete multiple regression results for this model.

The influence of acquired vulnerabilities on anxiety symptoms among nonpregnant African American women who were HIV-positive was examined using stepwise multiple regression analysis with backward elimination. In order to identify which predictors could be used for regression analyses, Pearson correlation coefficients were estimated between outcome variables and acquired vulnerabilities. Statistically significant correlations were identified, and these items were used in the multiple regression analysis. Although there were six hypothesized predictor variables identified as potential acquired vulnerabilities, only alcohol and/or drug use and sleep quality were significantly correlated with anxiety and included in the regression analyses.

Backward elimination was performed and variables were eliminated if the individual variable was $\geq .20 \mathrm{p}$-value. Neither variable was eliminated from the model. Substance use was significant at the $p=.0073$ level, indicating that a positive history of alcohol and/or drug use was significantly associated with higher anxiety symptom levels on the BAI. Sleep quality was significant at the $p \leq 0.05$ level, indicating that poorer sleep quality was significantly associated with higher anxiety symptom levels as measured by the BAI. Thus, both predictors and the model were significant (Model statistics $\left.R^{2}=.5259, F=.33 .72, d f=2, p<.0001\right)$. Thus, greater anxiety symptoms were associated with alcohol and/or drug use and poorer sleep quality. Table 4.24 provides a summary of the model statistics.

Regression diagnostic analyses were performed to evaluate bivariate relationships between predictor variables included in the multiple regression analyses. The use of highly intercorrelated variables should be avoided, as their influence on one another limits individual predictor strength. Schroeder (Schroeder, 1990) and Fox (Fox, 1997) provide suggestions regarding identification of multicollinearity. For each predictor variable in the regression model, correlation coefficients should be identified. Potential problems may exist if there is a high correlation coefficient that exists between variables. Schroeder (Schroeder, 1990) and Fox (Fox, 1997) also suggest using methods such as estimating tolerance to test the validity of regression models. Variance inflation factor analysis is also useful to detect multicollinearity. A tolerance close to zero is indicative of collinearity; a variance inflation factor $<10$ is indicative of no significant collinearity. For the current study a tolerance level less than .20 and variance inflation factor greater than 4.0 were indicative of significant collinearity. 
Table 4.23 Summary of Stepwise Multiple Regression Analysis by Backward Elimination for Anxiety Symptoms Measured by BAI among Non-Pregnant African American HIV-positive Women $(n=60)$.

\begin{tabular}{lcccc}
\hline & Parameter & & & \\
Predictor & Estimate & $S S$ & $F$ & $p$ \\
\hline Intercept & 8.10954 & 0 & 0.00000 & 1.0000 \\
Family History of Mood & 7.42638 & 672.1055 & 5.187 & $0.0265^{*}$ \\
$\begin{array}{l}\text { Disorders } \\
\text { Family History of }\end{array}$ & 2.09305 & 53.38781 & 0.412 & 0.5235 \\
Alcohol/Drug Use & & & & \\
\hline
\end{tabular}

Note. $R^{2}=0.1174$, adjusted $R^{2}=0.0865, d f=57, p=.1402$. Predictor variables are classified as inherited vulnerabilities. BAI $=$ Beck Anxiety Inventory 
Table 4.24 Summary of Stepwise Multiple Regression Analysis by Backward Elimination for Anxiety Symptoms Measured by BAI among Non-Pregnant African American HIV-positive Women $(n=60)$.

\begin{tabular}{lcccc}
\hline & Parameter & & & \\
Predictor & Estimate & $S S$ & $F$ & $p$ \\
\hline Intercept & -3.46575 & 0.000 & 1.000 & \\
Alcohol and/or Drug Use & 6.01172 & 520.394 & 7.739 & 0.0073 \\
Sleep Quality & 1.64074 & 4038.277 & 60.051 & 0.0000 \\
\hline
\end{tabular}

Note. $\mathrm{R}^{2}=.5420$, Adjusted $\mathrm{R}^{2}=.5259, d f=57, p<.0001$ Predictor variables are classified as acquired vulnerabilities. BAI $=$ Beck Anxiety Inventory 
For each predictor variable used in this study, tolerance and variance inflation factor was examined. Tables 4.25 through 4.28 illustrate regression diagnostic analyses suggesting that multicollinearity was present at acceptable levels in the study models. 
Table 4.25 Collinearity Diagnostics for the Model of Inherited Vulnerabilities and Anxiety Symptoms $(n=60)$.

\begin{tabular}{lccc}
\hline & & & Variance Inflation \\
Variable & $d f$ & Tolerance & Factor \\
\hline Intercept & 1 & & 0.0000 \\
Family History of Mood Disorders & 1 & .8898 & 1.2385 \\
Family History of Alcohol/Drug Use & 1 & .8898 & 1.2385 \\
\hline
\end{tabular}

Note. Tolerance levels less than .20 and variance inflation factor levels greater than 4.0 indicative of significant collinearity. 
Table 4.26 Collinearity Diagnostics for the Model of Acquired Vulnerabilities and Anxiety Symptoms $(n=60)$.

\begin{tabular}{lccc}
\hline & & & Variance Inflation \\
Variable & $d f$ & Tolerance & Factor \\
\hline Intercept & 1 &. & 0.0000 \\
Alcohol and or Drug Use & 1 & .9999 & 1.0001 \\
Sleep Quality & 1 & .9999 & 1.0001 \\
\hline
\end{tabular}

Note. Tolerance levels less than .20 and variance inflation factor levels greater than 4.0 indicative of significant collinearity. 
Table 4.27 Collinearity Diagnostics for the Model of Depression as Measured with the Center for Epidemiologic Depression Scale and Acquired Vulnerabilities $(n=60)$.

\begin{tabular}{lccc}
\hline & & & Variance Inflation \\
Variable & $d f$ & Tolerance & Factor \\
\hline Intercept & 1 & & .0000 \\
Alcohol and or Drug Use & 1 & .9360 & 1.068444 \\
Education Level & 1 & .9689 & 1.0321 \\
Sleep Quality & 1 & .94503 & 1.0582 \\
History of Sexual Assault & 1 & .9001 & 1.1109 \\
\hline
\end{tabular}

Note. Tolerance levels less than .20 and variance inflation factor levels greater than 4.0 indicative of significant collinearity. 
Table 4.28 Collinearity Diagnostics for the Model of Caregiver Depression as Measured with the Edinburg Postnatal Depression Scale and Acquired Vulnerabilities $(n=60)$.

\begin{tabular}{lccc}
\hline & & & Variance Inflation \\
Variable & $d f$ & Tolerance & Factor \\
\hline Intercept & 1 &. & .0000 \\
Alcohol and or Drug Use & 1 & .9979 & 1.0021 \\
Education Level & 1 & .9786 & 1.0219 \\
Sleep Quality & 1 & .9806 & 1.0198 \\
\hline
\end{tabular}

Note. Tolerance levels less than .20 and variance inflation factor levels greater than 4.0 indicative of significant collinearity. 


\section{CHAPTER 5. DISCUSSION AND IMPLICATIONS}

\section{DISCUSSION}

The systematic investigation of predictors of depressive and anxiety symptoms among African American HIV-positive women has been understudied. Thus, the aims of this study were to identify the levels of depressive and anxiety symptoms and the predictors of depressive and anxiety symptoms in African American women who were HIV-positive and receiving health care in a mid-southern city in the United States. While preliminary, these findings provide direction that can help health care providers recognize HIV-positive women who are at increased risk for the development of mood disorders.

\section{DEMOGRAPHIC FINDINGS}

The majority of the women in the present study were poor and unmarried. Most participants reported their marital status as single ( $n=14,70 \%$ pregnant vs. $n=32,53.3 \%$ non-pregnant). A few of the non-pregnant group $(n=6,10 \%)$ were widowed. One woman reported AIDS as the cause of her spouse's death; another woman reported that her husband committed suicide after she was hospitalized and diagnosed with AIDS. This participant also reported that after the suicide, she learned that her husband knew about his HIV diagnosis for many years prior to her diagnosis.

Almost half (49\%) of the total sample of pregnant and non-pregnant women reported annual household incomes of less than $\$ 5000,(63 \%$ of the pregnant women; $44 \%$ of the non-pregnant women). Almost one-half (47.3\%) of the total sample was unemployed, whereas $45 \%$ of the total sample reported part-time employment. Levels of disability were not assessed for this sample of women; however disability could explain the high prevalence of unemployment reported by study participants. These results are similar to findings of regional HIV demographic results, indicating that HIV is a disease that significantly affects southern, minority women who are poor (Centers for Control and Prevention, 2007; Centers for Disease Control and Prevention, 2007b, 2007c; Reif, Whetten, Ostermann, \& Raper, 2006).

Levels of disability were not assessed for this sample of women; however disability could explain the high prevalence of unemployment reported by study participants. These results are similar to findings of regional HIV demographic results, indicating that HIV is a disease that significantly affects southern, minority women who are poor (Centers for Disease Control and Prevention, 2005, 2007b, 2007c; Reif et al., 2006; U. S. Census Bureau, 2006). Participants in the current study were recruited from ambulatory clinics located within a public safety net hospital, adding to the likelihood that the sample would consist of predominately poor women. 


\section{AIM ONE. LEVELS OF DEPRESSIVE AND ANXIETY SYMPTOMS}

\section{Depressive Symptoms}

Scores on the CES-D and EPDS defined depressive symptoms in the current study. Although most participants denied feeling depressed in the two weeks prior to completion of the questionnaires, approximately $50 \%$ of participants in the sample had both CES-D and EPDS scores requiring referral to the social worker (e.g. case manager). Denial of depressive symptoms could be related to the fear and stigma of mental illness that is pervasive within many African American communities. Sussman and colleagues (Sussman, Robins, \& Earls, 1987) found that the proportion of African Americans who feared mental health treatment was 2.5 times greater than the proportion of whites. Research indicates that African Americans are more likely to be diagnosed with severe mental illnesses like paranoid schizophrenia and less likely to be diagnosed with affective disorders than their white counterparts (Adebimpe, 1981; Bolden \& Wicks, 2005; Neighbors et al., 1989). Stigma contributes to delays in seeking mental health care and treatment. Moreover, African Americans are more likely to use non-psychiatric services such as prayer, primary care, and emergency services, rather than seek specific mental health treatment (United States Department of Health and Human Services, 1999; K. Wells et al., 2001)

The clinically relevant depressive symptom scores found in this sample could reflect the overlap seen between symptoms of depression and symptoms of chronic illness. Overlapping symptoms for depression and HIV include poor appetite, weight loss, fatigue, and poor sleep (Kalichman, Rompa, \& Cage, 2000). The CES-D captures depressed affect, positive affect, and somatic complaints, however, researchers have illustrated similar factors in HIV-symptomatic persons (Cochran \& Mays, 1994), which may have resulted in inflated depressive symptoms scores on the CES-D in symptomatic HIV-positive persons (Kalichman et al., 2000). The mean CD4 count in this sample was over 500, reflecting a clinically stable population, although HIV specific symptoms were not assessed in this study. Some of the women who had elevated depressive symptom scores may have been clinically depressed and were referred for further evaluation. Two of the women were immediately assessed by their provider and started on antidepressants the day of their participation. Researchers have found similar rates of depressive symptoms in HIV positive women (Ickovics et al., 2001; McDaniel, Fowlie, Summerville, Farber, \& Cohen-Cole, 1995) .

The non-pregnant group of women in the present study had a higher level of depressive symptoms than the pregnant group of women. These results may be attributed to the fact that the non-pregnant group had a longer duration of HIV diagnoses. While this longer duration could provide time to accept the diagnosis, many of these women experienced multiple illness-related life events that potentially contributed to higher depressive symptom levels. Specifically, this group of non-pregnant women more often reported losing partners to AIDS, which may account for higher levels of depressive symptom scores. Pregnant women in the study might have felt greater hope and optimism than the non-pregnant group. In a meta-analysis of qualitative research (Sandelowski \& 
Barroso, 2003), HIV-positive women reported that motherhood gave them a sense of identity, reason to improve their lives, strength, hope, and joy. However these qualitative results do not account for the greater depressive symptoms reported by non-pregnant participants who were also mothers. HIV-positive mothers have reported negative impacts of their disease, specifically the stigma associated with the disease, negative impact of HIV on their children, and guilt associated with possible transmission of HIV to their children (Sandelowski et al., 2003). HIV-positive women have also reported a fear of disapproval from others when they decide to have children (Sandelowski et al., 2003). In the current study, pregnant participants' depressive symptoms scores could have been influenced by the hope that their unborn babies would not be HIV-infected; however this information was not elicited from the study sample.

\section{Anxiety Symptoms}

Although depressive and anxiety symptoms often occur simultaneously in the general population, the present study did not confirm the coexistence of clinically relevant levels of both symptom types. The levels of depressive symptoms were clinically relevant in both groups of women, while anxiety symptoms were in general uncommon in both groups. Most $(83 \%)$ of the sample had BAI scores of less than 22, which is indicative of little or no anxiety (A. T. Beck et al., 1988). Only three women $(15 \%)$ in the group of 20 pregnant women had BAI scores at or above a clinically relevant level compared to eight non-pregnant women (13\%). Conversely, almost half of the total sample of 80 women had CES-D scores (52.46\%) and EPDS scores (48.75\%) indicating the need for referral and additional assessment of depressive symptoms.

National data related to the co-morbidity of depressive and anxiety symptoms in the general population (Hirschfeld, 2001; R. C. Kessler, McGonagle, Nelson et al., 1994) indicate higher incidence rates than reported in the current sample of women. Kessler and colleagues (R. C. Kessler, McGonagle, Nelson et al., 1994) reported a 10.3\% prevalence rate for depression, while the prevalence rate for generalized anxiety disorder was $3.1 \%$. In a second larger study ( $N=9282)$, Kessler and colleagues (R. C. Kessler et al., 2005) reported anxiety as the most prevalent DSM-IV disorder in the general population (18.1\%), with over half of the participants having more than one disorder. In another study, $75 \%$ of patients diagnosed with major depressive disorder in the primary care setting also had an anxiety disorder (Olfson et al., 2000). Thus, the prevalence rate of anxiety symptoms in the current study was lower than that of the general population. A possible explanation for this finding is the underreporting of symptoms of anxiety. Evidence suggests that persons are likely to underreport symptoms that they perceive as embarrassing (R. C. Kessler et al., 2002; Merikangas et al., 1998). While these findings were unexpected, it is unclear if the low levels of anxiety symptoms occurred because women with significant depressive symptoms did not have clinical depression. Anxiety typically precedes depression (Zimmerman \& Chelminski, 2003). This point is particularly important as the depressive symptom instruments used in this study are screening tests rather than diagnostic tests. Clinically significant depressive symptom scores on screening tests may reflect a false positive score which is especially common in populations in which chronic illness symptoms overlap with symptoms of depression 
(Cavanaugh, Clark, \& Gibbons, 1983). Although the current study revealed low levels of anxiety symptoms in the presence of clinically important depressive symptoms, the continued assessment of both anxiety and depression is warranted due to national trends documenting their coexistence (R. C. Kessler et al., 2005).

\section{AIM TWO. ASSOCIATIONS AMONG INHERITED AND ACQUIRED VULNERABILITIES AND DEPRESSIVE SYMPTOMS}

Inherited Vulnerabilities and Depressive Symptoms

Non-pregnant women in the study sample with a positive family history of mood disorders, an inherited vulnerability, did not report associated increases in depressive symptom scores as measured by the CES-D or the EPDS. Similarly, non-pregnant women in the study sample with a positive family history of alcohol and/or drug use, an inherited vulnerability, did not report associated increases in depressive symptom scores as measured by the CES-D or the EPDS. Of the 80 women participating in this study only 26 reported a positive family history of alcohol and/or drug use. Additionally, the use of dummy-coding that was implemented for the categorization of family history results (presence or absence of the variable) limited the variability needed to show significant correlations, as the value for the score was either 0 or 1 .

Inherited vulnerabilities revealed similar mixed associations with depressive symptom scores in the group of pregnant women. Family history of mood disorders was significantly and positively associated with higher levels of depressive symptoms on both the CES-D and EPDS scores, however, no association was found between family history of alcohol and drug use and depressive symptom scores in this group of 20 pregnant women. These findings were surprising given that research in the general population has consistently indicated a link between depressive symptoms and a positive family history of both mood disorders and alcohol/drug use (Gutman \& Nemeroff, 2003; King et al., 2006; Kosten et al., 1992; Weissman et al., 2005). Alternative explanations for these findings may be denial of family history of alcohol and/or drug use or embarrassment, which could have lead to underreporting of family histories of alcohol and/or drug use. Of the 80 women participating in this study only 26 reported a positive family history of alcohol and/or drug use; a similar number $(n=28)$ reported a family history of mood disorder. For a correlation to be found between variables that are scored as 0 or 1 (the presence or absence of the variable of interest) there must be sufficient numbers of persons with and without the variable of interest to detect this association.

Acquired Vulnerabilities and Depressive Symptoms

Women who reported a positive history of personal alcohol and/or drug use and poor sleep quality as indicators of acquired vulnerabilities had higher levels of depressive symptoms on both the CES-D and EPDS, in both groups of women (pregnant and nonpregnant). These findings are consistent with the literature, with correlations between 
alcohol and drug use and depressive symptoms reported in women (Darko, McCutchan, Kripke, Gillin, \& Golshan, 1992; Darko, Mitler, \& Miller, 1998; Dixit \& Crum, 2000; Mock et al., 2002; Norman et al., 1992; Penzak, Reddy, \& Grimsley, 2000; Reardon \& Buka, 2002; Schutte et al., 1995; Tam, Weisner, \& Mertens, 2000; K. Wells et al., 2001), and African American populations (Reardon \& Buka, 2002; Tam et al., 2000; K. Wells et al., 2001).

Poor sleep quality and depressive symptoms have also been well-documented in the literature (Neubauer, 2004). Clinically relevant depressive symptom scores were found on the CES-D and EPDS in both groups (non-pregnant and pregnant) of women that had PSQI scores above the cut-off $(\geq 5)$. Most all of the women with clinically relevant PSQI scores had scores above the study mandated cutoffs for both the CES-D and EPDS. Results of the current study largely parallel published research with regard to poor sleep quality and depressive symptoms in HIV-positive women (Lee et al., 2001) and HIV-positive men (Perkins et al., 1994). Only seven women in the sample had clinically relevant PSQI scores without clinically relevant CES-D or EPDS scores. This strong association between sleep quality and depressive symptoms however could reflect sleep difficulties related to HIV, side effect of medications, or poor sleep environment (Darko et al., 1992; Darko et al., 1998; Mock et al., 2002; Norman et al., 1992; Penzak et al., 2000; Reardon \& Buka, 2002; Tam et al., 2000; K. Wells et al., 2001). Although causal relationships are difficult to infer, poor sleep quality and depressive symptoms are often concomitant problems, with one contributing to the other.

HIV disclosure status was significantly correlated with higher depressive symptom scores on the CES-D, but not the EPDS, in the group of pregnant women. The CES-D has a much wider scoring range than the EPDS, which may make it easier to find a significant association between depressive symptom levels and HIV disclosure status. When diagnostic criteria for depression according the DSM-IV are compared to the screening content of the CES-D and EPDS, both instruments target the same symptoms.

The clinically relevant depressive symptom scores on the EPDS in the population of pregnant women that had disclosed their HIV status was surprising. This finding was unexpected as the act of disclosure was hypothesized to decrease depressive symptoms, increase support services and networks, thereby decreasing depressive symptoms (Schmidt \& Goggin, 2002; D. K. Smith et al., 1996). However, Schmidt and Goggin (Schmidt \& Goggin, 2002) reported that the HIV-positive single mothers in their study reported less satisfaction from the disclosure process, lower levels of perceived social support, and higher levels of depressive symptoms, findings that were different than the majority of positive results associated with disclosure. Moreover, the majority of the sample in the current study was single and mothers, offering support to Schmidt and Goggin's (Schmidt \& Goggin, 2002) results, as well as a plausible explanation for these findings.

Additional alternative reasons for inconsistent results include the small sample size (which may not adequately reflect the experiences of the population) and the feelings of shame and abandonment that could have been felt by those women that had exposed 
their HIV to others. HIV is a disease that is not viewed by others with sympathy and support unlike other diseases such as cancer. When a disease, such as HIV, is frequently acquired through behavioral choices, the resulting perception may be that the person "deserves" the disease. If this is the perception, psychological and social support may be withheld. These confounding findings are noteworthy. Sensitivity of instrumentation should be evaluated when instruments designed to measure the same variable reveal different results. Mosack and Shore (Mosack \& Shore, 2003) reported that the CES-D identified higher numbers of women with depressive symptoms than the EPDS when administered to the same group of women. Although the family history of the sample in Mosack and Shore's study (Mosack \& Shore, 2003) was not discussed, the affect of family history cannot be ignored, and could have impacted their study results.

\section{AIM THREE. ASSOCIATIONS AMONG INHERITED AND ACQUIRED VULNERABILITIES AND ANXIETY SYMPTOMS}

\section{Inherited Vulnerabilities and Anxiety Symptoms}

Women in the present study $(N=80)$ who reported inherited vulnerabilities of family history of mood disorders had higher BAI scores than women who did not report family history of mood disorders. Similar findings were noted for women who reported a positive history of family history of alcohol and/or drug use, with their BAI scores higher than the women who did not report a family history of alcohol and/or drug use. Similar findings are reported in the literature. McLeod and colleagues (McLeod, Foster, HoehnSaric, Svikis, \& Hipsley, 1994) investigated the relationship between family history of alcoholism and increased levels of anxiety symptoms in a group of women that were being treated for generalized anxiety disorder $(n=54)$. The majority of the sample $(76 \%)$ reported an alcohol use disorder in a first degree relative. Investigators specified that within the group of women that reported a family history of alcohol use disorder, the majority of them (74\%) reported paternal family history. The current study did not specify which relative had a history of alcohol and/or drug use. Determining maternal or paternal history, as well as multi-generational information would have added depth to the family history aspect of the current study.

Similarly, anxiety disorders were prevalent among subjects $(N=454)$ with a family history of alcohol dependence in a longitudinal study of college students (Kushner, Sher, \& Erickson, 1999). Additional literature reflects that children born to parents with a history of substance use disorders have been shown to be at greater risk for developing drug and alcohol abuse problems of their own, as well as problems with anxiety and depression (Barnard \& McKeganey, 2004; Clark, Cornelius, Wood, \& Vanyukov, 2004; S. L. Harter, 2000). Family history of alcohol and/or drug use revealed inconsistent associations, with elevated BAI scores noted in the group of non-pregnant women but not the pregnant group. Alternative explanations for the confounding results could be a result of participants' inability to accurately recall family histories or possible reluctance to report potentially embarrassing family histories. 
Acquired Vulnerabilities and Anxiety Symptoms

The findings indicated that the women, both pregnant and non-pregnant, who reported active substance use and poor sleep quality, had elevated BAI scores. Similar results are noted in published studies, with elevated anxiety symptoms found in populations of alcohol and drug using women (Dixit \& Crum, 2000; Holahan et al., 2003; Schutte et al., 1995). As noted with depressive symptoms, HIV disclosure status was unexpectedly associated with increased anxiety symptoms in the group of pregnant women, indicating that women who had disclosed their HIV status demonstrated elevated anxiety symptom scores on the BAI. Negative consequences of HIV disclosure, such as rejection and isolation, have been reported (Lovejoy, 1990). However, suppressing communication about difficult experiences increases the likelihood of stress-related problems (Greenberg \& Stone, 1992; Holahan et al., 2003; Pennebaker, Colder, \& Sharp, 1990). Therefore, the concept that disclosing one's HIV status would be the first step in communicating about HIV and thereby gaining social support, which in turn could decrease stress and anxiety. The unexpected findings in the current study could be attributed to the shame and stigma that is often associated with HIV, and possibly experienced by this group of pregnant women after they disclosed their HIV status.

The study findings indicated that poor sleep quality was significantly and positively associated with anxiety symptoms in both groups (pregnant and non-pregnant) of women, although conflicting reports are noted in the literature. Published literature reveals sleep disturbances in persons with anxiety symptoms in studies involving both objective (e. g. sleep somnography) assessments (Fuller, Waters, Binks, \& Anderson, 1997; Kripke et al., 2004) and subjective (e. g. self reported) sleep assessments (Kripke et al., 2004; Neubauer, 2004; Nokes \& Kendrew, 2001). Other studies report the absence of significant associations between sleep disturbances and anxiety (Darko, Mitler, \& White, 1995; Norman et al., 1992).

\section{AIM FOUR. PREDICTORS OF DEPRESSIVE SYMPTOMS}

Inherited Vulnerabilities and Depressive Symptoms

Although both inherited and acquired vulnerabilities were explored, acquired vulnerabilities were the only significant predictors of depressive symptoms in this sample of non-pregnant women. Neither family history of mood disorders nor family history of alcohol and/or drug use was a significant predictor of depressive symptoms in the current study. These findings were in contrast to much of the published literature, which reports these inherited vulnerabilities as predictors for depressive symptoms. Family history of mood disorders has been a strong predictor of mood disorders (Gershon et al., 1982; Klein et al., 2001; Weissman et al., 1987; Weissman et al., 1997; Weissman et al., 2005). Many studies of families of adults have shown that children born to parents with major depressive disorder have a three-fold risk of experiencing major depressive disorder themselves (Downey \& Coyne, 1990; Lieb et al., 2002; Williamson et al., 2004). Results of multiple studies have also found a significant positive association between family 
history of substance use and mood disorders (Clark et al., 2004; Kendler, Davis, \& Kessler, 1997; Maier et al., 1994). Differences noted in the current study and previously published literature could be related to the small number of women who reported positive family histories. Only 22 of the 60 non-pregnant women in the sample (36.67\%) reported a family history of alcohol and/or drug use. Thus, the study may have not been sufficiently powered to detect the influence of this variable on depressive symptoms.

\section{Acquired Vulnerabilities and Depressive Symptoms}

The findings indicated that the acquired vulnerabilities of current alcohol and/or drug use and poor sleep quality were predictive of higher depressive symptom scores in this population of non-pregnant African American women. Substance use has been consistently identified in the literature as a predictor of depressive symptoms. The results of the current study are consistent with previous literature (Clark et al., 2004; Holahan et al., 2003; Kendler et al., 1997; Olfson et al., 2000). Alcohol and/or drug use has been associated with depressive symptoms in the general population and many studies have described the relationship between alcohol use and mood disorders (Clark et al., 2004; Dixit \& Crum, 2000; Holahan et al., 2003; Schutte et al., 1995).

Poor sleep quality was a significant predictor of depressive symptoms in this population of non-pregnant African American women. The findings in the current study are consistent with much of the previous literature. Norman and colleagues (Norman et al., 1992) report similar findings in their sample of asymptomatic HIV-infected men and Lee and Miramontes (Lee et al., 2001) report similar findings in their sample of HIVpositive women. The latter study examined the relationship between sleep, depression, and fatigue in a group of HIV-positive women $(N=100)$. While the Lee and Miramontes (Lee et al., 2001) study examined subjective measurements of depressive symptoms and fatigue and objective measurements of sleep efficiency, and the current study examined subjectively reported sleep quality and depressive symptoms, results are comparable.

\section{AIM FIVE. PREDICTORS OF ANXIETY SYMPTOMS}

Inherited Vulnerabilities and Anxiety Symptoms

The findings indicated that acquired vulnerabilities served as predictors of anxiety symptoms in this group of non-pregnant women. Again, the acquired vulnerabilities of current alcohol and/or drug use and poor sleep quality were the only predictors of anxiety symptoms in this group of non-pregnant women. Inherited vulnerabilities, family history of mood disorders and family history of alcohol and/or drug use, were not predictors of anxiety symptoms in this sample. Although important historical markers, the presence of these inherited vulnerabilities were not significant predictors of anxiety symptoms. In addition, family history of mood disorders was present in 22 of the non-pregnant women in the sample (36.67\%), while 22 women (36.67\%) in the group of non-pregnant women 
reported a family history of alcohol and/or drug use. Low numbers of participating pregnant women could have hindered the ability to detect significant associations.

Acquired Vulnerabilities and Anxiety Symptoms

The acquired vulnerabilities that were predictors of depressive and anxiety symptoms in this group of non-pregnant African American women who are HIV-positive were alcohol and/or drug use and sleep quality. The findings indicated that the acquired vulnerability of alcohol and/or drug use significantly predicted anxiety symptoms in this group of non-pregnant African American women. Consistent with previous studies, higher levels of anxiety symptoms have been associated with alcohol and/or drug use in populations of adults (Burke, Burke, \& Rae, 1994; Christie et al., 1988; Kushner et al., 1999) women (K. P. Conway, Compton, Stinson, \& Grant, 2006), African Americans (Ginsburg \& Drake, 2002), and HIV-positive persons (Bing et al., 2001). Alcohol use disorders have been associated with higher rates of major depressive disorders (Goodwin, Kroenke, Hoven, \& Spitzer, 2003).

Although substance use has been well documented as a correlate of mood disorders (R. C. Kessler, McGonagle, Zhao et al., 1994; Regier et al., 1990), the evidence related to sleep assessment, including sleep quality and sleep hygiene is limited. Sleep quality and dysfunction have been studied in HIV-positive women as predictors of fatigue (Mock et al., 2002). Overlapping characteristics may have been present within the current study, as poor sleep quality is a common complaint among patients with HIV infection (Darko et al., 1998; Darko et al., 1995; Mock et al., 2002; Penzak et al., 2000), alcohol use disorders (Dahl et al., 2003), depression (Darko et al., 1992; Mock et al., 2002; Norman et al., 1992), and anxiety (Neubauer, 2004).

Poor sleep quality was noted in the majority of the sample. Although poor sleep quality has been associated with mood disorders (Darko et al., 1992; Mock et al., 2002; Neubauer, 2004; Norman et al., 1992) sleep assessments are not routinely obtained or documented in the medical record (Rubinstein \& Selwyn, 1998). Although the PSQI has been widely used as an instrument for measuring sleep quality, it does not address the physical or environmental characteristics that directly affect sleep quality. The synergistic and circular nature of insomnia and mood disorders has been discussed and bidirectional relationships revealed (Neubauer, 2004). Therefore, comprehensive sleep quality assessments, which include environmental aspects, should be incorporated into routine HIV care.

\section{LIMITATIONS AND STRENGTHS}

This study had four limitations. First, this study implemented a cross-sectional, descriptive correlation design. This research design identifies associations present between and among variables during a specific point in time. Cross-sectional designs do not permit the exploration of changes over time, nor do they permit exploration of cause

and effect. Neither the chronicity of symptoms, nor the recurrences of symptoms are 
captured in cross-sectional studies. Thus, these results concerning levels of anxiety and depressive symptoms, support associations with inherited and acquired vulnerabilities, but do not address causality, changes over time, or disease progression.

The second limitation was convenience sampling; the fact that the entire sample was recruited from health care clinics indicated that these women were receiving health care. Consequently, women who did not receive routine health care were excluded from the sample. This subgroup women, who were not available to participate in this study could have experienced higher or lower rates of depressive and anxiety symptoms than the participants in the current study. Therefore, the results of the current study may be biased, limiting the external validity of the study findings to other populations. Randomly selected study participants would have strengthened the design of the study however this is seldom possible in health-related research.

The majority of the sample in the current study was poor, reporting an annual household income below 5000.00. Higher rates of depressive illness have been reported in poor populations (Everson et al., 2002; Gazmararian et al., 1995; Huurre, Eerola, Rahkonen, \& Aro, 2007). Low levels of education (less than high school degree or equivalent) were noted in only nine $(11.25 \%)$ of the participants. A third of the sample [28 (35\%)] had some college experience, and 19 (23.75\%) reported a Bachelor's degree or higher. Study results show that educated women expose themselves to the risk of HIV infection. The inclusion of a wider range of socioeconomic levels would have enhanced the external validity of the study, although the sample in the current study reflects current HIV demographic trends (Centers for Disease Control and Prevention, 2007c).

Lastly, self-rated and self-reported measures of anxiety and depressive symptoms, as well as alcohol and/or drug use may not accurately capture precise information. Self-reporting of anxiety and depressive symptoms may be limited due to embarrassment and may not address the actual presence of symptoms. The ability of selfreport measurements for depressive and anxiety symptoms to discriminate between persons with true depressive and anxiety symptoms and persons with false positive results is never 100\% accurate (Sharp \& Lipsky, 2002). The CES-D, EPDS, and BAI are all recommended as screening instruments. Self-rated evaluations of depressive and anxiety symptoms have demonstrated both harms and benefits. According to the United States Preventive Services Task Force (United States Preventive Services Task Force, 2002), the potential benefits of screening for depressive disorders include identifying those who need treatment, reducing morbidity and mortality, and improving quality of life, while potential harms of screening include false-positive screening results, adverse effects related to labeling persons with false positive results, as well as treating persons with false-positive results. Since all symptom information was self-reported, a complete accurate account of symptomatology may not have been portrayed, due to factors such as denial or hurried completion of instrumentation.

Conflicting accounts have been published regarding the reporting of psychological symptoms by African Americans. Plant and Sachs-Ericsson (Plant \& Sachs-Ericsson, 2004) and colleagues report that ethnic minority women report more 
depressive symptoms than Caucasian women, while Wallsten (Wallsten, 2000) reports that African Americans also are less likely to report psychological symptoms. George and Lynch (George \& Lynch, 2003) report that African Americans experience greater stress than White Americans, therefore experience greater depressive symptoms than White Americans. However, African Americans are less likely to seek outpatient mental health care (Alegria et al., 2004; K. Wells et al., 2001) than their white counterparts and are underrepresented in published research studies, further limiting comparisons across studies.

Self-reporting of current alcohol and/or drug use was used in the current study. This created another bias; participants may have chosen to not report illegal drug use. Literature illustrates several reasons person may be untruthful regarding alcohol and/or drug use. Fear of being reported to the authorities, fear of legal implications, embarrassment, or distrust of the healthcare system (S. M. Smith et al., 2006). Participants may have engaged in alcohol and/or drug use, but did not want their medical care provider to know, therefore reported no use or lower levels of use. The under reporting of alcohol and/or drug use may have occurred in the pregnant group, as society does not view substance use as acceptable behavior in pregnant women. Despite these factors related to accuracy of self-reported alcohol and /or drug use assessments, literature suggests the majority of self-reports are valid (Alegria et al., 2004; Latkin, 1997; Sobell \& Sobell, 1978). Literature reports the accuracy of self-reported measurements of substance use can be enhanced if the participant is not under the influence of alcohol and/or drugs, completes the assessment or interview in a clinical research setting, and is ensured confidentiality (Sobell \& Sobell, 1978). Although assessments were performed at clinical locations and participants were informed of confidentiality and questionnaire coding, the possibility of inaccurate reports of substance use remains.

Concomitant psychological assessment by a psychologist or psychiatrists would have strengthened the results and possibly given a more accurate portrayal of the mental health of the participants. Additional interviews with the researcher and health care providers could have added to a complete assessment of symptomatology. Follow-up data could have provided additional information regarding results of psychiatric evaluations that were conducted as a result of reported depressive and anxiety symptomatology. Furthermore, the comparison of clinical assessments with questionnaire scores would have provided additional validity to the study. The present study results are reflective of self-reported assessments of depressive and anxiety symptoms in this population of African American women.

Despite these methodological limitations, the present study has several strengths. This was the first study to examine specific inherited and acquired vulnerabilities in African American women who were HIV-positive. Most studies have examined the effects of family history of mood disorders or family history of alcohol and drug use in mentally ill or clinically depressed populations. The present study examined actual predictors of symptoms, which may aid health care providers in the comprehensive care of patients, increasing their ability to anticipate complications based on patients' specific 
family and personal histories. Educational level, income level, alcohol and/or drug use, history of sexual assault or abuse, HIV disclosure status, and sleep quality were acquired vulnerabilities that was explored in the current study. Although educational level, income level, and alcohol and/or drug use are aspects that have previously been identified and studied as predictors of mood disorders, the relationships of these variables as they apply to HIV-positive women have not fully been explored. Women and minorities continue to be underrepresented populations in research studies, particularly research pertaining to HIV/AIDS. As women of color are significantly affected by HIV, and AIDS is the number one killer of African American women between the ages of 25 and 34 in the United States, their inclusion in research becomes important and necessary.

The assessment of history of sexual abuse or assault, HIV disclosure status, and sleep quality were vulnerabilities that have not fully been explored in this population of HIV-positive African American women. Although history of sexual abuse and assault and HIV disclosure status were not predictors of depressive or anxiety symptoms in this population of African American women, these vulnerabilities should be assessed in clinical settings, as they have been shown to be relevant to mood disorders in other studies pertaining to women (Barbe et al., 2004). In this group of HIV-positive women, $33(41.25 \%)$ reported a history of sexual assault. However, 40 women (50\%) reported first sexual experience that occurred when they were younger than age 18 and their partner was at least 3 years older, which legally constitutes statutory rape in the state of Tennessee. Seventeen of these women reported histories of sexual assault; therefore, if the additional 23 women were added to the total number of sexual assault histories, the total would be $53(66.25 \%)$ of the sample.

Additionally, the exclusively African American sample of women in the current study's sample provides an important contribution. Women and minorities continue to be underrepresented in research studies; therefore this study adds information to the body of literature. Although much of the earlier HIV literature reflected White, male, homosexual populations, the demographic characteristics of more recent studies reflect the changing face of AIDS. As HIV continues to disproportionately affect minorities in the United States, additional studies comprised of African American samples becomes imperative.

An additional strength of the study was that subjects were recruited from outpatient clinics that employed specialists in the field of HIV. The reputation of the clinics for their expertise in HIV care and management resulted in population of patients that encompassed a variety of socio-economic levels. Although most of the sample that participated in the current study was poor and unemployed, the expertise of the clinical sites increased the likelihood that patients from a wider range of socioeconomic levels was available in the population from which the sample was recruited. Wider ranges of socioeconomic levels and inclusion of both rural and urban populations would have enhanced the external validity of the study. 


\section{IMPLICATIONS}

The results of this study have important implications for health care practice. Increased awareness of potential vulnerabilities to depressive and anxiety symptoms by healthcare providers may lead to increased screening for mood disorders. Research has shown that primary care physicians fail to recognize and treat $30 \%$ to $50 \%$ of adult depressed patients (Simon, Goldberg, Von Korff, \& Ustun, 2002; Von Korff \& Simon, 1996). Moreover, failure to detect depression may be greater for African American or Hispanic patients and for patients under 35 years (Borowsky et al., 2000). Therefore, the need for accurate, comprehensive screening and assessment of depressive and anxiety symptoms in the health care setting remains crucial. Comprehensive screening as a holistic approach may assist in the integration of mental health services into primary care (Dodds et al., 2000).

The current study revealed the presence of sub-clinical levels of anxiety and depressive symptoms in some participants and potentially clinical levels in others, establishing the need for individual, ongoing assessment of vulnerabilities. Identification of patients needing further evaluation and treatment may decrease adverse events and psychological related hospitalizations. About 50\% of women in the sample had scores requiring referral to the social worker. Although no participant in the current study required immediate hospitalization, two of the participants were started on antidepressants the day of study participation. These two women were referred to their provider based on their elevated study questionnaire scores and additional clinical assessment prior to initiating treatment antidepressant drugs. Current treatment with antidepressants was not an exclusion criterion for the current study. Nine $(15 \%)$ of the non-pregnant women $(n=60)$ were on antidepressants at the time of their participation in the study; nevertheless eight of these women had elevated depressive symptom scores. Therefore, the assessment of adequacy and efficacy of psychiatric medications is indicated as an ongoing aspect of comprehensive health care. In this sample of women, medication treatment was not consistently associated with sub-clinical symptom levels. No assessment was made, however regarding the duration of antidepressant therapy.

The overwhelming presence of poor sleep quality in the sample, combined with the association of poor sleep quality with anxiety and depressive symptoms, demonstrates the need for routine assessment and documentation of sleep practices. Although sleep disturbances were common in the HIV-positive women in this study and other studies, assessment of sleep is not routinely documented in patients' medical records (Rubinstein $\&$ Selwyn, 1998). The adverse effects of poor sleep quality are multifaceted. Sleep and health are impacted by the immune system. Sleep disturbances have been shown to worsen as the course of HIV worsens (Darko et al., 1998; Mock et al., 2002; Nokes \& Kendrew, 2001; Sobell \& Sobell, 1978). Poor sleep quality has been associated with increased morbidity and mortality in HIV-positive persons (Darko et al., 1998). Immunological molecules, including cytokines, impact sleep regulation (Motivala, Sarfatti, Olmos, \& Irwin, 2005). Impaired sleep is often a symptom of psychiatric disorders, including anxiety and depression (Neubauer, 2004), while chronic insomnia is a risk factor for the development and recurrence of psychiatric disorders (Lustberg \& Reynolds, 2000). Women with poor sleep quality were not being treated for insomnia, 
indicating either a lack of appropriate screening or insufficient communication with their provider. Therefore, assessment of sleep quality and sleep disturbances should be part of comprehensive medical care, and documented as such particularly in patients who are HIV positive.

Increased adherence to antiretroviral medications may prolong the duration of the regimen as well as decrease transmission of medication resistant virus (A. Ammassari et al., 2004). The relationship of decreased antiretroviral adherence and mood disorders has been well established. Depression in persons who are HIV-positive often results in decreased adherence to antiretroviral medications (Chander, Lau, \& Moore, 2006; Starace et al., 2002) and increased risk-taking behaviors (Croughan, Miller, Wagelin, \& Whitman, 1982; Hutton, Lyketsos, Zenilman, Thompson, \& Erbelding, 2004; Valente, 2003). A significant percentage of the participants in this study had CES-D, EPDS, and BAI scores requiring immediate referral to the social worker, suggesting that patients were not routinely assessed for signs and symptoms of depression or anxiety, despite the fact that they were routinely receiving health care. As adherence to HAART can be reduced by comorbid psychiatric disorders (Starace et al., 2002) it is imperative that comprehensive psychosocial assessments, including routine screening for depressive and anxiety symptoms, be a routine aspect of HIV care, similar to the practice of obtaining laboratory evaluations.

Adherence to antiretroviral medications has been inversely related to substance use in HIV-positive persons (Chander et al., 2006; Welch, 2000). The most common substance used by women in the current study was alcohol, with 19 of the 24 women that reported substance use reporting recent alcohol use. Routine assessment of substance use is crucial to comprehensive HIV care. Collectively, these study results indicate the importance of comprehensive assessments of potential vulnerabilities. Inquiry regarding substance use and sleep quality, along with interval antiretroviral adherence assessments, may increase the early identification of adherence problems. Noncompliance is one of the main causes of antiretroviral resistance. Identification of barriers to adherence may lengthen the time the person is able to use a specific antiretroviral regimen. In addition to comprehensive assessment, thorough documentation is vital to patient care.

The vast majority (92.5\%) of the sample had disclosed their HIV status to others. Thus, it was not possible to show an association between disclosure and depressive and anxiety symptoms as there was limited variability in HIV disclosure status. Despite this finding, disclosure remains an important aspect of HIV. Increased disclosure of HIV status to sex partners has the potential to decrease HIV transmission. Failure to disclose HIV status can have devastating effects on public health. The most effective means of HIV prevention among sexually active persons is consistent condom use (Centers for Disease Control and Prevention, 2005). Lack of consistent condom use has been shown to be prevalent in persons that did not disclose their HIV status to their partner (Chander et al., 2006; Niccolai et al., 1999; Stein et al., 1998; Stein \& Samet, 1999). Disclosure of HIV status to family members and/or friends may help decrease stigma related to HIV, while increasing support systems for patients. Stigma has been shown to "thrive in an environment of ignorance and half-truths" (Valdiserri, 2002) p. 342). Therefore, HIV 
education and awareness would be the first step utilized to decrease stigma. Although HIV disclosure was not a predictor of anxiety or depressive symptoms in the present study because virtually all participants reported disclosing their status, the assessment of HIV disclosure remains an important aspect of HIV care, and should be addressed regularly as part of an ongoing psychosocial assessment.

Although cross-sectional data do not describe incidences that are present over time, they do describe a snapshot assessment of individual characteristics. In the present study, the participants expressed a multitude of psychological and social burdens that would not have been assessed in a regular clinical appointment. The completion of the study instruments led many patients to expound upon their experiences, thereby opening communication with the clinical researcher. This type of revelation was often accompanied by tears and expressions of relief that they were able to share their experiences, concerns, and secrets with someone. Some participants reported being victims of incest; while others disclosed a spouse's suicide after a participant was diagnosed with HIV; another reported weekly childhood rape by the man that transported the participant to church every Sunday; and another reported displacement from her New Orleans home and trauma related to hurricane Katrina. These unplanned discoveries suggest that including psychosocial assessments in the care of persons who are HIV positive would add additional support for the patient, as well as enhance providers' insights into the lives of patients. Ongoing assessments, such as social work or case management assessments at every visit, not only when a patient expresses a concern or crisis, could provide an opportunity for patients to share some of these challenging aspects of their lives. The inability to share traumatizing experiences may increase depressive symptoms in this population. Depressive symptoms have been shown to negatively affect HIV/AIDS treatment outcomes (A. Ammassari et al., 2004; Ickovics et al., 2001; Lyketsos et al., 1996; Starace et al., 2002; Vyavaharkar et al., 2007), often impacting adherence with HAART (A. Ammassari et al., 2004; A. A. M. Ammassari et al., 2003; Starace et al., 2002; Vyavaharkar et al., 2007).

\section{THEORETICAL IMPLICATIONS}

Study findings provide partial support for the stress diathesis model as two of the acquired vulnerabilities were significant predictors of anxiety and depressive symptoms. The identification of vulnerabilities, both inherited and acquired, is an important tool in comprehensive psychosocial assessments. The occurrence of stressful life events has been found to increase the probability of a depressive episode in elderly populations (Emmerson, Burvill, Finlay-Jones, \& Hall, 1989), as well as populations of HIV-positive women (Moore et al., 1999). Two acquired vulnerabilities, alcohol and/or drug use and poor sleep quality, were significant predictors of anxiety and depressive symptoms. Comorbid alcohol and/or drug use and psychiatric disorders have been well-documented in the general population (Helzer \& Pryzbeck, 1988; R. C. Kessler et al., 1997; Regier et al., 1990; Starace et al., 2002; Vyavaharkar et al., 2007). The relationship between sleep quality and depression in the HIV-positive population is also well documented (Darko et al., 1992; Mock et al., 2002; Norman et al., 1992). The inherited vulnerability of family history of mood disorders was associated with higher CES-D, EPDS, and BAI scores in 
the study sample. Findings were similar to previously published literature (Gutman \& Nemeroff, 2003) suggesting that the assessment of pertinent inherited and acquired vulnerabilities should be a part of the routine assessment of African American women who are HIV-positive to identify women that may be at an increased risk for mood disorders.

\section{FUTURE RESEARCH}

Study findings provide guidance for future research in this population of women. Future directions might include a longitudinal study that would follow participants over time, thereby assessing the presence of vulnerabilities, depressive and anxiety symptoms and other long-term outcomes related to the HIV-diagnosis. This type of research would add to the understanding of the effects of stress over time, which may be especially important in immunocompromised populations, such as those persons with HIV. Poor sleep quality was a predictor of anxiety and depressive symptoms in the current study and insomnia has been associated with psychiatric disorders, including depression (Lustberg \& Reynolds, 2000). Future studies should include assessment of sleep quality and sleep hygiene, as well as test of established interventions that could improve sleep quality in the study population.

Conflicting evidence regarding the impact of sleep quality and HIV disease progression is noted in the literature. The impact of sleep quality on immunity (decrease in CD4 count) has been illustrated in several studies (Darko et al., 1992; Mock et al., 2002; Norman et al., 1992). Confounding results were noted in other studies involving HIV-infected populations (Nokes, Chidekel, \& Kendrew, 1999; Perkins et al., 1994; Rubinstein \& Selwyn, 1998), showing no effect of insomnia on immunologic markers (CD4 count). The relationship between sleep and CD4 counts was not assessed in the current study, although on average participants had counts of 505. The effect of poor sleep quality in HIV-positive women has been well documented (Lee et al., 2001; Mock et al., 2002). Sleep quality has the ability to affect many facets of well-being. Quality of life in HIV-positive women has been associated with increased sleep quality and other health-promoting behaviors such as exercise, stress management, and healthy eating habits (Gielen, McDonnell, Wu, O'Campo, \& Faden, 2001). Therefore, future studies should include not only sleep assessments, but also interventions that can improve sleep quality.

Stigma, shame, and fear continue to be additional stressors related to HIV, which often delay or prevent treatment (Valdiserri, 2002). In the current study, six of the participants (7.5\%) had not disclosed their HIV-positive status. The CDC (Branson et al., 2006) estimates that of the approximately 1 to 1.2 million persons living with HIV in the United States about 25\% (252,000-312,000 persons) were unaware of their infection. It is thought that many of these persons have unknowingly transmitted HIV. The lack of awareness of current HIV infection leads to two main issues: 1) increased numbers of HIV transmission and 2) lack of medical care, thereby increasing morbidity and mortality related to HIV/AIDS (Branson et al., 2006). Therefore, future research should concentrate on the factors that are related to these difficulties. 
Future research studies should include structured clinical interviews as well as self-report measurements of depressive and anxiety symptoms. This comprehensive approach would provide a more thorough and accurate mental health assessment, as selfreport measures are often confounded by denial and under reporting of symptoms. This comprehensive approach would identify persons in need of additional screening, referral, and treatment. Self-esteem measurement might provide insights regarding the mental health status of African American women who are HIV-positive. Research has shown that self esteem decreases as a result of severe mental illness (Shahar \& Davidson, 2003). Additionally, poor self-esteem commonly occurs in the context of depressive symptoms, particularly in women.

Future research should also include a comprehensive housing assessment, as housing situations in this population are often unstable, unsafe, and nonpermanent. Almost half (45\%) of the HIV-positive persons served by the Ryan White Care Act in $2002(N=844,687)$ reported non-permanent housing (USDHHS Health Resources and Services Administration, 2002). Although housing is transient, and often temporary for persons with HIV, this issue was not systematically explored in the study sample. However, several study participants $(9,11.25 \%)$ shared concerns related to this issue. Concerns such as these, if persistent, likely serve as an acquired vulnerability that contributes to the risk for depression and anxiety.

Early postnatal screening measures should be studied further, especially in minority populations, as they have been grossly underrepresented in previous research. Literature suggests that EPDS scores within the first few days of delivery are highly correlated with EPDS obtained at four to six weeks postpartum (Chabrol \& Teissedre, 2004; Hannah et al., 1992). Implementing routine screening of postpartum women before hospital discharge may identify those women at risk for postpartum depression earlier, thereby decreasing risks associated with postpartum depression for both mother and baby.

Finally, additional research should include qualitative or mixed methods studies to explore the specific factors that contribute to mood disorders, HIV disclosure, and decision making processes related to family planning in the HIV-positive population. Qualitative research can aid the progress of nursing research by guiding research that has a direct impact on nursing practice (Barroso \& Powell-Cope, 2000). There are existing qualitative research studies in the HIV literature regarding adherence to antiretroviral medication and barriers to care (Bunting et al., 1999; Bunting \& Seaton, 1999), and a qualitative meta-analysis, or metasynthesis, explored the experience of living with HIV (Barroso \& Powell-Cope, 2000). Future research could use focus groups in different types of populations to explore sleep quality and sleep hygiene practices, as poor sleep quality had an impact on depressive and anxiety symptoms in this study. Sleep is affected by many factors which should be explored in other populations as well. Environmental factors such as temperature, noise levels, numbers of children in the home, numbers of people in the bed, and insect infestation in the home were some of the points the participants reported at the end of questionnaire completion. Findings from the current study reveal that women present with a multitude of histories and vulnerabilities that are 
often complex. Qualitative research may lead to a greater understanding of the meaning of being HIV-positive, which may help researchers and healthcare providers gain understanding, rather than relying on preconceived ideas of what the experience of living with HIV is truly like.

\section{CONCLUSIONS}

This study provided insights regarding the impact of vulnerabilities, both inherited and acquired, on depressive and anxiety symptoms. Study findings emphasize the importance of comprehensive assessments and ongoing communication with patients. Participants were willing to disclose additional challenges they faced on a daily basis and often valued the opportunity to share their personal stories. Disclosing their concerns, for many participant, was therapeutic. This study highlighted alcohol and/or drug use and poor sleep quality as predictors of depressive and anxiety symptoms in a sample of non-

pregnant African American women who were HIV-positive. Key findings in both groups of women included significant associations between both inherited and acquired vulnerabilities and elevated depressive and anxiety symptom scores. Alcohol and/or drug use and poor sleep quality were the acquired vulnerabilities that were significantly and positively associated with increased depressive and anxiety scores in both groups of women. Family history of mood disorders was significantly and positively correlated with depressive and anxiety symptoms in the group of pregnant women; while family history of mood disorders was significantly and positively associated with elevated anxiety symptom scores in the group of non-pregnant women. Additional findings in the non-pregnant group revealed an unexpected significant positive association between HIV disclosure status and elevated CES-D scores, indicating that women that had disclosed their HIV status reported more depressive symptoms. However, this association did not occur for EPDS scores.

Developing specific evidence-based interventions remains an important aspect of clinical research and practice, particularly with regard to the poor sleep quality that occurred in an important proportion of study participants. While the participants in this study were exclusively African American women who were HIV-positive, findings could be relevant for other populations who share similar experiences and vulnerabilities. Underserved populations, including racial and ethnic minorities, continue to receive inadequate mental health treatment, often because of stigma and poor access to care. Decreasing the stigma related to both HIV and mental illness, may increase access to healthcare services and improve health outcomes in this population. Addressing unmet needs of traditionally underserved populations in the United States is a social imperative for all Americans as the experiences and unmet needs of these populations have important implications for the future health of this nation. 


\section{LIST OF REFERENCES}

Adebimpe, V. R. (1981). Overview: white norms and psychiatric diagnosis of black patients. American Journal of Psychiatry, 138(3), 279-285.

Alegria, M., Canino, G., Rios, R., \& et al. (2004). Inequalities in Use of Specialty Mental Health Services Among Latinos, African Americans, and Non-Latino Whites. Year Book of Psychiatry \& Applied Mental Health, 184-185.

American Psychiatric Association Task Force. (2000). Handbook of psychiatric measures. Washington, DC: American Psychiatric Association.

Ammassari, A., Antinori, A., Aloisi, M. S., Trotta, M. P., Murri, R., Bartoli, L., et al. (2004). Depressive symptoms, neurocognitive impairment, and adherence to highly active antiretroviral therapy among HIV-infected persons. Psychosomatics, 45(5), 394-402.

Ammassari, A. A. M., Starace, F. M., Aloisi, M. S. M., Trotta, M. P. M., Murri, R. M., d'Arminio Monforte, A. M., et al. (2003). Medication adherence among HIV+ adults: Effects of cognitive dysfunction and regimen complexity. Neurology, 61(5), 723-724.

Anderson, G. D. (2002). Sex differences in drug metabolism: cytochrome P-450 and uridine diphosphate glucuronosyltransferase. Journal of Gender-Specific Medicine, 5(1), 25-33.

Atkinson, J. J., Grant, I., Kennedy, C. J., Richman, D. D., Spector, S. A., \& McCutchan, J. (1988). Prevalence of psychiatric disorders among men infected with human immunodeficiency virus: A controlled study. Arch Gen Psychiatry, 45, 859-964.

Ayanian, J. Z. (1994). Race, class, and the quality of medical care. JAMA, 271(15), 12071208.

Barbe, R. P., Bridge, J. A., Birmaher, B., Kolko, D. J., \& Brent, D. A. (2004). Lifetime history of sexual abuse, clinical presentation, and outcome in a clinical trial for adolescent depression. Journal of Clinical Psychiatry, 65(1), 77-83.

Barker-Collo, S., \& Read, J. (2003). Models of response to childhood sexual abuse: their implications for treatment. Trauma Violence \& Abuse, 4(2), 95-111.

Barnard, M., \& McKeganey, N. (2004). The impact of parental problem drug use on children: what is the problem and what can be done to help? Addiction, 99(5), 552-559.

Barroso, J. (2002). Living with illness. HIV-related fatigue: nursing interventions to help patients manage. American Journal of Nursing, 102(5), 83-86.

Barroso, J., Carlson, J. R., \& Meynell, J. (2003). Physiological and psychosocial markers associated with HIV-related fatigue. . Clinical Nursing Research, 12(1), 49-68.

Barroso, J., Pence, B. W., Salahuddin, N., Harmon, J. L., \& Leserman, J. (2008). Physiological Correlates of HIV-related fatigue. Clinical Nursing Research, 17(1), 5-19.

Barroso, J., \& Powell-Cope, G. M. (2000). Metasynthesis of qualitative research on living with HIV infection. Qualitative Health Research, 10(3), 340-353.

Bauer, L. O., Costa, L., \& Hesselbrock, V. M. (2001). Effects of alcoholism, anxiety and depression on P300 in women: a pilot study. Journal of Studies on Alcohol, 62(5), 571-579. 
Beardslee, W. R., Versage, E. M., \& Gladstone, T. R. (1998). Children of affectively ill parents: a review of the past 10 years. Journal of the American Academy of Child \& Adolescent Psychiatry, 37(11), 1134-1141.

Beatty, L. A., Wheeler, D., \& Gaiter, J. (2004). HIV prevention research for African Americans: current and future directions. Journal of Black Psychology, 30, 40-58.

Beck, A. T., Epstein, N., Brown, G., \& Steer, R. A. (1988). An inventory for measuring clinical anxiety: psychometric properties. Journal of Consulting \& Clinical Psychology, 56(6), 893-897.

Beck, A. T., Rial, W. Y., \& Rickels, K. (1974). Short form of depression inventory: cross-validation. Psychological Reports, 34(3), 1184-1186.

Beck, C. T. (1996). A meta-analysis of predictors of postpartum depression. Nursing Research, 45(5), 297-303.

Beck, C. T. (1998). A checklist to identify women at risk for developing postpartum depression. JOGNN: Journal of Obstetric, Gynecologic, \& Neonatal Nursing, 27(1), 39-46.

Beck, C. T. (2001). Predictors of postpartum depression: an update. Nursing Research, 50(5), 275-285.

Beck, C. T., \& Gable, R. K. (2000). Postpartum Depression Screening Scale: development and psychometric testing. Nursing Research, 49(5), 272-282.

Berkman, L. F., Berkman, C. S., Kasl, S., Freeman, D. H., Jr., Leo, L., Ostfeld, A. M., et al. (1986). Depressive symptoms in relation to physical health and functioning in the elderly. American Journal of Epidemiology, 124(3), 372-388.

Bing, E. G., Burnam, M. A., Longshore, D., Fleishman, J. A., Sherbourne, C. D., London, A. S., et al. (2001). Psychiatric disorders and drug use among human immunodeficiency virus-infected adults in the United States. Archives of General Psychiatry, 58(8), 721-728.

Blazer, D. G., Kessler, R. C., McGonagle, K. A., \& Swartz, M. S. (1994). The prevalence and distribution of major depression in a national community sample: The national comorbidity study. . American Journal of Psychiatry, 151, 979-986.

Blehar, M. D., \& Oren, D. A. (1995). Women's increased vulnerability to mood disorders: Integrating psychobiology and epidemiology. Depression, 3, 3-12.

Boggs, W. (2007). Disclosure of HIV status and sexual orientation may influence CD4 cell counts. Psychsomatic Medicine, 69, 74-80.

Bolden, L., \& Wicks, M. N. (2005). Length of stay, admission types, psychiatric diagnoses, and the implications of stigma in African Americans in the nationwide inpatient sample. Issues in Mental Health Nursing, 26(10), 1043-1059.

Borowsky, S. J., Rubenstein, L. V., Meredith, L. S., Camp, P., Jackson-Triche, M., \& Wells, K. B. (2000). Who is at risk of nondetection of mental health problems in primary care? . Journal of General Internal Medicine, 15, 381-388.

Boyd-Franklin, N. (1987). Group therapy for black women: A therapeutic support model. . American Journal of Orthopsychiatry, 57(3), 394-401.

Branson, B. M., Handsfield, H. H., Lampe, M. A., Janssen, R. S., Taylor, A. W., Lyss, S. B., et al. (2006). Revised recommendations for HIV testing of adults, adolescents, and pregnant women in health-care settings.[see comment]. Morbidity \& Mortality Weekly Report Recommendations \& Reports, 55(RR-14), 1-17; quiz CE11-14. 
Breslau, N., Schultz, L., \& Peterson, E. (1995). Sex differences in depression: A role for preexisting anxiety. Psychiatry Research, 58(1), 1-12.

Brown, T. A., Campbell, L. A., Lehman, C. L., Grisham, J. R., \& Mancill, R. B. (2001). Current and lifetime comorbidity of the DSM-IV anxiety and mood disorders in a large clinical sample. Journal of Abnormal Psychology, 110(4), 585-599.

Bunting, S. M., Bevier, D. J., \& Baker, S. K. (1999). Poor women living with HIV: selfidentified needs. Journal of Community Health Nursing, 16(1), 41-52.

Bunting, S. M., \& Seaton, R. (1999). Health care participation of perinatal women with HIV: what helps and what gets in the way? Health Care for Women International, 20(6), 563-578.

Burke, J. D., Jr., Burke, K. C., \& Rae, D. S. (1994). Increased rates of drug abuse and dependence after onset of mood or anxiety disorders in adolescence. Hospital \& Community Psychiatry, 45(5), 451-455.

Buysse, D. J., Reynolds, C. F., 3rd, Monk, T. H., Berman, S. R., \& Kupfer, D. J. (1989). The Pittsburgh Sleep Quality Index: a new instrument for psychiatric practice and research. Psychiatry Research, 28(2), 193-213.

Carpenter, J. S., \& Andrykowski, M. A. (1998). Psychometric evaluation of the Pittsburgh Sleep Quality Index. Journal of Psychosomatic Research, 45(1 Spec No), 5-13.

Casswell, S., Pledger, M., \& Hooper, R. (2003). Socioeconomic status and drinking patterns in young adults.[see comment]. Addiction, 98(5), 601-610.

Cavanaugh, S., Clark, D. C., \& Gibbons, R. D. (1983). Diagnosing depression in the hospitalized medically ill. Psychosomatics, 24(9), 809-815.

CDC. (2006). WISQARS Leading causes of death reports, 1999-2004. Retrieved March 1, 2007., from http://webappa.cdc.gov/sasweb/ncipc/leadcaus10.html.

Centers for Control and Prevention. (2002). U.S. Public Health Service Task Force Recommendations for Use--of Antiretroviral Drugs in Pregnant HIV-1--Infected Women for Maternal Health and Interventions To Reduce Perinatal HIV-1 Transmission in the United States Retrieved 4-7-08, from http://www.cdc.gov/MMWR/preview/mmwrhtml/rr5118a1.ht

Centers for Control and Prevention. (2007, June, 2007). Cases of HIV infection and AIDS in the United States, and dependent areas, 2005. HIV/AIDS Surveillance Report, Volume 17, Revised Edition, June 2007 Retrieved April 15, 2008, from http://www.cdc.gov/hiv/topics/surveillance/resources/reports/2005report/default.h tm

Centers for Control and Prevention. (2008, March, 2008). HIV/AIDS in the United States. HIV/AIDS Fact Sheet: Populations/Surveillance Retrieved 4-21-08, from http://www.cdc.gov/hiv/resources/factsheets/us.htm

Centers for Disease Control and Prevention. (2000). HIV-related knowledge and stigma-United States, 2000. MMWR - Morbidity \& Mortality Weekly Report, 49(47), 1062-1064.

Centers for Disease Control and Prevention. (2002). HIV testing among pregnant women--United States and Canada, 1998-2001. MMWR - Morbidity \& Mortality Weekly Report, 51(45), 1013-1016. 
Centers for Disease Control and Prevention. (2003). Increases in HIV diagnoses--29 States, 1999-2002. MMWR - Morbidity \& Mortality Weekly Report, 52(47), 1145 1148.

Centers for Disease Control and Prevention. (2004). Diagnoses of HIV/AIDS--32 States, 2000-2003. MMWR - Morbidity \& Mortality Weekly Report, 53(47), 1106-1110.

Centers for Disease Control and Prevention. (2005). HIV transmission among black women--North Carolina, 2004. MMWR - Morbidity \& Mortality Weekly Report, 54(4), 89-94.

Centers for Disease Control and Prevention. (2005) HIV/AIDS Surveillance. Retrieved April 24, 2005.Available at http://www.cdc.gov.hiv/graphics.htm.).

Centers for Disease Control and Prevention. (2007a, June 2007). HIV/AIDS Among Women, Fact sheet. Retrieved August 6, 2007, 2007, from http://www.cdc.gov/hiv/topics/women/resources/factsheets/women.htm.

Centers for Disease Control and Prevention. (2007b). HIV/AIDS diagnoses among blacks--Florida, 1999-2004. MMWR - Morbidity \& Mortality Weekly Report, 56(4), 69-73.

Centers for Disease Control and Prevention. (2007c). Racial/ethnic disparities in diagnoses of HIV/AIDS--33 states, 2001-2005. MMWR - Morbidity \& Mortality Weekly Report, 56(9), 189-193.

Chabrol, H., \& Teissedre, F. (2004). Relation between Edinburgh Postnatal Depression Scale scores at 2-3 days and 4-6 weeks postpartum. Journal of Reproductive \& Infant Psychology, 22(1), 33-39.

Chander, G., Lau, B., \& Moore, R. D. (2006). Hazardous alcohol use: a risk factor for non-adherence and lack of suppression in HIV infection. Journal of Acquired Immune Deficiency Syndromes: JAIDS, 43(4), 411-417.

Christie, K. A., Burke, J. D., Jr., Regier, D. A., Rae, D. S., Boyd, J. H., \& Locke, B. Z. (1988). Epidemiologic evidence for early onset of mental disorders and higher risk of drug abuse in young adults. American Journal of Psychiatry, 145(8), 971975.

Ciesla, J. A., \& Roberts, J. E. (2001). Meta-analysis of the relationship between HIV infection and risk for depressive disorders. American Journal of Psychiatry, 158(5), 725-730.

Clark, D. B., Cornelius, J., Wood, D. S., \& Vanyukov, M. (2004). Psychopathology risk transmission in children of parents with substance use disorders. American Journal of Psychiatry, 161(4), 685-691.

Cochran, S. D., \& Mays, V. M. (1994). Depressive distress among homosexually active African American men and women. American Journal of Psychiatry, 151(4), 524529.

Cohan, D. (2003). Perinatal HIV: special considerations. Topics in HIV Medicine, 11(6), 200-213.

Cohen, J. (1987). Statistical power analysis for the behavioral sciences (Rev ed). Hillsdale: Lawrence Erlbaum Assoc.

Conway, K. P., Compton, W., Stinson, F. S., \& Grant, B. F. (2006). Lifetime comorbidity of DSM-IV mood and anxiety disorders and specific drug use disorders: results from the National Epidemiologic Survey on Alcohol and Related Conditions. Journal of Clinical Psychiatry, 67(2), 247-257. 
Conway, M., Mendelson, M., Giannopoulos, C., Csank, P. A., \& Holm, S. L. (2004). Childhood and adult sexual abuse, rumination on sadness, and dysphoria. Child Abuse \& Neglect, 28(4), 393-410.

Cooper, P., \& Murray, L. (1997). Prediction, detection, and treatment of postnatal depression. Archives of Disease in Childhood, 77(2), 97-99.

Cooper, P. J., \& Murray, L. (1998). Postnatal depression.[see comment]. BMJ, 316(7148), 1884-1886.

Cox, J. L., Chapman, G., Murray, D., \& Jones, P. (1996). Validation of the Edinburgh Postnatal Depression Scale (EPDS) in non-postnatal women. Journal of Affective Disorders, 39(3), 185-189.

Cox, J. L., Holden, J. M., \& Sagovsky, R. (1987). Detection of postnatal depression. Development of the 10-item Edinburgh Postnatal Depression Scale. British Journal of Psychiatry, 150, 782-786.

Croughan, J. L., Miller, J. P., Wagelin, D., \& Whitman, B. Y. (1982). Psychiatric illness in male and female narcotic addicts. Journal of Clinical Psychiatry, 43(6), 225228.

Cruess, D. G., Douglas, S. D., Petitto, J. M., Have, T. T., Gettes, D., Dube, B., et al. (2005). Association of resolution of major depression with increased natural killer cell activity among HIV-seropositive women. American Journal of Psychiatry, 162(11), 2125-2130.

Cruess, D. G., Petitto, J. M., Leserman, J., Douglas, S. D., Gettes, D. R., Ten Have, T. R., et al. (2003). Depression and HIV infection: impact on immune function and disease progression. Cns Spectrums, 8(1), 52-58.

Currie, S. R., Wilson, K. G., \& Curran, D. (2002). Clinical significance and predictors of treatment response to cognitive-behavior therapy for insomnia secondary to chronic pain. Journal of Behavioral Medicine, 25(2), 135-153.

Dahl, R. E., Williamson, D. E., Bertocci, M. A., Stolz, M. V., Ryan, N. D., Ehlers, C. L., et al. (2003). Spectral analyses of sleep EEG in depressed offspring of fathers with or without a positive history of alcohol abuse or dependence: a pilot study. Alcohol, 30(3), 193-200.

Daly, M. C., Duncan, G. J., McDonough, P., \& Williams, D. R. (2002). Optimal indicators of socioeconomic status for health research. American Journal of Public Health, 92(7), 1151-1157.

Darko, D. F., McCutchan, J. A., Kripke, D. F., Gillin, J. C., \& Golshan, S. (1992). Fatigue, sleep disturbance, disability, and indices of progression of HIV infection. American Journal of Psychiatry, 149(4), 514-520.

Darko, D. F., Mitler, M. M., \& Miller, J. C. (1998). Growth hormone, fatigue, poor sleep, and disability in HIV infection. Neuroendocrinology, 67(5), 317-324.

Darko, D. F., Mitler, M. M., \& White, J. L. (1995). Sleep disturbance in early HIV infection. Focus: a Guide to AIDS Research, 10(11), 5-6.

Dennis, C. L. (2004). Can we identify mothers at risk for postpartum depression in the immediate postpartum period using the Edinburgh Postnatal Depression Scale? Journal of Affective Disorders, 78(2), 163-169.

Dennis, C. L., Janssen, P. A., \& Singer, J. (2004). Identifying women at-risk for postpartum depression in the immediate postpartum period. Acta Psychiatrica Scandinavica, 110(5), 338-346. 
Dickinson, L. M., deGruy, F. V., 3rd, Dickinson, W. P., \& Candib, L. M. (1998). Complex posttraumatic stress disorder: evidence from the primary care setting. General Hospital Psychiatry, 20(4), 214-224.

Dixit, A. R., \& Crum, R. M. (2000). Prospective study of depression and the risk of heavy alcohol use in women. American Journal of Psychiatry, 157(5), 751-758.

Dodds, S., Blaney, N. T., Nuehring, E. M., Blakley, T., Lizzotte, J. M., Potter, J. E., et al. (2000). Integrating mental health services into primary care for HIV-infected pregnant and non-pregnant women: Whole Life--a theoretically derived model for clinical care and outcomes assessment. General Hospital Psychiatry, 22(4), 251260.

Dohrenwend, B. P., \& Dohrenwend, B. S. (1969). Social status and psychological disorder: A causal inquiry. New York: Wiley.

Dohrenwend, B. P., \& Dohrenwend, B. S. (1979). The conceptualization and measurement of stressful life events: An overview of the issues. The psychobiology of the depressive disorders: Implications for the effects of stress. San Diego: Academic Press.

Dohrenwend, B. P., Levav, I., Shrout, P. E., Schwartz, S., Naveh, G., Link, B. G., et al. (1992). Socioeconomic status and psychiatric disorders: the causation-selection issue. Science, 255(5047), 946-952.

Downey, G., \& Coyne, J. C. (1990). Children of depressed parents: An integrative review. Psychology Bulletin, 108, 50-76.

Emmerson, J. P., Burvill, P. W., Finlay-Jones, R., \& Hall, W. (1989). Life events, life difficulties and confiding relationships in the depressed elderly. British Journal of Psychiatry, 155, 787-792.

Evans, D. L., Staab, J., Ward, H., Leserman, J., Perkins, D. O., Golden, R. N., et al. (1996). Depression in the medically ill: management considerations. Depression \& Anxiety, 4(4), 199-208.

Evans, D. L., Ten Have, T. R., Douglas, S. D., Gettes, D. R., Morrison, M., Chiappini, M. S., et al. (2002). Association of depression with viral load, CD8 T lymphocytes, and natural killer cells in women with HIV infection. American Journal of Psychiatry, 159(10), 1752-1759.

Everson, S. A., Maty, S. C., Lynch, J. W., Kaplan, G. A., Everson, S. A., Maty, S. C., et al. (2002). Epidemiologic evidence for the relation between socioeconomic status and depression, obesity, and diabetes. Journal of Psychosomatic Research, 53(4), 891-895.

Evins, G. G., Theofrastous, J. P., \& Galvin, S. L. (2000). Postpartum depression: a comparison of screening and routine clinical evaluation. American Journal of Obstetrics \& Gynecology, 182(5), 1080-1082.

Ferrando, S., Evans, S., Goggin, K., Sewell, M., Fishman, B., \& Rabkin, J. (1998). Fatigue in HIV illness: relationship to depression, physical limitations, and disability. Psychosomatic Medicine, 60(6), 759-764.

Fiore, T., Flanigan, T., Hogan, J., Cram, R., Schuman, P., Schoenbaum, E., et al. (2001). HIV infection in families of HIV-positive and 'at-risk' HIV-negative women. AIDS Care, 13(2), 209-214. 
Fogel, C. I., \& Martin, S. L. (1992). The mental health of incarcerated women... including commentary by Anderson NLR, Murphy SA, and Dickson LAS with author response. Western Journal of Nursing Research, 14(1), 30-47.

Fox, J. (1997). Applied regression analysis, linear models, and related methods. Oaks, CA: Sage Publications.

Fuller, K. H., Waters, W. F., Binks, P. G., \& Anderson, T. (1997). Generalized anxiety and sleep architecture: a polysomnographic investigation. Sleep, 20(5), 370-376.

Gazmararian, J. A., James, S. A., \& Lepkowski, J. M. (1995). Depression in black and white women. The role of marriage and socioeconomic status. Annals of Epidemiology, 5(6), 455-463.

George, L. K., \& Lynch, S. M. (2003). Race differences in depressive symptoms: a dynamic perspective on stress exposure and vulnerability. Journal of Health \& Social Behavior, 44(3), 353-369.

Gershon, E. S., Hamovit, J., Guroff, J. J., Dibble, E., Leckman, J. F., Sceery, W., et al. (1982). A family study of schizoaffective, bipolar I, bipolar II, unipolar, and normal control probands. Archives of General Psychiatry, 39(10), 1157-1167.

Gielen, A. C., McDonnell, K. A., Wu, A. W., O'Campo, P., \& Faden, R. (2001). Quality of life among women living with HIV: the importance violence, social support, and self care behaviors. Social Science \& Medicine, 52(2), 315-322.

Gillock, K. L., Zayfert, C., Hegel, M. T., \& Ferguson, R. J. (2005). Posttraumatic stress disorder in primary care: prevalence and relationships with physical symptoms and medical utilization. General Hospital Psychiatry, 27(6), 392-399.

Ginsburg, G. S., \& Drake, K. L. (2002). School-based treatment for anxious africanamerican adolescents: a controlled pilot study. Journal of the American Academy of Child \& Adolescent Psychiatry, 41(7), 768-775.

Girdler, S. S., Thompson, K. S., Light, K. C., Leserman, J., Pedersen, C. A., Prange, A. J., Jr., et al. (2004). Historical sexual abuse and current thyroid axis profiles in women with premenstrual dysphoric disorder. Psychosomatic Medicine, 66(3), 403-410.

Goodwin, R. D., Kroenke, K., Hoven, C. W., \& Spitzer, R. L. (2003). Major depression, physical illness, and suicidal ideation in primary care. Psychosomatic Medicine, 65(4), 501-505.

Gore, J. L., Krupski, T., Kwan, L., Fink, A., \& Litwin, M. S. (2005). Mental health of low income uninsured men with prostate cancer. Journal of Urology, 173(4), 1323-1326.

Gore, M., Brandenburg, N. A., Dukes, E., Hoffman, D. L., Tai, K. S., Stacey, B., et al. (2005). Pain severity in diabetic peripheral neuropathy is associated with patient functioning, symptom levels of anxiety and depression, and sleep. Journal of Pain \& Symptom Management, 30(4), 374-385.

Graesser, A. C., Cai, Z., Louwerse, M., \& Daniel, F. (2006). Question understanding aid (QUAID): A web facility that helps survey methodologists improve the comprehensibility of questions. Public Opinion Quarterly, 70, 1-20.

Greenberg, M. A., \& Stone, A. A. (1992). Emotional disclosure about traumas and its relation to health: effects of previous disclosure and trauma severity. Journal of Personality \& Social Psychology, 63(1), 75-84. 
Gutman, D. A., \& Nemeroff, C. B. (2003). Persistent central nervous system effects of an adverse early environment: clinical and preclinical studies. Physiology \& Behavior, 79(3), 471-478.

Hamilton, M. (1959). The assessment of anxiety states by rating. British Journal of Medical Psychology, 32(1), 50-55.

Hamilton, M. (1967). Development of a rating scale for primary depressive illness. . British Journal of Sociological and Clinical Psychology, 6(4), 278-296.

Hannah, P., Adams, D., Lee, A., Glover, V., \& Sandler, M. (1992). Links between early post-partum mood and post-natal depression. British Journal of Psychiatry, 160, 777-780.

Harter, M. C., Conway, K. P., \& Merikangas, K. R. (2003). Associations between anxiety disorders and physical illness. European Archives of Psychiatry \& Clinical Neuroscience, 253(6), 313-320.

Harter, S. L. (2000). Psychosocial adjustment of adult children of alcoholics: a review of the recent empirical literature. Clinical Psychology Review, 20(3), 311-337.

Hasin, D. S., Stinson, F. S., Ogburn, E., \& Grant, B. F. (2007). Prevalence, correlates, disability, and comorbidity of DSM-IV alcohol abuse and dependence in the United States: results from the National Epidemiologic Survey on Alcohol and Related Conditions. Archives of General Psychiatry, 64(7), 830-842.

Havlir, D. V., \& Richman, D. D. (1996). Viral dynamics of HIV: implications for drug development and therapeutic strategies. Annals of Internal Medicine, 124(11), 984-994.

Helzer, J. E., \& Pryzbeck, T. R. (1988). The co-occurrence of alcoholism with other psychiatric disorders in the general population and its impact on treatment. Journal of Studies on Alcohol, 49(3), 219-224.

Hensyl, W. R. (Ed.) (1990) Stedman's medical dictionary (25th ed.). Baltimore, Williams \& Wilkins.

Herek, G. M., Capitanio, J. P., \& Widaman, K. F. (2002). HIV-related stigma and knowledge in the United States: prevalence and trends, 1991-1999. American Journal of Public Health, 92(3), 371-377.

Hesselbrock, M. N., Meyer, R. E., \& Keener, J. J. (1985). Psychopathology in hospitalized alcoholics. Archives of General Psychiatry, 42(11), 1050-1055.

Hirschfeld, R. M. (2001). The comorbidity of major depression and anxiety disorders: Recognition and management in primary care. Primary Care Companion to Journal of Clinical Psychiatry, 3(6), 244-254.

Holahan, C. J., Moos, R. H., Holahan, C. K., Cronkite, R. C., \& Randall, P. K. (2003). Drinking to cope and alcohol use and abuse in unipolar depression: a 10-year model. Journal of Abnormal Psychology, 112(1), 159-165.

Holcomb, W. L., Jr., Stone, L. S., Lustman, P. J., Gavard, J. A., \& Mostello, D. J. (1996). Screening for depression in pregnancy: characteristics of the Beck Depression Inventory. Obstetrics \& Gynecology, 88(6), 1021-1025.

Holt, R., Court, P., Vedhara, K., Nott, K. H., Holmes, J., \& Snow, M. H. (1998). The role of disclosure in coping with HIV infection. AIDS Care, 10(1), 49-60.

Hooyman, N. R., \& Gonyea, J. G. (1999). A feminist model of family care: practice and policy directions. Journal of Women \& Aging, 11(2/3), 149-169. 
Hough, E. S., Brumitt, G. A., \& Templin, T. N. (1999). Social support, demands of illness, and depression in chronically ill urban women. Health Care for Women International, 20(4), 349-362.

Howell, E. A., Mora, P., \& Leventhal, H. (2006). Correlates of early postpartum depressive symptoms. Maternal \& Child Health Journal, 10(2), 149-157.

Hudson, A., Kirksey, K., \& Holzemer, W. (2004). The influence of symptoms on quality of life among HIV-infected women. Western Journal of Nursing Research, 26(1), 9-30.

Hulley, S. B., Cummings, S. R., Brower, W. S., Grady, D., Hearst, N., Newman, T. B. (Ed.). (2001). Designing clinical research: An epidemiological approach. Philadelphia: Lippincott, Williams, and Wilkins.

Husaini, B. A., Castor, R. S., Linn, G., Moore, S. T., Warren, H. A., \& Whitten-Stovall, R. (1990). Social support and depression: Among the black and white elderly. Journal of Community Psychiatry, 18, 12-18.

Hutton, H. E., Lyketsos, C. G., Zenilman, J. M., Thompson, R. E., \& Erbelding, E. J. (2004). Depression and HIV risk behaviors among patients in a sexually transmitted disease clinic. American Journal of Psychiatry, 161(5), 912-914.

Huurre, T., Eerola, M., Rahkonen, O., \& Aro, H. (2007). Does social support affect the relationship between socioeconomic status and depression? A longitudinal study from adolescence to adulthood. Journal of Affective Disorders, 100(1-3), 55-64.

Ickovics, J. R., Hamburger, M. E., Vlahov, D., Schoenbaum, E. E., Schuman, P., Boland, R. J., et al. (2001). Mortality, CD4 cell count decline, and depressive symptoms among HIV-seropositive women: longitudinal analysis from the HIV Epidemiology Research Study. JAMA, 285(11), 1466-1474.

Issakidis, C., Sanderson, K., Corry, J., Andrews, G., \& Lapsley, H. (2004). Modelling the population cost-effectiveness of current and evidence-based optimal treatment for anxiety disorders. Psychological Medicine, 34(1), 19-35.

Jenkins, S. R., \& Coons, H. L. (Eds.). (1996 ). Psychological stress and adaptation processes for women coping with HIV/AIDS. . New York: Plenum Press. .

Kahn, K. L., Pearson, M. L., Harrison, E. R., Desmond, K. A., Rogers, W. H., Rubenstein, L. V., et al. (1994). Health care for black and poor hospitalized Medicare patients.[see comment]. JAMA, 271(15), 1169-1174.

Kalichman, S. C., DiMarco, M., Austin, J., Luke, W., \& DiFonzo, K. (2003). Stress, social support, and HIV-status disclosure to family and friends among HIVpositive men and women. Journal of Behavioral Medicine, 26(4), 315-332.

Kalichman, S. C., Rompa, D., \& Cage, M. (2000). Distinguishing between overlapping somatic symptoms of depression and HIV disease in people living with HIVAIDS. Journal of Nervous \& Mental Disease, 188(10), 662-670.

Kendler, K. S., Davis, C. G., \& Kessler, R. C. (1997). The familial aggregation of common psychiatric and substance use disorders in the National Comorbidity Survey: a family history study. British Journal of Psychiatry, 170, 541-548.

Kessler, D., Lloyd, K., Lewis, G., \& Gray, D. P. (1999). Cross sectional study of symptom attribution and recognition of depression and anxiety in primary care. British Medical Journal, 318(7181), 436-439.

Kessler, R. C. (1982). A disaggregation of the relationship between socioeconomic status and psychological distress. American Sociological Review, 47(6), 752-764. 
Kessler, R. C. (2003). Epidemiology of women and depression. Journal of Affective Disorders, 74(1), 5-13.

Kessler, R. C., Berglund, P., Demler, O., Jin, R., Koretz, D., Merikangas, K. R., et al. (2003). The epidemiology of major depressive disorder: results from the National Comorbidity Survey Replication (NCS-R). JAMA, 289 (23), 3095-3105.

Kessler, R. C., Berglund, P., Demler, O., Jin, R., Merikangas, K. R., Walters, E. E., et al. (2005). Lifetime prevalence and age-of-onset distributions of DSM-IV disorders in the National Comorbidity Survey Replication. Archives of General Psychiatry, 62(6), 593-602.

Kessler, R. C., Berglund, P. A., Dewit, D. J., Ustun, T. B., Wang, P. S., Wittchen, H. U., et al. (2002). Distinguishing generalized anxiety disorder from major depression: prevalence and impairment from current pure and comorbid disorders in the US and Ontario. International Journal of Methods in Psychiatric Research, 11(3), 99111.

Kessler, R. C., Crum, R. M., Warner, L. A., Nelson, C. B., Schulenberg, J., \& Anthony, J. C. (1997). Lifetime co-occurrence of DSM-III-R alcohol abuse and dependence with other psychiatric disorders in the National Comorbidity Survey. Archives of General Psychiatry, 54(4), 313-321.

Kessler, R. C., McGonagle, K. A., Nelson, C. B., Hughes, M., Swartz, M., \& Blazer, D. G. (1994). Sex and depression in the National Comorbidity Survey. II: Cohort effects. Journal of Affective Disorders, 30(1), 15-26.

Kessler, R. C., McGonagle, K. A., Zhao, S., Nelson, C. B., Hughes, M., Eshleman, S., et al. (1994). Lifetime and 12-month prevalence of DSM-III-R psychiatric disorders in the United States. Results from the National Comorbidity Survey. Archives of General Psychiatry, 51(1), 8-19.

King, C. A., Knox, M. S., Henninger, N., Nguyen, T. A., Ghaziuddin, N., Maker, A., et al. (2006). Major depressive disorder in adolescents: family psychiatric history predicts severe behavioral disinhibition. Journal of Affective Disorders, 90(2-3), 111-121.

Klein, D. N., Lewinsohn, P. M., Seeley, J. R., \& Rohde, P. (2001). A family study of major depressive disorder in a community sample of adolescents. Archives of General Psychiatry, 58(1), 13-20.

Kosten, T. A., Anton, S. F., \& Rounsaville, B. J. (1992). Ascertaining psychiatric diagnoses with the family history method in a substance abuse population. Journal of Psychiatric Research, 26(2), 135-147.

Kripke, D. F., Jean-Louis, G., Elliott, J. A., Klauber, M. R., Rex, K. M., Tuunainen, A., et al. (2004). Ethnicity, sleep, mood, and illumination in postmenopausal women. BMC Psychiatry, 4, 8.

Kroenke, C. H., Bennett, G. G., Fuchs, C., Giovannucci, E., Kawachi, I., Schernhammer, E., et al. (2005). Depressive symptoms and prospective incidence of colorectal cancer in women. American Journal of Epidemiology, 162(9), 839-848.

Kroenke, K., Spitzer, R. L., Williams, J. B., Monahan, P. O., \& Lowe, B. (2007). Anxiety disorders in primary care: prevalence, impairment, comorbidity, and detection. Annals of Internal Medicine, 146(5), 317-325. 
Kushner, M. G., Sher, K. J., \& Erickson, D. J. (1999). Prospective analysis of the relation between DSM-III anxiety disorders and alcohol use disorders. American Journal of Psychiatry, 156(5), 723-732.

Landgraf, R., \& Wigger, A. (2003). Born to be anxious: neuroendocrine and genetic correlates of trait anxiety in HAB rats. Stress, 6(2), 111-119.

Latkin C. A personal network approach to AIDS prevention: an experimental peer group intervention for street-injecting drug users: the SAFE study. In Needle RH, Coyle SL, Genser SG, Trotter RT, eds. Social Networks, Drug Abuse and HIV Transmission. Rockville, MD: National Institute on Drug Abuse; 1991:181-195.

Lee, K. A., Portillo, C. J., \& Miramontes, H. (2001). The influence of sleep and activity patterns on fatigue in women with HIV/AIDS. Journal of the Association of Nurses in AIDS Care, 12 Suppl, 19-27.

Leibschultz, J., Feinman, G., Sullivan, L., Stein, M., \& Samet, J. (2000). Physical and sexual abuse in women infected with the human immunodeficiency virus: increased illness and health care utilization. Archives of Internal Medicine, 160(11), 1659-1664.

Leserman, J. (2003). HIV disease progression: depression, stress, and possible mechanisms. Biological Psychiatry, 54(3), 295-306.

Leserman, J., Jackson, E. D., Petitto, J. M., Golden, R. N., Silva, S. G., Perkins, D. O., et al. (1999). Progression to AIDS: the effects of stress, depressive symptoms, and social support. Psychosomatic Medicine, 61(3), 397-406.

Lester, D. (Ed.). (2000). Alcoholism, substance abuse, and suicide. New York: Guilford Press.

Lester, R., \& Petrie, T. A. (1998). Physical, psychological, and societal correlates of bulimic symptomatology among African American college women. Journal of Counseling Psychology, 45 315-321

Lieb, R., Isensee, B., Hofler, M., Pfister, H., \& Wittchen, H. U. (2002). Parental major depression and the risk of depression and other mental disorders in offspring: a prospective-longitudinal community study. Archives of General Psychiatry, 59(4), 365-374.

Liebschutz, J. M., Finley, E. P., Braslins, P. G., Christiansen, D., Horton, N. J., \& Samet, J. H. (2003). Screening for sexually transmitted infections in substance abuse treatment programs. Drug \& Alcohol Dependence, 70(1), 93-99.

Link, B. G., Lennon, M. C., \& Dohrenwend, B. P. (1993). Socioeconomic status and depression: The role of occupations involving direction, control, and planning. . American Journal of Sociology 98, 1351-1387.

Lipsitz, J. D., Williams, J. B., Rabkin, J. G., Remien, R. H., Bradbury, M., el Sadr, W., et al. (1994). Psychopathology in male and female intravenous drug users with and without HIV infection. American Journal of Psychiatry, 151(11), 1662-1668.

Lloyd-Williams, M., Friedman, T., \& Rudd, N. (2000). Criterion validation of the Edinburgh postnatal depression scale as a screening tool for depression in patients with advanced metastatic cancer. Journal of Pain \& Symptom Management, 20(4), 259-265.

Lo-Biondo-Wood, G., \& Haber, J. (1994). Nursing research: Methods, critical appraisal, and utilization.. St.Louis: Mosby. 
Loring, M., \& Powell, B. (1988). Gender, race, and DSM-III: a study of the objectivity of psychiatric diagnostic behavior. Journal of Health \& Social Behavior, 29(1), 122.

Lovejoy, N. C. (1990). AIDS: Impact on the gay man's homosexual and heterosexual families Marriage andFamily Review 14 285-316.

Low-Beer, S., Chan, K., Yip, B., Wood, E., Montaner, J. S., O'Shaughnessy, M. V., et al. (2000). Depressive symptoms decline among persons on HIV protease inhibitors. Journal of Acquired Immune Deficiency Syndromes: JAIDS, 23(4), 295-301.

Lowy, M. T., Wittenberg, L., \& Yamamoto, B. K. (1995). Effect of acute stress on hippocampal glutamate levels and spectrin proteolysis in young and aged rats. Journal of Neurochemistry, 65(1), 268-274.

Ludman, E. J., Katon, W., Russo, J., Von Korff, M., Simon, G., Ciechanowski, P., et al. (2004). Depression and diabetes symptom burden. General Hospital Psychiatry, 26(6), 430-436.

Lundqvist, G., Hansson, K., \& Svedin, C. G. (2004). The influence of childhood sexual abuse factors on women's health. Nordic Journal of Psychiatry, 58(5), 395-401.

Lustberg, L., \& Reynolds, C. F. (2000). Depression and insomnia: Questions of cause and effect. . Sleep Medicine Reviews, 4(3), 253-262.

Lyketsos, C. G., Hoover, D. R., Guccione, M., Dew, M. A., Wesch, J. E., Bing, E. G., et al. (1996). Changes in depressive symptoms as AIDS develops. The Multicenter AIDS Cohort Study. American Journal of Psychiatry, 153(11), 1430-1437.

Maier, W., Lichtermann, D., \& Minges, J. (1994). The relationship between alcoholism and unipolar depression--a controlled family study. Journal of Psychiatric Research, 28(3), 303-317.

Maj, M., Janssen, R., Starace, F., Zaudig, M., Satz, P., Sughondhabirom, B., et al. (1994). WHO Neuropsychiatric AIDS study, cross-sectional phase I. Study design and psychiatric findings. Archives of General Psychiatry, 51(1), 39-49.

Mayberry, R. M., Mili, F., \& Ofili, E. (2000). Racial and ethnic differences in access to medical care. Medical Care Research \& Review, 57 Suppl 1, 108-145.

McBarnette, L. S. (Ed.). (1996). African American women Newbury Park: Sage Publications.

McDaniel, J. S., Fowlie, E., Summerville, M. B., Farber, E. W., \& Cohen-Cole, S. A. (1995). An assessment of rates of psychiatric morbidity and functioning in HIV disease. General Hospital Psychiatry, 17(5), 346-352.

McEwen, B. S. (1998). Stress, adaptation, and disease. Allostasis and allostatic load. Annals of the New York Academy of Sciences, 840, 33-44.

McEwen, B. S. (2002). Mood disorders and allostatic load. Biological Psychiatry, 54(3), 200-207.

McEwen, B. S. (2006). Sleep deprivation as a neurobiologic and physiologic stressor: Allostasis and allostatic load. Metabolism: Clinical \& Experimental, 55(10 Suppl 2), S20-23.

McKellar, J. D., Humphreys, K., \& Piette, J. D. (2004). Depression increases diabetes symptoms by complicating patients' self-care adherence. Diabetes Educator, 30(3), 485-492.

McLeod, D. R., Foster, G. V., Hoehn-Saric, R., Svikis, D. S., \& Hipsley, P. A. (1994). Family history of alcoholism in women with generalized anxiety disorder who 
have premenstrual syndrome: patient reports of premenstrual alcohol consumption and symptoms of anxiety. Alcoholism: Clinical \& Experimental Research, 18(3), 664-670.

Merikangas, K. R., Mehta, R. L., Molnar, B. E., Walters, E. E., Swendsen, J. D., AguilarGaziola, S., et al. (1998). Comorbidity of substance use disorders with mood and anxiety disorders: results of the International Consortium in Psychiatric Epidemiology. Addictive Behaviors, 23(6), 893-907.

Metcalfe, K. A., Langstaff, J. E., Evans, S. J., Paterson, H. M., \& Reid, J. L. (1998). Meeting the needs of women living with HIV. Public Health Nursing, 15(1), 3034.

Meynell, J., \& Barroso, J. (2005). Bioimpedance analysis and HIV-related fatigue. JANAC: Journal of the Association of Nurses in AIDS Care, 16(2), 13-22.

Mirochnick, M. (2000). Antiretroviral pharmacology in pregnant women and their newborns. Annals of the New York Academy of Sciences, 918, 287-297.

Mirowsky, J., \& Ross, C. E. (1989). Psychiatric diagnosis as reified measurement. Journal of Health \& Social Behavior, 30(1), 11-25; discussion 26-40.

Mish, F. C. (Ed.) (1997) The Merriam-Webster Dictionary (5th ed., Vols. ). Springfield: Merriam-Webster.

Mock, K. S., Phillips, K. D., \& Sowell, R. L. (2002). Depression and sleep dysfunction as predictors of fatigue in HIV-infected African-American women. Clinical Excellence for Nurse Practitioners, 6(2), 9-16.

Moneyham, L., Seals, B., Demi, A., Sowell, R., Cohen, L., \& Guillory, J. (1996a). Experiences of disclosure in women infected with HIV. Health Care for Women International, 17(3), 209-221.

Moneyham, L., Seals, B., Demi, A., Sowell, R., Cohen, L., \& Guillory, J. (1996b). Perceptions of stigma in women infected with HIV. AIDS Patient Care \& STDs, 10(3), 162-167.

Monroe, S. M., \& Simons, A. D. (1991). Diathesis-stress theories in the context of life stress research: implications for the depressive disorders. Psychological Bulletin, 110(3), 406-425.

Moore, J., Schuman, P., Schoenbaum, E., Boland, B., Solomon, L., \& Smith, D. (1999). Severe adverse life events and depressive symptoms among women with, or at risk for, HIV infection in four cities in the United States of America. AIDS, 13(17), 2459-2468.

Morrison, M. F., Petitto, J. M., Ten Have, T., Gettes, D. R., Chiappini, M. S., Weber, A. L., et al. (2002). Depressive and anxiety disorders in women with HIV infection. American Journal of Psychiatry, 159(5), 789-796.

Mosack, V., \& Shore, E. R. (2003). Screening for depression among pregnant and postpartum women Journal of Community Health Nursing 23(1), 37-47.

Motivala, S. J., Sarfatti, A., Olmos, L., \& Irwin, M. R. (2005). Inflammatory markers and sleep disturbance in major depression. Psychosomatic Medicine, 67(2), 187-194.

Murphy, G. (Ed.). (2000). Psychiatric aspects of suicidal behavior: Substance abuse. . Chichester, UK: John Wiley and Sons.

Murphy, J. M., Horton, N. J., Laird, N. M., Monson, R. R., Sobol, A. M., \& Leighton, A. H. (2004). Anxiety and depression: a 40-year perspective on relationships 
regarding prevalence, distribution, and comorbidity. Acta Psychiatrica Scandinavica, 109(5), 355-375.

Murray, L., Cooper, P. J., \& Stein, A. (1991). Postnatal depression and infant development.. British Medical Journal, 302(6783), 978-979.

Murrell, S. A., Himmelfarb, S., \& Wright, K. (1983). Prevalence of depression and its correlates in older adults. American Journal of Epidemiology, 117(2), 173-185.

Myers, J. K., \& Weissman, M. M. (1980). Use of a self-report symptom scale to detect depression in a community sample. American Journal of Psychiatry137, 10811084.

Myers, J. K., Weissman, M. M., Tischler, G. L., Holzer, C. E., 3rd, Leaf, P. J., Orvaschel, H., et al. (1984). Six-month prevalence of psychiatric disorders in three communities 1980 to 1982. Archives of General Psychiatry, 41(10), 959-967.

National Institute of Mental Health. (n/d). National Institute of Mental Health. Retrieved 4-7-08, from http://www.nimh.nih.gov/

National Institutes of Health. (1979). The Belmont Report: Ethical principles and guidelines for the protection of human subjects of research. Retrieved 4-16-08, from http://www.nih.training.com/ohsite/guidelines/belmont.html,

Neighbors, H. W., Jackson, J. S., Campbell, L., \& Williams, D. (1989). The influence of racial factors on psychiatric diagnosis: a review and suggestions for research. Community Mental Health Journal, 25(4), 301-311.

Neighbors, H. W., Trierweiler, S. J., Ford, B. C., \& Muroff, J. R. (2003). Racial differences in DSM diagnosis using a semi-structured instrument: the importance of clinical judgment in the diagnosis of African Americans. Journal of Health \& Social Behavior, 44(3), 237-256.

Neubauer, D. N. (2004). Chronic insomnia: current issues. Clinical Cornerstone, 6 Suppl $1 C, \mathrm{~S} 17-22$.

Niccolai, L. M., Dorst, D., Myers, L., \& Kissinger, P. J. (1999). Disclosure of HIV status to sexual partners: predictors and temporal patterns. Sexually Transmitted Diseases, 26(5), 281-285.

Nokes, K. M., Chidekel, J. H., \& Kendrew, J. (1999). Exploring the complexity of sleep disturbances in persons with HIV/AIDS. JANAC: Journal of the Association of Nurses in AIDS Care, 10(3), 22-29.

Nokes, K. M., \& Kendrew, J. (1996). Sleep quality in people with HIV disease. J Assoc Nurses in AIDS Care 7(3), 43-50.

Nokes, K. M., \& Kendrew, J. (2001). Correlates of sleep quality in persons with HIV disease. JANAC: Journal of the Association of Nurses in AIDS Care, 12(1), 17-22.

Norman, S. E., Chediak, A. D., Freeman, C., Kiel, M., Mendez, A., Duncan, R., et al. (1992). Sleep disturbances in men with asymptomatic human immunodeficiency (HIV) infection. Sleep, 15(2), 150-155.

Nott, P. N., Franklin, M., Armitage, C., \& Gelder, M. G. (1976). Hormonal changes and mood in the puerperium. British Journal of Psychiatry, 128, 379-383.

O'Hara, M. W., \& Gorman, L. L. (2004). Can postpartum depression be predicted? Primary Psychiatry: The Leading Voice of Clinical Psychiatric Medicine, 11(3), 42. 
Olfson, M., \& Pincus, H. A. (1996). Outpatient mental health care in nonhospital settings: distribution of patients across provider groups. American Journal of Psychiatry, 153(10), 1353-1356.

Olfson, M., Shea, S., Feder, A., Fuentes, M., Nomura, Y., Gameroff, M., et al. (2000). Prevalence of anxiety, depression, and substance use disorders in an urban general medicine practice. Archives of Family Medicine, 9(9), 876-883.

Peleikis, D. E., Mykletun, A., \& Dahl, A. A. (2004). The relative influence of childhood sexual abuse and other family background risk factors on adult adversities in female outpatients treated for anxiety disorders and depression. Child Abuse \& Neglect, 28(1), 61-76.

Penhazur, E. J., \& Schmelkin, L. P. (1991). Measurement, design, and analysis: An integrated approach. . Hilsdale: Lawrence Erlbaum Associates.

Pennebaker, J. W., Colder, M., \& Sharp, L. K. (1990). Accelerating the coping process. Journal of Personality \& Social Psychology, 58(3), 528-537.

Penzak, S. R., Reddy, Y. S., \& Grimsley, S. R. (2000). Depression in patients with HIV infection. American Journal of Health-System Pharmacy, 57(4), 376-386; quiz 387-379.

Perkins, D. O., Stern, R. A., Golden, R. N., Murphy, C., Naftolowitz, D., \& Evans, D. L. (1994). Mood disorders in HIV infection: prevalence and risk factors in a nonepicenter of the AIDS epidemic. American Journal of Psychiatry, 151(2), 233-236.

Phillips, K. D., Moneyham, L., Murdaugh, C., Boyd, M. R., Tavakoli, A., Jackson, K., et al. (2005). Sleep disturbance and depression as barriers to adherence. Clinical Nursing Research, 14(3), 273-293.

Plant, E. A., \& Sachs-Ericsson, N. (2004). Racial and ethnic differences in depression: the roles of social support and meeting basic needs. Journal of Consulting \& Clinical Psychology, 72(1), 41-52.

Plat, L., Leproult, R., L'Hermite-Baleriaux, F., Mockel, J., Polonsky, K. S., \& Van Carter, E. (1999). Metabolic effects of short-term elevations of plasma cortisol are more pronounced in the evening than in the morning. . Journal of Clinicaland Endocrinogyl Metabolism, 84, 3082-3092.

Polit, D. F., \& Hungler, B. P. (1995). Nursing research principles and methods (5th ed.). Philadelphia: Lippincott

Rabkin, J. G., Goetz, R. R., Remien, R. H., Williams, J. B., Todak, G., \& Gorman, J. M. (1997). Stability of mood despite HIV illness progression in a group of homosexual men. American Journal of Psychiatry, 154(2), 231-238.

Rabkin, J. G., Johnson, J., Lin, S. H., Lipsitz, J. D., Remien, R. H., Williams, J. B., et al. (1997). Psychopathology in male and female HIV-positive and negative injecting drug users: longitudinal course over 3 years. AIDS, 11(4), 507-515.

Rabkin, J. G., Wagner, G. J., \& Rabkin, R. (1999). Fluoxetine treatment for depression in patients with HIV and AIDS: a randomized, placebo-controlled trial. American Journal of Psychiatry, 156(1), 101-107.

Radloff, L. S. (1977). The CES-D scale: A self-report depression scale for researching the general population. Applied Psychological Measurement, 1, 385-401. 
Raskin, A., Schulterbrandt, J., Reatig, N., \& McKeon, J. J. (1969). Replication of factors of psychopathology in interview, ward behavior and self-report ratings of hospitalized depressives. Journal of Nervous \& Mental Disease, 148(1), 87-98.

Reardon, S. F., \& Buka, S. L. (2002). Differences in onset and persistence of substance abuse and dependence among whites, blacks, and Hispanics. Public Health Reports, 117 Suppl 1, S51-59.

Regier, D. A., Boyd, J. H., Burke, J. D., Jr., Rae, D. S., Myers, J. K., Kramer, M., et al. (1988). One-month prevalence of mental disorders in the United States. Based on five Epidemiologic Catchment Area sites. Archives of General Psychiatry, 45(11), 977-986.

Regier, D. A., Farmer, M. E., Rae, D. S., Locke, B. Z., Keith, S. J., Judd, L. L., et al. (1990). Comorbidity of mental disorders with alcohol and other drug abuse. Results from the Epidemiologic Catchment Area (ECA) Study. JAMA, 264(19), 2511-2518.

Reich, T., Cloninger, C. R., Van Eerdwegh, P., Rice, J. P., \& Mullaney, J. (1988). Secular trends in the familial transmission of alcoholism. Alcoholism: Clinical and Experimental Research, 12, 458-464.

Reif, S., Whetten, K., Ostermann, J., \& Raper, J. L. (2006). Characteristics of HIVinfected adults in the Deep South and their utilization of mental health services: A rural vs. urban comparison. AIDS Care, 18 Suppl 1, S10-17.

Reiger, D. A., Farmer, M. E., \& Rae, D. S. (1993). One-month prevalence of mental disoders in the United States and sociodemographic characteristics: the epidemiologic catchment area study. . Acta Psychiatr Scand, 88, 35-47.

Reinherz, H. Z., Paradis, A. D., Giaconia, R. M., Stashwick, C. K., \& Fitzmaurice, G. (2003). Childhood and adolescent predictors of major depression in the transition to adulthood. American Journal of Psychiatry, 160(12), 2141-2147.

Rice, J. P., Neuman, R. J., Saccone, N. L., Corbett, J., Rochberg, N., Hesselbrock, V., et al. (2003). Age and birth cohort effects on rates of alcohol dependence. Alcoholism: Clinical \& Experimental Research, 27(1), 93-99.

Ringdahl, E. N., Pereira, S. L., \& Delzell, J. E., Jr. (2004). Treatment of primary insomnia. Journal of the American Board of Family Practice, 17(3), 212-219.

Robbins, J. L., Phillips, K. D., Dudgeon, W. D., \& Hand, G. A. (2004). Physiological and psychological correlates of sleep in HIV infection. Clinical Nursing Research, 13(1), 33-52.

Rodriguez, B. G., Weisberg, R. G., Pagano, M. E., Machan, J. T., Culpepper, L., \& Keller, M. B. (2004). Frequency and patterns of psychiatric comorbidity in a sample of primary care patients with anxiety disorders Comprehensive Psychiatry, 45, 129-137.

Roper, W. L. (1991). Current approaches to prevention of HIV infections. Public Health Reports, 106(2), 111-115.

Ross, C. E., \& Mirowsky, J. (1989). Explaining the social patterns of depression: control and problem solving--or support and talking? Journal of Health \& Social Behavior, 30(2), 206-219.

Ross, L. E., Gilbert Evans, S. E., Sellers, E. M., \& Romach, M. K. (2003). Measurement issues in postpartum depression part 1: anxiety as a feature of postpartum depression. Archives of Women's Mental Health, 6(1), 51-57. 
Rubinstein, M. L., \& Selwyn, P. A. (1998). High prevalence of insomnia in an outpatient population with HIV infection. JAIDS and Human Retrovirology 19, 260-265.

Rudisch, B., \& Nemeroff, C. B. (2003). Epidemiology of comorbid coronary artery disease and depression. Biological Psychiatry, 54(3), 227-240.

Ruo, B., Rumsfeld, J. S., Hlatky, M. A., Liu, H., Browner, W. S., Whooley, M. A., et al. (2003). Depressive symptoms and health-related quality of life: the Heart and Soul Study. JAMA, 290(2), 215-221.

Rutter, M., Silberg, J., O'Connor, T., \& Simonoff, E. (1999a). Genetics and child psychiatry: I Advances in quantitative and molecular genetics. Journal of Child Psychology \& Psychiatry \& Allied Disciplines, 40(1), 3-18.

Rutter, M., Silberg, J., O'Connor, T., \& Simonoff, E. (1999b). Genetics and child psychiatry: II Empirical research findings. Journal of Child Psychology \& Psychiatry \& Allied Disciplines, 40(1), 19-55.

Sacco, W. P., \& Beck, A. T. (1995). Cognitive theory and therapy. New York: Guilford.

Sagrestano, L. M., Paikoff, R. L., Holmbeck, G. N., \& Fendrich, M. (2003). A longitudinal examination of familial risk factors for depression among inner-city African American adolescents. Journal of Family Psychology, 17(1), 108-120.

Sandelowski, M., Barroso, J., Sandelowski, M., \& Barroso, J. (2003). Motherhood in the context of maternal HIV infection. Research in Nursing \& Health, 26(6), 470482.

Sartorius, N., Ustun, T. B., Lecrubier, Y., \& Wittchen, H. U. (1996). Depression comorbid with anxiety: results from the WHO study on psychological disorders in primary health care. British Journal of Psychiatry - Supplementum(30), 38-43.

Schmidt, C. K., \& Goggin, K. (2002). Disclosure patterns among HIV-positive women. American Clinical Laboratory, 40-43.

Schrimshaw, E. W. (2003). Relationship-specific unsupportive social interactions and depressive symptoms among women living with HIV/AIDS: direct and moderating effects. Journal of Behavioral Medicine, 26(4), 297-313.

Schroeder, M. A. (1990). Diagnosing and dealing with multicollinearity. Western Journal of Nursing Research, 12(2), 175-187.

Schutte, K. K., Moos, R. H., \& Brennan, P. L. (1995). Depression and drinking behavior among women and men: a three-wave longitudinal study of older adults. Journal of Consulting \& Clinical Psychology, 63(5), 810-822.

Selye, H. (1974). Stress without distress. . Toronto: McClelland and Stewart.

Shahar, G., \& Davidson, L. (2003). Depressive symptoms erode self-esteem in severe mental illness: a three-wave, cross-lagged study. Journal of Consulting \& Clinical Psychology, 71(5), 890-900.

Sharp, L. K., \& Lipsky, M. S. (2002). Screening for depression across the lifespan: a review of measures for use in primary care settings. American Family Physician, 66(6), 1001-1008.

Sher, L. (2004). Daily hassles, cortisol, and the pathogenesis of depression. Medical Hypotheses, 62(2), 198-202.

Shiner, R. L., \& Marmorstein, N. R. (1998). Family environments of adolescents with lifetime depression: Associations with maternal depression history. Journal of the American Academy of Child and Adolescent Psychiatry, 37, 1152-1160. 
Simon, G. E., Goldberg, D. P., Von Korff, M., \& Ustun, T. B. (2002). Understanding cross-national differences in depression prevalence. Psychological Medicine, 32(4), 585-594.

Smedley, B. D. (2006). Expanding the frame of understanding health disparities: from a focus on health systems to social and economic systems. Health Education \& Behavior, 33(4), 538-541.

Smith, D. K., Moore, J. S., Warren, D., Solomon, L., Schuman, P., Stein, M., et al. (Eds.). (1996). The design, participants, and selected early findings of the HIV Epidemiology Research (HER) Study. . New York: Plenum Press.

Smith, M. T., Perlis, M. L., Smith, M. S., Giles, D. E., \& Carmody, T. P. (2000). Sleep quality and presleep arousal in chronic pain. Journal of Behavioral Medicine, 23(1), 1-13.

Smith, S. M., Stinson, F. S., Dawson, D. A., Goldstein, R., Huang, B., Grant, B. F., et al. (2006). Race/ethnic differences in the prevalence and co-occurrence of substance use disorders and independent mood and anxiety disorders: Results from the National Epidemiologic Survey on Alcohol and Related Conditions. Psychological Medicine, 36(7), 987-998.

Snowden, L. R., \& Thomas, K. (2000). Medicaid and African American outpatient mental health treatment. . Mental Health Service Research, 2, 115-120.

Sobell, L. C., \& Sobell, M. B. (1978). Validity of self-reports in three populations of alcoholics. Journal of Consulting \& Clinical Psychology, 46(5), 901-907.

Starace, F., Ammassari, A., Trotta, M. P., Murri, R., De Longis, P., Izzo, C., et al. (2002). Depression is a risk factor for suboptimal adherence to highly active antiretroviral therapy. Journal of Acquired Immune Deficiency Syndromes: JAIDS, 31 Suppl 3, S136-139.

Stein, M. D., Freedberg, K. A., Sullivan, L. M., Savetsky, J., Levenson, S. M., Hingson, R., et al. (1998). Sexual ethics: Disclosure of HIV-positive status to partners. Arch Intern Med, 158(3), 253-257. Arch Intern Med, 158(3), 253-257.

Stein, M. D., \& Samet, J. H. (1999). Disclosure of HIV status. AIDS Patient Care \& STDs, 13(5), 265-267.

Sterling, P., \& Eyer, J. (Eds.). (1998). Allostasis: a new paradigm to explain arousal pathology. . New York: John Wiley.

Stern, R. A., Singer, N. G., Silva, S. G., Rogers, H. J., Perkins, D. O., Hall, C. D., et al. (1992). Neurobehavioral functioning in a nonconfounded group of asymptomatic HIV-seropositive homosexual men. American Journal of Psychiatry, 149(8), 1099-1102.

Sullivan, P. F., Wells, J. E., Joyce, P. R., Bushnell, J. A., Mulder, R. T., \& OakleyBrowne, M. A. (1996). Family history of depression in clinic and community samples. Journal of Affective Disorders, 40(3), 159-168.

Sussman, L. K., Robins, L. N., \& Earls, F. (1987). Treatment-seeking for depression by black and white Americans. Social Science \& Medicine, 24(3), 187-196.

Swain, A. M., O'Hara, M. W., Starr, K. R., \& Gorman, L. L. (1997). A prospective study of sleep, mood, and cognitive function in postpartum and nonpostpartum women. Obstetrics \& Gynecology, 90(3), 381-386.

Tam, T., Weisner, C., \& Mertens, J. (2000). Demographic characteristics ,life context, and patterns of substance abuse among alcohol-dependent treatment clients in a 
health maintenance organization. . Alcoholism: Clinical Experimental Research, 24(12), 1803-1810.

Taylor, E. R., Amodei, N., \& Mangos, R. (1996). The presence of psychiatric disorders in HIV-infected women. Journal of Counseling Development, 74, 345-351.

Teresi, J. A., Abrams, R., Holmes, D., Ramirez, M., Shapiro, C., Eimicke, J. P., et al. (2002). Influence of cognitive impairment, illness, gender, and African-American status on psychiatric ratings and staff recognition of depression. American Journal of Geriatric Psychiatry, 10(5), 506-514.

Todd, R. D., Geller, B., Neuman, R., Fox, L. W., \& Hickok, J. (1996). Increased prevalence of alcoholism in relatives of depressed and bipolar children. Journal of the American Academy Child and Adolescent Psychiatry, 35(6), 716-724.

Tsao, J. C., Dobalian, A., Moreau, C., \& Dobalian, K. (2004). Stability of anxiety and depression in a national sample of adults with human immunodeficiency virus. Journal of Nervous \& Mental Disease, 192(2), 111-118.

Turner, R. J., \& Lloyd, D. A. (1999). The stress process and the social distribution of depression. Journal of Health \& Social Behavior, 40(4), 374-404.

Turrina, C., Fiorazzo, A., Turano, A., Cacciani, P., Regini, C., Castelli, F., et al. (2001). Depressive disorders and personality variables in HIV-positive and negative intravenous drug-users. Journal of Affective Disorders, 65(1), 45-53

U. S. Census Bureau. (2006). American Community Survey. Retrieved 4-16-08, from http://www.census.gov/population/www/socdemo/educ-attn.html

UNAIDS. (2000, 12-7-2000). Trends in HIV prevalence: natural course of the epidemic or results of behavioural change? Retrieved 4-16-08, from http://data.unaids.org/Publications/IRC-pub04/una99-12_trends-hivincidence_en.pdf

United States Department of Health and Human Services. (1999). Mental Health: A report of the Surgeon General. . Rockville, MD.: National Institutes of Health.

United States Preventive Services Task Force. (2002). Screening for depression: recommendations and rationale. Annals of Internal Medicine, 136, 760-764.

USDHHS. (1999). Mental Health: A report of the Surgeon General. Retrieved. from.

USDHHS Health Resources and Services Administration, H. A. B. (2002). The AIDS epidemic and the Ryan White care act: Past progress, future challenges. Retrieved 2-12-07, from http://www.hab.hrsa.gov

Valdiserri, R. O. (2002). HIV/AIDS stigma: an impediment to public health.[comment]. American Journal of Public Health, 92(3), 341-342.

Valente, S. M. (2003). Depression and HIV disease. Journal of the Association of Nurses in AIDS Care, 14(2), 41-51.

Valente, S. M., \& Saunders, J. M. (1997). Managing depression among people with HIV disease. Journal of the Association of Nurses in AIDS Care, 8(1), 51-67.

van Heeringen, K. (2003). The neurobiology of suicide and suicidality. Canadian Journal of Psychiatry - Revue Canadienne de Psychiatrie, 48(5), 292-300.

Vanable, P. A., Carey, M. P., Blair, D. C., \& Littlewood, R. A. (2006). Impact of HIVrelated stigma on health behaviors and psychological adjustment among HIVpositive men and women. AIDS \& Behavior, 10(5), 473-482. 
Von Korff, M., \& Simon, G. (1996). The prevalence and impact of psychological disorders in primary care: HMO research needed to improve care. HMO Practice, 10(4), 150-155.

Vyavaharkar, M., Moneyham, L., Tavakoli, A., Phillips, K. D., Murdaugh, C., Jackson, K., et al. (2007). Social support, coping, and medication adherence among HIVpositive women with depression living in rural areas of the southeastern United States. AIDS Patient Care \& STDs, 21(9), 667-680.

Wallsten, S. S. (2000). Effects of caregiving, gender, and race on the health, mutuality, and social supports of older couples. Journal of Aging \& Health, 12(1), 90-111.

Walrath, C., Ybarra, M., Holden, E. W., Liao, Q., Santiago, R., Leaf, P., et al. (2003). Children with reported histories of sexual abuse: utilizing multiple perspectives to understand clinical and psychosocial profiles. Child Abuse \& Neglect, 27(5), 509524.

Wang, P. S., Lane, M., Olfson, M., Pincus, H. A., Wells, K. B., \& Kessler, R. C. (2005). Twelve-Month Use of Mental Health Services in the United States: Results From the National Comorbidity Survey Replication. Archives of General Psychiatry, 62(6), 629-640.

Weissman, M. M., Gammon, G. D., John, K., Merikangas, K. R., Warner, V., Prusoff, B. A., et al. (1987). Children of depressed parents: Increased psychopathology and early onset of major depression. Archives of General Psychiatry, 44, 847-853.

Weissman, M. M., Warner, V., Wickamaratne, P., Moreau, D., \& Olfson, M. (1997). Offspring of depressed patients: 10 years later. Archives of General Psychiatry, (54), 932-940.

Weissman, M. M., Wickramaratne, P., Merikangas, K. R., Leckman, J. F., Prustoff, B. A., Caruso, K. A., et al. (1984). Onset of major depression in early adulthood: Increased familial loading and specificity. Archives of General Psychiatry, 41, 1136-1143.

Weissman, M. M., Wickramaratne, P., Nomura, Y., Warner, V., Verdeli, H., Pilowsky, D. J., et al. (2005). Families at high and low risk for depression: a 3-generation study. Archives of General Psychiatry, 62(1), 29-36.

Welch, K. J. (2000). Correlates of alcohol and/or drug use among HIV-infected individuals. AIDS Patient Care, 14(6), 317-323.

Wells, K., Klap, R., Koike, A., \& Sherbourne, C. (2001). Ethnic disparities in unmet need for alcoholism, drug abuse, and mental health care. American Journal of Psychiatry, 158(12), 2027-2032.

Wells, K. B., Golding, J. M., \& Burnam, M. A. (1989). Affective, substance use, and anxiety disorders in persons with arthritis, diabetes, heart disease, high blood pressure, or chronic lung conditions. General Hospital Psychiatry, 11(5), 320327.

Wells, K. B., Stewart, A., Hays, R. D., Burnam, M. A., Rogers, W., Daniels, M., et al. (1989). The functioning and well-being of depressed patients. Results from the Medical Outcomes Study. JAMA, 262(7), 914-919.

Wilhelm, K., \& Parker, G. (1994). Sex differences in lifetime depression rates: fact or artefact? Psychological Medicine, 24(1), 97-111. 
Williams, S., \& Dale, J. (2006). The effectiveness of treatment for depression/depressive symptoms in adults with cancer: a systematic review. British Journal of Cancer, 94(3), 372-390.

Williamson, D. E., Birmaher, B., Axelson, D. A., Ryan, N. D., \& Dahl, R. E. (2004). First episode of depression in children at low and high familial risk for depression. Journal of the American Academy of Child \& Adolescent Psychiatry, 43(3), 291-297.

Wilsnack, R. W., Klassen, A. D., \& Wilsnack, S. C. (1986). Retrospective analysis of lifetime changes in women's drinking behavior. Advances in Alcohol \& Substance Abuse, 5(3), 9-28.

Winokur, G., \& Coryell, W. (1991). Familial alcoholism in primary unipolar major depressive disorder. American Journal of Psychiatry, 148(2), 184-188.

Wittchen, H. U. (2002). Generalized anxiety disorder: prevalence, burden, and cost to society. Depression \& Anxiety, 16(4), 162-171.

Wittchen, H. U., Kessler, R. C., Beesdo, K., Krause, P., Hofler, M., Hoyer, J., et al. (2002). Generalized anxiety and depression in primary care: prevalence, recognition, and management. Journal of Clinical Psychiatry, 63 Suppl 8, 24-34.

Wohl, A. R., Lu, S., Odem, S., Sorvillo, F., Pegues, C. F., \& Kerndt, P. R. (1998). Sociodemographic and behavioral characteristics of Africam American women with HIV And AIDS in Los Angeles County, 1990-1997 JAIDS 19(4), 413-420.

World Health Organization. (1990). Composite International Diagnostic Interview (CIDI, Version 1). Geneva: WHO.

World Health Organization. (1992). Basic documents (Vol. 39th). Geneva: WHO.

World Health Organization. (2004). Mental Health. Retrieved 4-7-08, 2008, from http://www.who.int/mental_health/en/

Zea, M. C., Reisen, C. A., Poppen, P. J., Bianchi, F. T., \& Echeverry, J. J. (2005). Disclosure of HIV status and psychological well-being among Latino gay and bisexual men. AIDS \& Behavior, 9(1), 15-26.

Zhang, A. Y., \& Snowden, L. R. (1999). Ethnic characteristics of mental disorders in five U.S. communities. Cultural Diversity \& Ethnic Minority Psychology, 5(2), 134146.

Zimmerman, M. M. D., \& Chelminski, I. P. D. (2003). Generalized Anxiety Disorder in Patients With Major Depression: Is DSM-IV's Hierarchy Correct? American Journal of Psychiatry, 160(3), 504-512.

Zung, W. W. (1965). A self-rating depression scale. Archives of General Psychiatry, 12, 63-70. 
APPENDICES 


\section{APPENDIX A. DEMOGRAPHIC QUESTIONNAIRE}

Predictors of Anxiety and Depressive Symptoms

Nina $\mathrm{K}$. Sublette, $\mathrm{PhD}$ candidate, $\mathrm{RN}$; FNP

Preparation date: May 31, 2006

Page 1 of 4

\section{Demographic Questionnaire}

\section{Participant Identification \#}

Please answer each question as honestly as you can.

1. Age (fill in the blank)

2. Marital Status: (Circle One)

Single

Married

Divorced

Widowed

Living with a Partner in the same household, but not married

3. Do you have any children?

(Circle One) yes no

If yes, how many?

4. What is the highest grade you have completed? (Circle One)

$7^{\text {th }}-9^{\text {th }}$ grade

$10^{\text {th }}-11^{\text {th }}$

High School Graduate or GED

Some college or vocational training

College Graduate

Master's Degree or higher

5. Yearly income (Circle One)

Less than $\$ 5000$ per year

$\$ 5000-\$ 10,000$

$\$ 10,000-\$ 15,000$

$\$ 15,000-\$ 20,000$

$\$ 20,000-\$ 40,000$

$\$ 40,000$ or higher

6. Do you have a job at this time? (Circle one)

Yes, full- time

Yes, part-time

No, I am unemployed

No, but I am looking for a job

7. How long have you been diagnosed as HIV-positive? 


\section{Predictors of Anxiety and Depressive Symptoms}

Nina $\mathrm{K}$. Sublette, $\mathrm{PhD}$ candidate, RN, FNP

Preparation date: May 31, 2006

Page 2 of 4

8. Who have you told that you were HIV-positive? (Circle all that apply)

No one

Current partner

Previous partner

Friend

Mother

Father

Grandparent

Brother or Sister

Other family member

Minister or church member

Other

The next four questions ask about the way you have been feeling over the last 2 weeks.

9. Over the past two weeks, have you felt down, depressed, or hopeless?

$$
\text { (Circle one) yes }
$$

no

10. Over the past two weeks, have you felt little interest or pleasure in doing things?

(Circle one) yes

no

11. Over the past two weeks, have you felt anxious or worried?

(Circle one) yes

no

12. Alcohol or drug use in the past two weeks?

(Circle one) yes

no

If yes, what kind? (Circle all that apply)

Beer
Liquor (mixed/ frozen drink)
Cocaine
Crystal Meth
Tobacco

Beer

Cocaine

Tobacco

\author{
Wine or Wine Cooler \\ Marijuana \\ Crack Cocaine \\ Heroin
}




\section{Predictors of Anxiety and Depressive Symptoms}

Nina $\mathrm{K}$. Sublette, $\mathrm{PhD}$ candidate, $\mathrm{RN}$, FNP

Preparation date: May 31, 2006

Page 3 of 4

The next two questions ask about your mental health history.

13. Have you ever been diagnosed with depression, anxiety, or any other mood disorder?

$$
\text { (Circle one) yes no }
$$

14. Have you ever been hospitalized for depression, anxiety or any other mood disorder?

$$
\text { (Circle one) yes no }
$$

The next two questions are about your family history.

15. Do you have a family history of drug or alcohol abuse?

$$
\text { (Circle one) yes no }
$$

16. Do you have a family history of mood disorders? (For example: Nerve problems, anxiety, mental illness, bipolar, depression)

$$
\text { (Circle One) yes no }
$$

The next questions ask about the things that you do that may affect your sleep.

17. What time do you usually go to bed? $\mathrm{AM}$ or $\mathrm{PM}$

18. What things do you usually do before you go to sleep (Circle all that apply)

Brush teeth

Read

Bath or Shower

other (please describe)
Put on pajamas

Eat

Watch TV

19. What time do you usually get up?

20. Do you take naps?

$$
\text { (Circle one) yes no }
$$

If yes, how long do the naps last?

How many naps a day?

21. Do you take medicine that helps you sleep?

(Circle one) yes no

22. Do you usually sleep alone?

(Circle one) yes no
If no, who usually sleeps with you?


Predictors of Anxiety and Depressive Symptoms

Nina $\mathrm{K}$. Sublette, $\mathrm{PhD}$ candidate, $\mathrm{RN}$, FNP

Preparation date: May 31, 2006

Page 4 of 4

23. Where do you usually sleep?

(Circle one): $\quad$ Bed $\quad$ Couch Floor

The next questions ask about your sexual history.

24. How old were you when you started having periods or cycles?

25. How old were you the first time you had sex (vaginal intercourse)?

26. How old was your partner?

27. Did you want to have sex with this partner?

(Circle One) yes no

If no, were you forced?

(Circle One) yes no

28 . Have you ever been sexually assaulted or raped?

(Circle One) yes no

If yes, How many times?

How old were you?

Did you tell anyone about your sexual assault?

(Circle One) yes no

Were you under the influence of drugs or alcohol at the time of the sexual assault?

(Circle One) yes

no

29. Have you ever had a sexually transmitted infection other than HIV?

(Circle One) yes no

30. How many sex partners have you had? (In your lifetime?)

31. Did you use condoms the last time you had sex?

(Circle One) yes no

32. Did you use any drugs or alcohol during or immediately before your last sexual experience?

(Circle one) yes

no 


\section{APPENDIX B. PITTSBURGH SLEEP QUALITY INDEX (PSQI)}

\section{Pittsburgh Sleep Quality Index (PSQI)}

Instructions: The following questions relate to your usual sleep habits during the past month only. Your answers should indicate the most accurate reply for the majority of days and nights in the past month. Please answer all questions.

During the past month,

1. When have you usually gone to bed?

2. How long (in minutes) has it taken you to fall asleep each night?

3. When have you usually gotten up in the morning?

4. How many hours of actual sleep did you get that night? (This may be different than the number of hours you spend in bed)

\begin{tabular}{|l|l|l|l|l|}
\hline $\begin{array}{l}\text { 5. During the past month, how often have you had } \\
\text { trouble sleeping because you... }\end{array}$ & $\begin{array}{c}\text { Not during } \\
\text { the past } \\
\text { month (0) }\end{array}$ & $\begin{array}{c}\text { Less than } \\
\text { once a } \\
\text { week (1) }\end{array}$ & $\begin{array}{c}\text { Once or } \\
\text { twice a } \\
\text { week (2) }\end{array}$ & $\begin{array}{c}\text { Three or } \\
\text { more times } \\
\text { a week (3) }\end{array}$ \\
\hline a. Cannot get to sleep within 30 minutes & & & & \\
\hline b. Wake up in the middle of the night or early morning & & & & \\
\hline c. Have to get up to use the bathroom & & & & \\
\hline d. Cannot breathe comfortably & & & & \\
\hline e. Cough or snore loudly & & & & \\
\hline f. Feel too cold & & & & \\
\hline $\begin{array}{l}\text { g. Feel too hot } \\
\text { h. Have bad dreams }\end{array}$ & & & & \\
\hline i. Have pain & & & & \\
\hline $\begin{array}{l}\text { j. Other reason(s), please describe, including how often } \\
\text { you have had trouble sleeping because of this } \\
\text { reason(s): }\end{array}$ & & & & \\
\hline $\begin{array}{l}\text { 6. During the past month, how often have you taken } \\
\text { medicine (prescribed or "over the counter") to help you } \\
\text { sleep? }\end{array}$ & & Fairly & $\begin{array}{c}\text { Fairly } \\
\text { bad (2) }\end{array}$ & bad (3) \\
\hline $\begin{array}{l}\text { 7. During the past month, how often have you had } \\
\text { trouble staying awake while driving, eating meals, or } \\
\text { engaging in social activity? }\end{array}$ & & & & \\
\hline $\begin{array}{l}\text { 8. During the past month, how much of a problem has it } \\
\text { been for you to keep up enthusiasm to get things } \\
\text { done? }\end{array}$ & & & & \\
\hline $\begin{array}{l}\text { 9. During the past month, how would you rate your sleep } \\
\text { quality overall? }\end{array}$ & & & & \\
\hline
\end{tabular}

Component 1 \#9 Score

Component 2 \#2 Score ( $\leq 15 \mathrm{~min}(0), 16-30 \mathrm{~min}(1), 31-60 \mathrm{~min}(2),>60 \mathrm{~min}(3))$

$+\# 5$ a Score (if sum is equal $0=0 ; 1-2=1 ; 3-4=2 ; 5-6=3$ )

Component 3 \#4 Score $(>7(0), 6-7(1), 5-6(2),<5(3)$

Component 4 (total \# of hours asleep)/(total \# of hours in bed) $\times 100$

$>85 \%=0,75 \%-84 \%=1,65 \%-74 \%=2,<65 \%=3$

Component 5 \# sum of scores $5 b$ to $5 j(0=0 ; 1-9=1 ; 10-18=2 ; 19-27=3)$

Component 6 \#6 Score

Component $7 \quad \# 7$ score $+\# 8$ score $(0=0 ; 1-2=1 ; 3-4=2 ; 5-6=3)$

$\mathrm{C} 1$

Add the seven component scores together Global PSQI Score

Reprinted from Journal of Psychiatric Research, 28(2), Buysse, D.J., Reynolds III, C.F., Monk, T.H., Berman, S.R., \&

Kupfer, D.J. The Pittsburgh Sleep Quality Index: A New Instrument for Psychiatric Practice and Research, 193-213,

Copyright 1989, with permission from Elsevier Science.

Try This: Best Practices in Nursing Care to Older Adults, A series from the Hartford Institute for Geriatric Nursing www.hartfordign.org 


\section{APPENDIX C. CENTER FOR EPIDEMIOLOGIC STUDIES DEPRESSION SCALE (CES-D), NIMH}

\section{Center for Epidemiologic Studies Depression Scale (CES-D), NIMH}

Below is a list of the ways you might have felt or behaved. Please tell me how often you have felt this way during the past week.

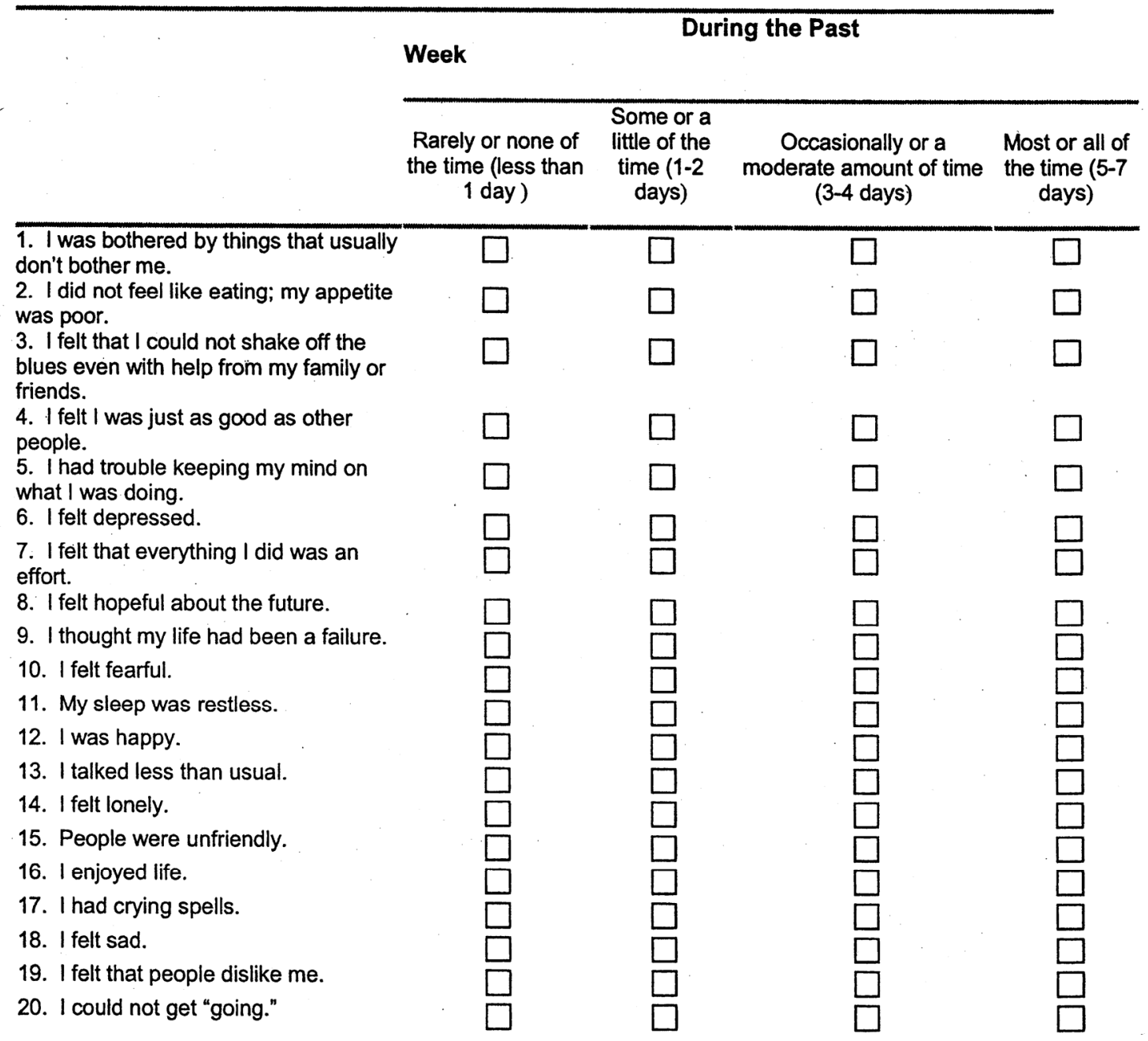

SCORING: zero for answers in the first column, 1 for answers in the second column, 2 for answers in the third column, 3 for answers in the fourth column. The scoring of positive items is reversed. Possible rangc of scores is zero to 60 , with the higher scores indicating the presence of more symptomatology. 


\title{
APPENDIX D. EDINBURGH POSTNATAL DEPRESSION SCALE (EPDS)
}

\author{
Name: \\ Date: \\ Address: \\ Baby's Age:
}

As you have recently had a baby, we would like to know how you are feeling. Please UNDERLINE the answer which comes closest to how you have felt IN THE PAST 7 DAYS, not just how you feel today.

Here is an example, already completed.

I have felt happy:

Yes, all the time

Yes, most of the time

No, not very often

No, not at all

This would mean: "I have felt happy most of the time" during the past week. Please complete the other questions in the same way.

In the past 7 days:

1. I have been able to laugh and see the funny side of things

As much as I always could

Not quite so much now

Definitely not so much now

Not at all

2. I have looked forward with enjoyment to things As much as I ever did

Rather less than I used to

Definitely less than I used to

Hardly at all

*3. .I have blamed myself unnecessarily when things went wrong

Yes, most of the time

Yes, some of the time

Not very often

No, never

4. I have been anxious or worried for no good reason

No, not at all

Hardly ever

Yes, sometimes

Yes, very often

*5. I have felt scared or panicky for no very good reason

Yes, quite a lot

Yes, sometimes

No, not much

No, not at all
*6. Things have been getting on top of me Yes, most of the time I haven't been able to cope at all

Yes, sometimes I haven't been coping as well as usual

No, most of the time I have coped quite well No, have been coping as well as ever

*7. I have been so unhappy that I have had difficulty sleeping

Yes, most of the time

Yes, sometimes

Not very often

No, not at all

*8. I have felt sad or miserable

Yes, most of the time

Yes, quite often

Not very often

No, not at all

*9 I have been so unhappy that I have been crying

Yes, most of the time

Yes, quite often

Only occasionally

No, never

*10.The thought of harming myself has occurred to me Yes, quite often

Sometimes

Hardly ever

Never 


\section{APPENDIX E. INSTITUTIONAL REVIEW BOARD APPROVAL LETTER}

THE UNIVERSITY OF TENNESSEE

Health Science Center

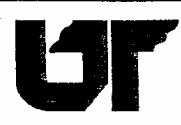

July 17,2006

Institutional Review Board

956 Court Avenue, Suite F208

Memphis, TN 38163

Nina Sublette, APRN, BC

College of Graduate Health Sciences

Department of Nursing

E102 Rout

UTHSC

CAMPUS

RE: "Predictors of Anxiety and Depression Symptoms Among African American HIV-Positive Women" (IRB \#8530)

Dear Ms. Sublette,

We are in receipt of your written acceptance dated July 10, 2006 in response to the provisos outlined in my letter dated June 20, 2006 concerning the above referenced Institutional Review Board Project.

The Administrative Section of the UTHSC Institutional Review Board (IRB) determined your application to be consistent with the guidelines for expedited review under categories (5) and (7). Therefore, this application was approved in this regard as complying with proper consideration of the rights and welfare of human subjects, the risk involved, and the potential benefits of the study.

This letter constitutes full approval of your application, questionnaire(s) and consent form, stamped approved by the UTHSC IRB on July 17, 2006 for the above referenced study. This project was approved for 12 months with an expiration date of June 19, 2007. The consent form dated July 17, 2006 is also approved from the date of this letter through June 19, 2007.

In the event that volunteers, either subjects or patients, are to be recruited by means other than usual and standard patient care practices, the Board must approve of any such solicitation materials (i.e., advertising copies or posters, etc.).

Any alterations (revisions) in the protocol must be promptly reported to and approved by the UTHSC IRB prior to implementation of these revisions. You have individual responsibility for reporting to the Board in the event of unanticipated or serious adverse events and subject deaths.

In addition, reapproval of your project is required by the IRB, and it is the responsibility of the Principal Investigator to initiate the request for continuation (reapproval) regardless of the time the activity has been approved by the sponsoring agency. Your research project may not continue beyond the schedule as indicated above unless you obtain written approval to continue the project.

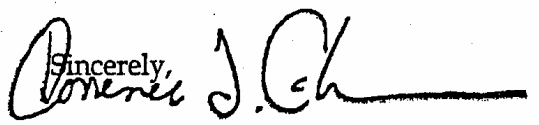

Terrence F. Ackerman, Ph.D.

Executive Vice Chairman

Institutional Review Board 


\title{
APPENDIX F. ROBERT YATES M.D. LETTER OF RECOMMENDATION
}

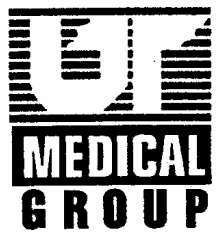

\author{
UT Medical Group, Inc. \\ Obstetrics \& Gynecology \\ 880 Madison Avenue, Sulte 3EOI \\ Memphis, Tennessee 38103-3409 \\ $901-448-6632$ - Fax: $901-448-7352$
}

April 7, 2006

Ms. Nina Sublette, RN, FNP

$\mathrm{PhD}$ Candidate

University of Tennessee

College of Graduate Health Sciences

Memphis, TN 38103

Dear Ms Sublette:

I am very pleased to write this letter in support of the research project The Predictors of Anxiety and Depressive Symptoms among HIV-Positive Women. As the medical director of the UTMG Ob-Gyn clinic, I have worked with this population for many years. Many patients come into the clinic with multiple issues that interfere with their ability to maintain their health. This includes transportation, drug abuse, mental illness, and many other barriers to health that I know must be addressed to assure proper medical care.

The research project that you have proposed will provide screening services that are vital to the health of these women and I welcome a chance for such a project in our clinic. I look forward to working you in this process.

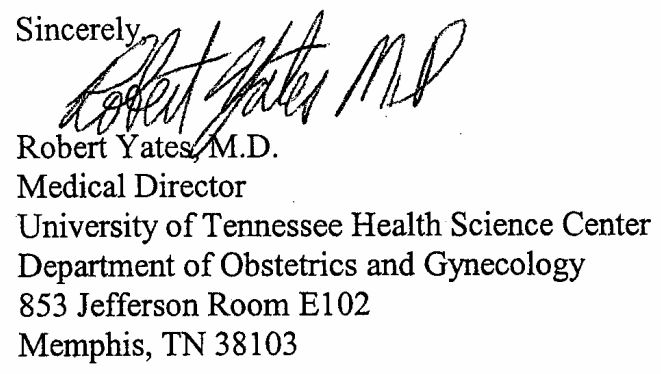

Gynecology - Matemal Fetal Medicine - Obstetrics - Reproductive Genelics Center for Urinary Control

853 Jefferson Avenue. E102 Memphis, TN 38103 $901-448-5771$
880 Madison Avenue Memphis, TN 38103 $901-448-6632$
7945 Wolf River Boulevard Germantown, IN 38138 $901-347-8320$ 


\section{APPENDIX G. MELISSA APPLETON, M. D. LETTER OF RECOMMENDATION}

\begin{tabular}{|c|c|}
\hline $\begin{array}{l}\text { THE UNIVERSITY OF TENNESSEE } \\
\text { Health Science Center }\end{array}$ & \\
\hline & $\begin{array}{r}\text { College of Medicine } \\
\text { Department of Medicine } \\
\text { Division of Infectious Diseases } \\
1211 \text { Union Avenue, Suite } 340 \\
\text { Memphis, TN } 38104 \\
\text { Tel: (901) 448-5770 } \text { Fax: (901) 448-5940 }\end{array}$ \\
\hline
\end{tabular}

May 3, 2006

Ms. Nina Sublette, RN, FNP

$\mathrm{PhD}$ Candidate

University of Tennessee

College of Graduate Health Sciences

Memphis, TN 38103

Dear Ms Sublette:

I am very pleased to write this letter in support of the research project The Predictors of Anxiety and Depressive Symptoms among HIV-Positive Women. As the medical director of the Adult Special Care Clinic, I have worked with this population for many years.

Many patients come into the clinic with multiple issues that interfere with their ability to maintain their health. This includes transportation, drug abuse, mental illness, and many other barriers to health that I know must be addressed to assure proper medical care.

The research project that you have proposed will provide screening services that are vital to the health of these women and I welcome a chance for such a project in our clinic. I look forward to working you in this process.

Sincerely,

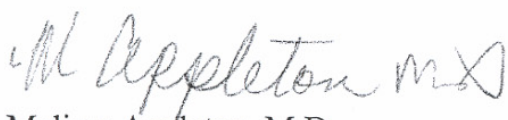

Melissa Appleton, M.D.

Medical Director, Adult Special Care Clinic

The Regional Medical center at Memphis

880 Madison Avenue, $5^{\text {th }}$ Floor

Memphis, TN 38103 


\title{
APPENDIX H. CONSENT FORM
}

\author{
Nina Sublette PI \\ Predictors of Anxiety and Depression \\ Page 1 of 4
}

\section{Consent Form}

Research Protocol:

Principal Investigator:

Co-Investigators:
Predictors of Anxiety and Depressive symptoms in African American HIV-positive Women

Nina $\mathrm{K}$. Sublette, $\mathrm{PhD}$ candidate, $\mathrm{RN}$ FNP

853 Jefferson Ave., Room E102

Memphis, TN 38103

901.448.1347

Mona N. Wicks, PhD, RN

Patricia D. Cunningham, DNSc

\section{Introduction}

You are being given the opportunity to participate in a research study related to depressive and anxiety symptoms in African American HIV-positive women. African American women are the fastest growing group of newly diagnosed HIV cases in the United States. Many HIV-positive persons have symptoms of anxiety and depression during the course of their disease. Symptoms of anxiety and depression could cause the affected person to miss their doctor's appointments. These symptoms may also cause the affected person to not take their medicines as prescribed. Women often have a greater chance of developing anxiety and depression than men. People with chronic health problems like HIV also have a greater risk for developing anxiety and depression. For these reasons we would like to study symptoms of anxiety and depression in African American women who are HIV-positive. We would also like to study the factors that increase the risk for symptoms of anxiety and depression.

The purpose of this study is to determine which risk factors increase the chances for having symptoms of anxiety and depression in African American women who are HIVpositive. This information may help us to know which women should be screened for these symptoms so that they can receive early treatment to manage these symptoms.

A total of 80 women will be enrolled in the study over a period of 3 months. The study will include 20 pregnant and 60 non-pregnant women. The study will be conducted through the University of Tennessee Medical Group's Obstetrics and Gynecology clinic and the Regional Medical Center's Adult Special Care Clinic. Nurses at the University of Tennessee Health Science Center, College of Nursing are conducting this research study. Participation in this study will require only one clinic visit. It will take approximately 20 minutes to answer the questionnaires.

\section{PROCEDURES TO BE FOLLOWED:}

If you agree to participate in this study, you will be asked to read and sign a copy of this informed consent. You will be asked to complete 5 questionnaires. If your scores on the symptoms of depression questionnaire indicate that you need referral for additional 
Nina Sublette PI

Predictors of Anxiety and Depression

Page 2 of 4

screening for these symptoms, a referral will be made to one of the social workers in the clinic.

Demographic information (such as your age and highest education level) and health information (such as the medications you are taking) will be collected as well. We will also review your medical records for health information (such as your lowest and most recent CD4 count).

\section{RISKS ASSOCIATED WITH PARTICIPATION:}

There are no foreseeable physical, social, or economic risks associated with participation in this study. An increase in emotional distress may be caused by thinking about issues related to your personal or family history of some health problems such as drug and alcohol use. You can be referred to the clinic social worker if needed.

\section{BENEFITS ASSOCIATED WITH PARTICIPATION}

You may not receive any health benefits from participating in this study. Your participation will potentially provide valuable information about the underlying risk factors that you have for symptoms of anxiety and depressions. Results from this study may aid in the development of strategies for identifying at risk patients and preventing anxiety and depression. This may help improve health outcomes in HIV-positive persons.

\section{ALTERNATIVES TO PARTICIPATION:}

You may choose not to participate in this study. An alternative to participation in this study is to simply receive the usual screening for symptoms of anxiety and depression that is part of routine health care.

\section{CONFIDENTIALITY:}

You have the right to privacy and all information obtained in this study that can be identified with you will remain confidential within state and federal law. Your research record will be labeled with a code number. A master key that links your name and the code number will be maintained in a separate and secure location. The principal investigator will have access to the information linking questionnaires to personal identifiers. Information contained on the questionnaires will not be placed in the medical record and therefore, access to this information will be denied to other third parties such as employers or insurers. Your identity will not be disclosed in any presentations or publications based on the results of the research study.

Under federal privacy regulations, you have the right to determine who has access to your personal health information (called "protected health information" or PHI). PHI collected in this study may include your medical history, the results of physical exams, lab tests, $x$ ray exams, and other diagnostic and treatment procedures, as well as basic demographic information. By signing this consent form, you are authorizing the researchers at the 
University of Tennessee to have access to your PHI collected in this study and to receive your PHI from your physician and/or facilities where you have received health care. In addition, your PHI may be shared with other persons involved in the conduct or oversight of this research, including researchers at the University of Tennessee College Of Nursing. The Institutional Review Board (IRB) at the University of Tennessee Health Science Center may review your PHI as part of its responsibility to protect the rights and welfare of research subjects. Your PHI will not be used or disclosed to any other person or entity, except as required by law, or for authorized oversight of this research study by other regulatory agencies, or for other research for which the use and disclosure of your PHI has been approved by the IRB. Your PHI will be used only for the research purposes described in the Introduction of this consent form. Your PHI will be used until the study is completed.

You may cancel this authorization in writing at any time by contacting the principal investigator listed on the first page of the consent form. If you cancel the authorization, continued use of your PHI is permitted if it was obtained before the cancellation and its use is necessary in completing the research. However, PHI collected after your cancellation may not be used in the study. If you refuse to provide this authorization, you will not be able to participate in the research study. If you cancel the authorization, then you will be withdrawn from the study. Finally, the federal regulations allow you to obtain access to your PHI collected or used in this study.

\section{COMPENSATION AND TREATMENT FOR INJURY:}

Every reasonable precaution will be taken to ensure my safety during the course of this study. In the event that this research activity results in an injury, treatment will be available, including first aid emergency treatment and follow-up care as needed. Payment for any such treatment must be provided by me or my third party payor, if any, such as health insurance or Medicare. I understand that I am not waiving any legal rights or releasing the University of Tennessee, the Regional Medical Center at Memphis, or the agents of either from liability for negligence. I understand that, in the event of physical injury resulting from the research procedures neither the University of Tennessee nor the Regional Medical Center at Memphis has funds budgeted for compensation either for lost wages or for medical treatment. Therefore, neither the University of Tennessee nor the Regional Medical Center at Memphis provides treatment or reimbursements for such injuries.

\section{QUESTIONS:}

If you have any questions about this study, you can call Nina Sublette 24 hours a day, 7 days a week, at (901) 448-1374 or (901) 533-7700. If you have any questions regarding your rights as a research subject, you may contact Dr. Clair E. Cox, Chairman, Üniversity of Tennessee Institutional Review Board at (901) 448-4824.

\section{PAYMENT FOR PARTICIPATION:}

There is no payment for participating in this study. 
Nina Sublette PI

Predictors of Anxiety and Depression

Page 4 of 4

\section{COSTS OF PARTICIPATION:}

There will be no additional costs to you for participating in this study.

\section{PREMATURE WITHDRAWAL:}

Subjects may discontinue their involvement at anytime by having their questionnaires and related data destroyed. This can be accomplished by contacting the principal investigator, Nina Sublette, at 901-448-1374.

\section{VOLUNTARY PARTICIPATION:}

This study is voluntary, meaning you do not have to participate if you do not want to. You may also voluntarily withdraw from the study after you have agreed to participate. You understand that your refusal to participate or decision to withdraw from the study will involve no penalty or loss of benefits to which you are otherwise entitled.

\section{CONSENT OF SUBJECT:}

I have read or have had read to me the description of the research study as outlined above. The investigator or her representative has explained the study to me and has answered all of the questions I have at this time. I have been told of the potential risks, discomforts, side effects and adverse reactions as well as the possible benefits (if any) of this study.

I freely volunteer to participate in the study. I understand that I do not have to take part in this study and that my refusal to participate will involve no penalty or loss of rights to which I am entitled. I further understand that I am free to later withdraw my consent and discontinue participation in this study at any time. I understand that refusing to participate or later withdrawing from the study will not adversely affect my subsequent medical care. I will be given a copy of the signed consent form for my records.

Signature of Research Subject Date

Signature of Witness Date

Signature of Person Obtaining Consent Date




\section{VITA}

Nina Sublette was born May 25, 1966 in Jackson, TN. She earned a BS in Early Childhood and Elementary Education from The University of Tennessee at Martin in 1989, and a MEd in Counseling and Personnel Services from the University of Memphis in 1990. In 1991, she received a BS in Nursing from The University of Tennessee Health Science Center. She was accepted into the Family Nurse practitioner Program at the Mississippi University of Women in Columbus, Mississippi; she graduated with a MSN degree in 1999. Nina was accepted into the graduate nursing program at The University of Tennessee Health Science Center in 2002 and will graduate from The University of Tennessee with a Ph.D. in Nursing in May, 2008. 